\author{
UNIVERSIDADE DE SÃO PAULO \\ INSTITUTO DE FÍSICA DE SÃO CARLOS
}

Rafael Besse

Estudo das propriedades estruturais, energéticas, eletrônicas e ópticas dos calcogenetos quaternários $\mathbf{A}_{2} \mathbf{M}^{\mathrm{II}} \mathbf{M}_{3}^{\mathrm{IV}} \mathbf{Q}_{8}$

São Carlos 

Rafael Besse

\section{Estudo das propriedades estruturais, energéticas, eletrônicas e ópticas dos calcogenetos quaternários $\mathbf{A}_{2} \mathbf{M}^{\mathrm{II}} \mathbf{M}_{3}^{\mathrm{IV}} \mathbf{Q}_{8}$}

Dissertação apresentada ao Programa de PósGraduação em Física do Instituto de Física de São Carlos da Universidade de São Paulo, para obtenção do título de Mestre em Ciências.

Área de concentração: Física Básica

Orientador: Prof. Dr. Juarez Lopes Ferreira da Silva

\section{Versão corrigida}

(Versão original disponível na Unidade que aloja o Programa)

\section{São Carlos}


AUTORIZO A REPRODUÇÃO E DIVULGAÇÃO TOTAL OU PARCIAL DESTE TRABALHO, POR QUALQÜER MEIO CONVENCIONAL OU ELETRÔNICO PARA FINS DE ESTUDO E PESQUISA, DESDE QUE CITADA A FONTE.

Ficha catalográfica revisada pelo Serviço de Biblioteca e Informação do IFSC, com os dados fornecidos pelo(a) autor(a)

Besse, Rafael

Estudo das propriedades estruturais, energéticas, eletrônicas e ópticas dos calcogenetos quaternários A2MIIMIV3Q8 / Rafael Besse; orientador Juarez Lopes Ferreira da Silva - versão corrigida -- São Carlos, 2016 . $85 \mathrm{p}$.

Dissertação (Mestrado - Programa de Pós-Graduação em Física Básica) -- Instituto de Física de São Carlos, Universidade de São Paulo, 2016.

1. Calcogenetos. 2. Materiais em camadas. 3. Teoria do funcional da densidade. I. Silva, Juarez Lopes Ferreira da, orient. II. Título. 


\section{AGRADECIMENTOS}

Agradeço ao meu orientador, Prof. Dr. Juarez L. F. da Silva, pelo empenho e solicitude durante todo o desenvolvimento do mestrado. Sou muito grato pelo auxílio e direcionamento fornecido nesses anos.

Agradeço aos colegas do Grupo de Teoria Quântica de Nanomateriais pela convivência, colaboração e pelas contribuições na realização desse trabalho.

Agradeço pela infraestrutura proporcionada pelo Instituto de Física de São Carlos e pelo Instituto de Química de São Carlos, e também pelo Centro de Tecnologia da Informação de São Carlos. Agradeço à Fundação de Amparo à Pesquisa do Estado de São Paulo (FAPESP) pela bolsa e apoio financeiro (processo 2014/25924-3), e ao CNPq pelos 2 meses de bolsa no início do mestrado.

Agradeço aos meus pais, Sandra e Luiz Carlos, por toda a ajuda e incentivo. Obrigado por sempre fazerem muito por mim. 



\section{RESUMO}

BESSE, R. Estudo das propriedades estruturais, energéticas, eletrônicas e ópticas dos calcogenetos quaternários $\mathbf{A}_{2} \mathbf{M}^{\mathrm{II}} \mathbf{M}_{3}^{\mathrm{IV}} \mathbf{Q}_{8}$. 2016. 85p. Dissertação (Mestrado em Ciências) - Instituto de Física de São Carlos, Universidade de São Paulo, São Carlos, 2017.

Os calcogenetos têm atraído atenção devido à variedade de propriedades físicas e químicas que exibem, apontando para sua utilização em muitas aplicações tecnológicas, incluindo a possibilidade de se obter novos materiais bidimensionais. Os calcogenetos quaternários $\mathrm{A}_{2} \mathrm{M}^{\mathrm{II}} \mathrm{M}_{3}^{\mathrm{IV}} \mathrm{Q}_{8}$, onde $\mathrm{A}=\mathrm{K}, \mathrm{Cs} ; \mathrm{M}^{\mathrm{II}}=\mathrm{Mg}, \mathrm{Zn}, \mathrm{Cd}, \mathrm{Hg} ; \mathrm{M}^{\mathrm{IV}}=\mathrm{Ge}, \mathrm{Sn} ; \mathrm{Q}=\mathrm{S}, \mathrm{Se}, \mathrm{Te}$, possuem uma grande variabilidade de band gaps e portanto eles podem ser estudados para engenharia de band gap através de mudanças na composição química. Além disso, dois tipos de estruturas cristalinas são observados nessa família, um formado por empilhamento de camadas, e outro definido por uma rede tridimensional fechada. Assim, é importante entender os fatores que afetam a estabilidade de estruturas em camadas desses compostos complexos. Nesse trabalho, os materiais $\mathrm{A}_{2} \mathrm{M}^{\mathrm{II}} \mathrm{M}_{3}^{\mathrm{IV}} \mathrm{Q}_{8}$ são estudados com cálculos de teoria do funcional da densidade, usando funcionais de troca e correlação semi-local e híbrido, e correções de van der Waals. Os parâmetros de rede variam com a composição conforme o esperado com base no raio atômico. A redução do número atômico de um dos componentes, principalmente $\mathrm{Q}$, aumenta a energia de coesão, devido à intensificação das interações iônicas. Os resultados de energia de ligação entre camadas demonstram a importância das interações de van der Waals, e os valores são similares aos reportados na literatura para vários materiais. Seguindo a tendência de funcionais semi-locais, os band gaps são subestimados, mas cálculos com o funcional híbrido fornecem valores mais apropriados. Os resultados mostram a diversidade de band gaps e uma correlação aproximadamente linear entre band gap e volume da célula unitária. O band gap é principalmente afetado pela mudança do calcogênio, em que o aumento do número atômico diminui o band gap, devido ao aumento da energia dos estados $p$ de Q. As análises dos coeficientes de absorção óptica e elementos de matriz de transição mostram que não existe diferença significativa entre band gap fundamental e óptico nesses materiais. O estudo de estabilidade relativa das estruturas em 9 compostos, com diferentes A e Q, mostra que os raios atômicos têm um importante papel. A estrutura sem formação de camadas é favorecida comparada com as estruturas em camadas apenas na região de raios intermediários, o que é explicado com base na diminuição das tensões na estrutura e em interações coulombianas entre íons da rede.

Palavras-chave: Calcogenetos. Materiais em camadas. Teoria do funcional da densidade. 



\section{ABSTRACT}

BESSE, R. Study of the structural, energetic, electronic and optical properties of quaternary chalcogenides $\mathbf{A}_{2} \mathbf{M}^{\mathbf{I I}} \mathbf{M}_{3}^{\mathbf{I V}} \mathbf{Q}_{8}$. 2016. 85p. Dissertação (Mestrado em Ciências) - Instituto de Física de São Carlos, Universidade de São Paulo, São Carlos, 2017.

Chalcogenides have attracted attention due to the variety of physical and chemical properties which they display, pointing to their use in many technological applications, including the possibility to obtain new bidimensional materials. The quaternary chalcogenides $\mathrm{A}_{2} \mathrm{M}^{\mathrm{II}} \mathrm{M}_{3}^{\mathrm{IV}} \mathrm{Q}_{8}$, where $\mathrm{A}=\mathrm{K}, \mathrm{Cs} ; \mathrm{M}^{\mathrm{II}}=\mathrm{Mg}, \mathrm{Zn}, \mathrm{Cd}, \mathrm{Hg} ; \mathrm{M}^{\mathrm{IV}}=\mathrm{Ge}, \mathrm{Sn} ; \mathrm{Q}=\mathrm{S}$, Se, Te, have a large variability of band gaps and therefore they can be studied for band gap engineering through changes in the chemical composition. Futhermore, two types of crystal structure are observed in this family, one formed by the stacking of layers, and the other defined by a closed three dimensional framework. Thus, it is important to understand the factors that affect the stability of layered structures of these complex compounds. Here, the materials $\mathrm{A}_{2} \mathrm{M}^{\mathrm{II}} \mathrm{M}_{3}^{\mathrm{IV}} \mathrm{Q}_{8}$ are studied with density functional theory calculations, using semi-local and hybrid exchange-correlation functionals, and van der Waals corrections. Lattice parameters vary with composition according to expected based on the atomic radius. The reduction of the atomic number of one of the components, mainly $Q$, increases the cohesive energy, due to the intensification of the ionic interactions. The results of interlayer binding energies demonstrate the importance of van der Waals interactions, and the values are simillar to those reported in the literature for several materials. Following the trend of semi-local functionals, band gaps are underestimated, but hybrid functional calculations provide more accurate values. The results show the diversity of band gaps and an approximate linear correlation between band gap and unit cell volume. The band gap is mainly affected by changing the chalcogen, in which the increase of the atomic number decreases the band gap, due to the increase in the energy of $\mathrm{Q} p$ states. The analysis of optical absorption coefficients and transition matrix elements show that there is no significative difference between fundamental and optical band gap in these materials. The study of relative stability of the structures in 9 compounds, with different A and Q, shows that the atomic radii have an important role. The structure without layer formation is favored compared with the layered structures only in the region of intermediate radii, which is explained based on the reduction of strain in the structure and coulomb interactions between ions in the framework.

Keywords: Chalcogenides. Layered materials. Density functional theory. 



\section{LISTA DE FIGURAS}

Figura 1 - Testes de convergência para a estrutura triclínica de $\mathrm{K}_{2} \mathrm{ZnSn}_{3} \mathrm{~S}_{8}$ em cálculos realizados com PBE+D3. (a) Energia por átomo da estrutura em função da energia de corte. (b) Volume da célula unitária em função da energia de corte. (c) Energia por átomo da estrutura em função do número de pontos $\mathbf{k}$ irredutíveis. (d) Volume da célula unitária em função do número de pontos k irredutíveis. . . . . . . . . . . . . . . . . 37

Figura 2 - Testes de convergência para a estrutura cúbica de $\mathrm{K}_{2} \mathrm{ZnSn}_{3} \mathrm{~S}_{8}$ em cálculos realizados com PBE+D3. (a) Energia por átomo da estrutura em função da energia de corte. (b) Volume da célula unitária em função da energia de corte. (c) Energia por átomo da estrutura em função do número de pontos $\mathbf{k}$ irredutíveis. (d) Volume da célula unitária em função do número de pontos $\mathbf{k}$ irredutíveis. . . . . . . . . . . . . . . . . 38

Figura 3 - Representação das estruturas cristalinas ortorrômbica e monoclínica dos compostos $\mathrm{Cs}_{2} \mathrm{M}^{\mathrm{II}} \mathrm{M}_{3}^{\mathrm{IV}} \mathrm{Q}_{8}$. Os átomos de Cs, $\mathrm{M}^{\mathrm{II}}$, $\mathrm{M}^{\mathrm{IV}}$, Q são representados por esferas de cor ciano, laranja, roxo e verde, respectivamente. 40

Figura 4 - Parâmetros de rede de equilíbrio, $a_{0}$, $b_{0}$ e $c_{0}$, da estrutura ortorrômbica dos 24 compostos $\mathrm{Cs}_{2} \mathrm{M}^{\mathrm{II}} \mathrm{M}_{3}^{\mathrm{IV}} \mathrm{Q}_{8}\left(\mathrm{M}^{\mathrm{II}}=\mathrm{Mg}\right.$, Zn, Cd, Hg; $\mathrm{M}^{\mathrm{IV}}=\mathrm{Ge}$, $\mathrm{Sn} ; \mathrm{Q}=\mathrm{S}, \mathrm{Se}, \mathrm{Te})$. Os gráficos da primeira linha correspondem aos resultados obtidos com o funcional $\mathrm{PBE}$, e os da segunda linha foram obtidos com PBE+TS+SCS.

Figura 5 - Energia de coesão dos 24 compostos $\mathrm{Cs}_{2} \mathrm{M}^{\mathrm{II}} \mathrm{M}_{3}^{\mathrm{IV}} \mathrm{Q}_{8}\left(\mathrm{M}^{\mathrm{II}}=\mathrm{Mg}, \mathrm{Zn}, \mathrm{Cd}\right.$, $\mathrm{Hg} ; \mathrm{M}^{\mathrm{IV}}=\mathrm{Ge}, \mathrm{Sn} ; \mathrm{Q}=\mathrm{S}$, Se, Te), obtidas com PBE e PBE+TS+SCS. 45

Figura 6 - Cargas efetivas médias de cada espécie química nos materiais $\mathrm{Cs}_{2} \mathrm{M}^{\mathrm{II}} \mathrm{M}_{3}^{\mathrm{IV}} \mathrm{Q}_{8}$ $\left(\mathrm{M}^{\mathrm{II}}=\mathrm{Mg}, \mathrm{Zn}, \mathrm{Cd}, \mathrm{Hg} ; \mathrm{M}^{\mathrm{IV}}=\mathrm{Ge}, \mathrm{Sn} ; \mathrm{Q}=\mathrm{S}\right.$, Se, Te). Os resultados da linha superior correspondem aos cálculos com PBE, e os da linha inferior foram obtidos com PBE+TS+SCS. . . . . . . . . . . .

Figura 7 - Energias de ligação entre camadas dos 24 compostos $\mathrm{Cs}_{2} \mathrm{M}^{\mathrm{II}} \mathrm{M}_{3}^{\mathrm{IV}} \mathrm{Q}_{8}\left(\mathrm{M}^{\mathrm{II}}\right.$ $\left.=\mathrm{Mg}, \mathrm{Zn}, \mathrm{Cd}, \mathrm{Hg} ; \mathrm{M}^{\mathrm{IV}}=\mathrm{Ge}, \mathrm{Sn} ; \mathrm{Q}=\mathrm{S}, \mathrm{Se}, \mathrm{Te}\right)$, obtidas com PBE e $\mathrm{PBE}+\mathrm{TS}+\mathrm{SCS} \ldots \ldots \ldots \ldots \ldots \ldots$

Figura 8 - Primeira Zona de Brillouin da rede ortorrômbica. Os pontos destacados e o caminho indicado correspondem às linhas usadas para o cálculo das estruturas de bandas dos compostos $\mathrm{Cs}_{2} \mathrm{M}^{\mathrm{II}} \mathrm{M}_{3}^{\mathrm{IV}} \mathrm{Q}_{8}$. . . . . . . . . . .

Figura 9 - Estruturas de bandas dos compostos $\mathrm{Cs}_{2} \mathrm{CdGe}_{3} \mathrm{Q}_{8}(\mathrm{Q}=\mathrm{S}, \mathrm{Se}, \mathrm{Te})$, calculadas com PBE. O topo da banda de valência está deslocado para energia zero. . . . . . . . . . . . . . . . . . . 50 50 
Figura 10 - Estruturas de bandas dos compostos $\mathrm{Cs}_{2} \mathrm{ZnSn}_{3} \mathrm{Q}_{8}(\mathrm{Q}=\mathrm{S}, \mathrm{Se}, \mathrm{Te})$, calculadas com PBE. O topo da banda de valência está deslocado para energia zero. . . . . . . . . . . . . . . . . 5 51

Figura 11 - Densidades de estados por célula cristalina dos compostos $\mathrm{Cs}_{2} \mathrm{CdGe}_{3} \mathrm{Q}_{8}$ $(\mathrm{Q}=\mathrm{S}, \mathrm{Se}, \mathrm{Te})$, calculadas com PBE. O topo da banda de valência está deslocado para energia zero. . . . . . . . . . . . . . . . 52

Figura 12 - Densidades de estados por célula cristalina dos compostos $\mathrm{Cs}_{2} \mathrm{ZnSn}_{3} \mathrm{Q}_{8}$ $(\mathrm{Q}=\mathrm{S}$, Se, Te), calculadas com PBE. O topo da banda de valência está deslocado para energia zero. . . . . . . . . . . . . . . . . 53

Figura 13 - Comparação entre as DOS do composto $\mathrm{Cs}_{2} \mathrm{CdGe}_{3} \mathrm{~S}_{8}$ obtidas com PBE e com PBE+TS+SCS. O topo da banda de valência foi colocado na energia zero. . . . . . . . . . . . . . . . . . 5 54

Figura 14 - Valores de band gap no ponto $\Gamma$ em função do volume da célula unitária para os 24 calcogenetos $\mathrm{Cs}_{2} \mathrm{M}^{\mathrm{II}} \mathrm{M}_{3}^{\mathrm{IV}} \mathrm{Q}_{8}\left(\mathrm{M}^{\mathrm{II}}=\mathrm{Mg}, \mathrm{Zn}, \mathrm{Cd}, \mathrm{Hg} ; \mathrm{M}^{\mathrm{IV}}=\right.$ $\mathrm{Ge}, \mathrm{Sn} ; \mathrm{Q}=\mathrm{S}, \mathrm{Se}, \mathrm{Te})$. Os resultados mostrados na primeira coluna correspondem às estruturas de equilíbrio de $\mathrm{PBE}$, enquanto a segunda coluna contém os resultados das estruturas de PBE+TS+SCS. Os resultados da linha superior foram obtidos com o funcional PBE, enquanto os da segunda linha foram obtidos com HSE06.

Figura 15 - Coeficientes de absorção óptica dos 24 calcogenetos $\mathrm{Cs}_{2} \mathrm{M}^{\mathrm{II}} \mathrm{M}_{3}^{\mathrm{IV}} \mathrm{Q}_{8}\left(\mathrm{M}^{\mathrm{II}}\right.$ $=\mathrm{Mg}, \mathrm{Zn}, \mathrm{Cd}, \mathrm{Hg} ; \mathrm{M}^{\mathrm{IV}}=\mathrm{Ge}, \mathrm{Sn} ; \mathrm{Q}=\mathrm{S}$, Se, Te) obtidos com PBE. As curvas mostradas são as médias das 3 direções de polarização $(x, y, z)$. As linhas verticais tracejadas indicam o band gap eletrônico no ponto $\Gamma$ dos compostos. . . . . . . . . . . . . . . . . . 56

Figura 16 - Representação das estruturas cristalinas 2D-ortorrômbica, 2D-triclínica e 3D-cúbica dos compostos $\mathrm{A}_{2} \mathrm{M}^{\mathrm{II}} \mathrm{M}_{3}^{\mathrm{IV}} \mathrm{Q}_{8} \ldots \ldots \ldots \ldots$

Figura 17 - Energia total relativa das estruturas 2D-triclínica, 3D-cúbica e 2Dortorrômbica dos compostos $\mathrm{A}_{2} \mathrm{ZnSn}_{3} \mathrm{Q}_{8}(\mathrm{~A}=\mathrm{Li}, \mathrm{K}, \mathrm{Cs} ; \mathrm{Q}=\mathrm{S}$, Se, $\mathrm{Te}$ ), em função da média dos raios atômicos de $\mathrm{A}$ e Q. Em (a), os raios atômicos usados são valores de raios iônicos de referência, ${ }^{40}$ e em (b) os raios atômicos foram obtidos a partir dos valores de distâncias médias de ligação, usando o modelo de esferas rígidas, $R=d_{m e d} / 2 \ldots$. . . . .

Figura 18 - Estruturas de bandas dos compostos $\mathrm{Cs}_{2} \mathrm{MgGe}_{3} \mathrm{Q}_{8}(\mathrm{Q}=\mathrm{S}, \mathrm{Se}, \mathrm{Te})$, calculadas com PBE. O topo da banda de valência está deslocado para energia zero. . . . . . . . . . . . . . . . . . . . . . . . 777

Figura 19 - Estruturas de bandas dos compostos $\mathrm{Cs}_{2} \mathrm{MgSn}_{3} \mathrm{Q}_{8}(\mathrm{Q}=\mathrm{S}, \mathrm{Se}, \mathrm{Te})$, calculadas com PBE. O topo da banda de valência está deslocado para energia zero. . . . . . . . . . . . . . . . . . 77 
Figura 20 - Estruturas de bandas dos compostos $\mathrm{Cs}_{2} \mathrm{ZnGe}_{3} \mathrm{Q}_{8}(\mathrm{Q}=\mathrm{S}$, Se, Te), calculadas com PBE. O topo da banda de valência está deslocado para energia zero. . . . . . . . . . . . . . . . . . . 78

Figura 21 - Estruturas de bandas dos compostos $\mathrm{Cs}_{2} \mathrm{CdSn}_{3} \mathrm{Q}_{8}(\mathrm{Q}=\mathrm{S}, \mathrm{Se}, \mathrm{Te})$, calculadas com PBE. O topo da banda de valência está deslocado para energia zero. . . . . . . . . . . . . . . . . . 78

Figura 22 - Estruturas de bandas dos compostos $\mathrm{Cs}_{2} \mathrm{HgGe}_{3} \mathrm{Q}_{8}(\mathrm{Q}=\mathrm{S}, \mathrm{Se}, \mathrm{Te})$, calculadas com PBE. O topo da banda de valência está deslocado para energia zero. . . . . . . . . . . . . . . . . . . 79

Figura 23 - Estruturas de bandas dos compostos $\mathrm{Cs}_{2} \mathrm{HgSn}_{3} \mathrm{Q}_{8}(\mathrm{Q}=\mathrm{S}$, Se, Te), calculadas com PBE. O topo da banda de valência está deslocado para energia zero. . . . . . . . . . . . . . . . . . . 79

Figura 24 - Densidade de estados por célula cristalina dos compostos $\mathrm{Cs}_{2} \mathrm{MgGe}_{3} \mathrm{Q}_{8}$ $(\mathrm{Q}=\mathrm{S}, \mathrm{Se}, \mathrm{Te})$, calculadas com PBE. O topo da banda de valência está deslocado para energia zero. . . . . . . . . . . . . . . . 80

Figura 25 - Densidade de estados por célula cristalina dos compostos $\mathrm{Cs}_{2} \mathrm{MgSn}_{3} \mathrm{Q}_{8}$ $(\mathrm{Q}=\mathrm{S}, \mathrm{Se}, \mathrm{Te})$, calculadas com PBE. O topo da banda de valência está deslocado para energia zero. . . . . . . . . . . . . . . . . . 81

Figura 26 - Densidade de estados por célula cristalina dos compostos $\mathrm{Cs}_{2} \mathrm{ZnGe}_{3} \mathrm{Q}_{8}$ $(\mathrm{Q}=\mathrm{S}, \mathrm{Se}, \mathrm{Te})$, calculadas com PBE. O topo da banda de valência está deslocado para energia zero. . . . . . . . . . . . . . 82

Figura 27 - Densidade de estados por célula cristalina dos compostos $\mathrm{Cs}_{2} \mathrm{CdSn}_{3} \mathrm{Q}_{8}$ $(\mathrm{Q}=\mathrm{S}, \mathrm{Se}, \mathrm{Te})$, calculadas com PBE. O topo da banda de valência está deslocado para energia zero. . . . . . . . . . . . . . . . . . 83

Figura 28 - Densidade de estados por célula cristalina dos compostos $\mathrm{Cs}_{2} \mathrm{HgGe}_{3} \mathrm{Q}_{8}$ $(\mathrm{Q}=\mathrm{S}$, Se, Te), calculadas com PBE. O topo da banda de valência está deslocado para energia zero. . . . . . . . . . . . . . . . . . 84

Figura 29 - Densidade de estados por célula cristalina dos compostos $\mathrm{Cs}_{2} \mathrm{HgSn}_{3} \mathrm{Q}_{8}$ $(\mathrm{Q}=\mathrm{S}, \mathrm{Se}, \mathrm{Te})$, calculadas com PBE. O topo da banda de valência está deslocado para energia zero. . . . . . . . . . . . . . . . . 8 85 



\section{LISTA DE TABELAS}

Tabela 1 - Parâmetros de rede de equilíbrio e energias relativas dos compostos $\mathrm{Cs}_{2} \mathrm{CdSn}_{3} \mathrm{Se}_{8}$ e $\mathrm{Cs}_{2} \mathrm{ZnGe}_{3} \mathrm{~S}_{8}$ calculados nas estruturas ortorrômbica e monoclínica. . . . . . . . . . . . . . . . . 40

Tabela 2 - Parâmetros de rede da estrutura ortorrômbica dos materiais $\mathrm{Cs}_{2} \mathrm{M}^{\mathrm{II}} \mathrm{M}_{3}^{\mathrm{IV}} \mathrm{Q}_{8}$, $\left(\mathrm{M}^{\mathrm{II}}=\mathrm{Mg}, \mathrm{Zn}, \mathrm{Cd}, \mathrm{Hg} ; \mathrm{M}^{\mathrm{IV}}=\mathrm{Ge}, \mathrm{Sn} ; \mathrm{Q}=\mathrm{S}\right.$, Se, Te) obtidos com PBE (1) e PBE+TS+SCS (2), e valores experimentais para os materiais com dados disponíveis na literatura. . . . . . . . . . . . . . . . 42

Tabela 3 - Raios atômicos baseados no modelo de esferas-rígidas e valores de $d_{m e d}$ dos elementos químicos presentes nos materiais $\mathrm{Cs}_{2} \mathrm{M}^{\mathrm{II}} \mathrm{M}_{3}^{\mathrm{IV}} \mathrm{Q}_{8}$, obtidos das estruturas ortorrômbicas otimizadas com PBE e PBE+TS+SCS. . 44

Tabela 4 - Valores de eletronegatividade de Pauling dos elementos químicos presentes nos materiais $\mathrm{Cs}_{2} \mathrm{M}^{\mathrm{II}} \mathrm{M}_{3}^{\mathrm{IV}} \mathrm{Q}_{8}$. . . . . . . . . . . . . . . . 47

Tabela 5 - Parâmetros de rede de equilíbrio e energias totais relativas dos materiais $\mathrm{A}_{2} \mathrm{ZnSn}_{3} \mathrm{Q}_{8}(\mathrm{~A}=\mathrm{Li}, \mathrm{K}, \mathrm{Cs} ; \mathrm{Q}=\mathrm{S}, \mathrm{Se}, \mathrm{Te})$ nas estruturas 2D-triclínica, 3D-cúbica e 2D-ortorrômbica . . . . . . . . . . . . . . . 61

Tabela 6 - Comparação entre os resultados dos parâmetros de rede obtidos nesse trabalho e os dados experimentais de $\mathrm{K}_{2} \mathrm{ZnSn}_{3} \mathrm{~S}_{8}$ nas estruturas 2Dtriclínica e 3D-cúbica, e estrutura 2D-ortorrômbica de $\mathrm{Cs}_{2} \mathrm{ZnSn}_{3} \mathrm{~S}_{8}$. . .

Tabela 7 - Resultados de números de coordenação efetiva, NCE, e distâncias médias de ligação, $\mathrm{d}_{\text {med }}$, dos 24 compostos $\mathrm{Cs}_{2} \mathrm{M}^{\mathrm{II}} \mathrm{M}_{3}^{\mathrm{IV}} \mathrm{Q}_{8}\left(\mathrm{M}^{\mathrm{II}}=\mathrm{Mg}, \mathrm{Zn}, \mathrm{Cd}\right.$, $\mathrm{Hg} ; \mathrm{M}^{\mathrm{IV}}=\mathrm{Ge}, \mathrm{Sn} ; \mathrm{Q}=\mathrm{S}, \mathrm{Se}, \mathrm{Te}$ ) na estrutura ortorrômbica otimizada com PBE. . . . . . . . . . . . . . . . . . . . . 75

Tabela 8 - Resultados de números de coordenação efetiva, NCE, e distâncias médias de ligação, $\mathrm{d}_{m e d}$, dos 24 compostos $\mathrm{Cs}_{2} \mathrm{M}^{\mathrm{II}} \mathrm{M}_{3}^{\mathrm{IV}} \mathrm{Q}_{8}\left(\mathrm{M}^{\mathrm{II}}=\mathrm{Mg}, \mathrm{Zn}, \mathrm{Cd}\right.$, $\mathrm{Hg} ; \mathrm{M}^{\mathrm{IV}}=\mathrm{Ge}, \mathrm{Sn} ; \mathrm{Q}=\mathrm{S}, \mathrm{Se}, \mathrm{Te}$ ) na estrutura ortorrômbica otimizada com PBE+TS+SCS. . . . . . . . . . . . . . . . 76 

INTRODUÇÃO $\ldots \ldots \ldots \ldots \ldots \ldots \ldots$

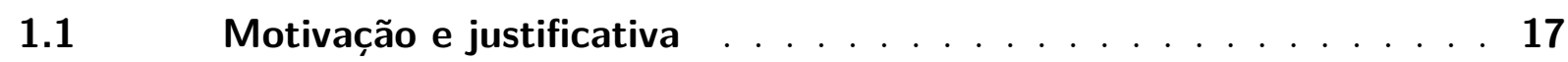

$1.2 \quad$ Calcogenetos quaternários $\mathrm{A}_{2} \mathrm{M}^{\mathrm{II}} \mathrm{M}_{3}^{\mathrm{IV}} \mathrm{Q}_{8} \ldots \ldots \ldots \ldots \ldots$

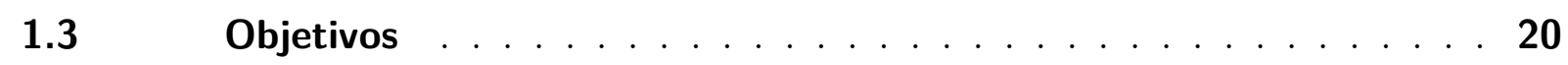

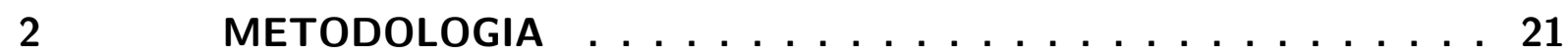

$2.1 \quad$ Problema de muitos corpos e aproximação de Born-Oppenheimer . 21

2.2 Teoria do funcional da densidade . . . . . . . . . . . . 23

2.2.1 Teoremas de Hohenberg-Kohn . . . . . . . . . . . . . . 23

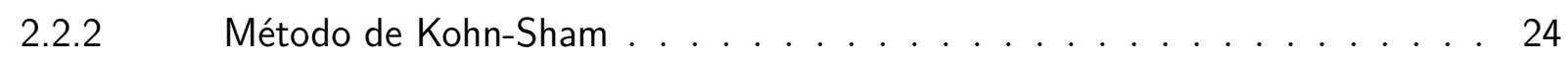

2.2.3 Funcionais de troca e correlação . . . . . . . . . . . . . . 26

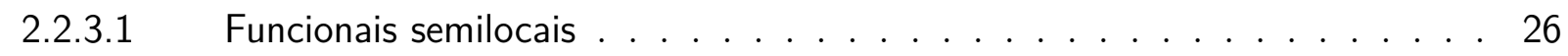

2.2.3.2 Funcionais híbridos . . . . . . . . . . . . . . . . . . 27

$2.2 .4 \quad$ Correções de van der Waals . . . . . . . . . . . . . . . . 28

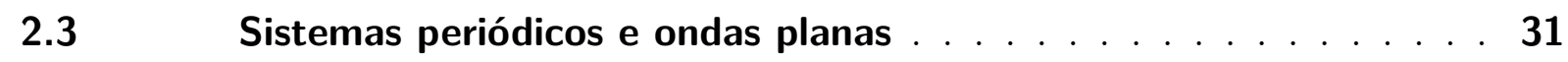

$2.4 \quad$ Método Projector Augmented-Wave . . . . . . . . . . . 33

2.5 Detalhes computacionais e testes de convergência . . . . . . 35

3 PROPRIEDADES ESTRUTURAIS, ELETRÔNICAS E ÓPTICAS DOS CALCOGENETOS QUATERNÁRIOS $\mathrm{Cs}_{2} \mathrm{M}^{\prime \prime} \mathrm{M}_{3}^{\mathrm{IV}} \mathbf{Q}_{8} \ldots \ldots . .39$

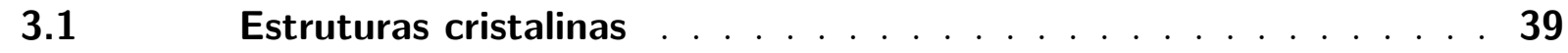

3.1.1 Comparação entre as estruturas ortorrômbica e monoclínica . . . . . . . 39

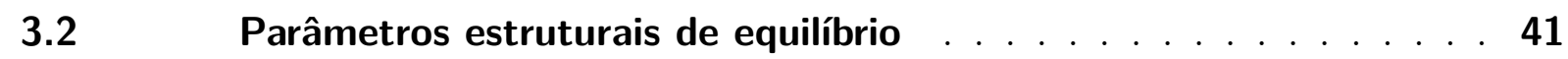

3.2.1 Coordenação efetiva . . . . . . . . . . . . . . 43

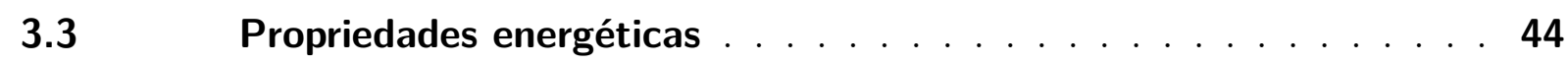

3.3.1 Energia de coesão . . . . . . . . . . . . . . . . . 45

3.3.1.1 Análise das cargas efetivas . . . . . . . . . . . . . . 46

3.3.2 Energia de ligação entre camadas . . . . . . . . . . . . . . 47

$3.4 \quad$ Propriedades eletrônicas $\ldots \ldots \ldots \ldots$

3.4.1 Estrutura de bandas . . . . . . . . . . . . . . . . 49

3.4.2 Densidade de estados . . . . . . . . . . . . . . . 51

3.4.3 Band gaps . . . . . . . . . . . . . . . . 53

$3.5 \quad$ Propriedades ópticas $\ldots \ldots \ldots \ldots \ldots \ldots \ldots \ldots \ldots \ldots \ldots$

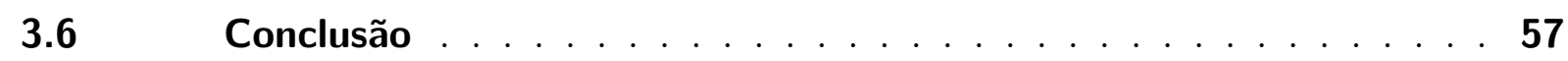


EFEITO DOS ÁTOMOS DE METAIS ALCALINOS E CALCOGÊNIOS NA ESTABILIDADE RELATIVA DAS ESTRUTURAS CRISTALINAS DOS CALCOGENETOS QUATERNÁRIOS $\mathrm{A}_{2} \mathbf{Z n S n}_{3} \mathbf{Q}_{8}$. 59

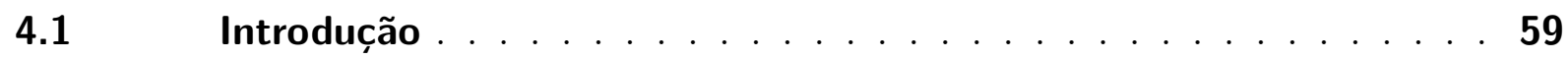

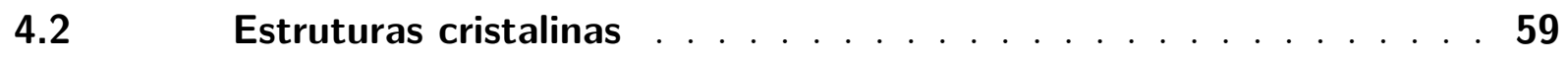

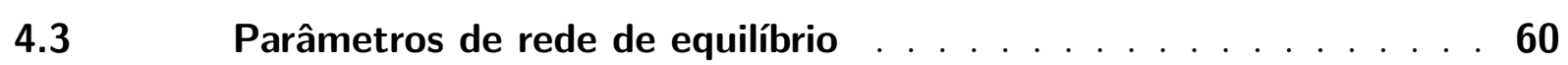

$4.4 \quad$ Estabilidade relativa das estruturas . . . . . . . . . . 61

$4.5 \quad$ Conclusão . . . . . . . . . . . . . . . . . . . 64

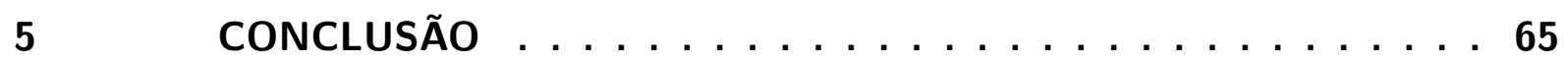

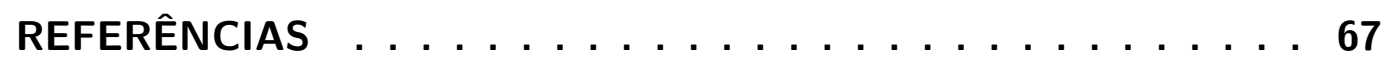

$\begin{array}{ll}\text { APÊNDICES } & 73\end{array}$

APÊNDICE A - RESULTADOS SUPLEMENTARES DAS PROPRIEDADES ESTRUTURAIS, ELETRÔNICAS E ÓPTICAS DOS CALCOGENETOS QUATERNÁRIOS $\mathrm{CS}_{2} \mathrm{M}^{\mathrm{II}} \mathrm{M}_{3}^{\mathrm{IV}} \mathbf{Q}_{8} \ldots \ldots \ldots \ldots \ldots \ldots$ 


\section{INTRODUÇÃO}

\subsection{Motivação e justificativa}

Calcogenetos, os compostos com ao menos um ânion calcogênio ( $\mathrm{S}, \mathrm{Se}, \mathrm{Te}$ ), existem em uma grande variedade de composições químicas, como calcogenetos binários $\mathrm{M}_{m}^{p} \mathrm{Q}_{n}^{-2}$ (onde $p \times m-2 \times n=0$ ) ou calcogenetos ternários e quaternários, obtidos com diferentes espécies químicas substituindo o cátion M. Devido a esse número de composições e também às diferentes estruturas cristalinas que elas possuem, uma vasta diversidade de propriedades físicas e químicas pode ser obtida com esses materiais. Por isso, vários calcogenetos se mostraram úteis para muitas aplicações tecnológicas, como células solares, ${ }^{1-3}$ armazenamento de dados, ${ }^{4-5}$ remoção de resíduos nucleares ${ }^{6}$ e materiais ópticos nãolineares ${ }^{7-8}$ e, além disso, outras propriedades têm sido exploradas através da síntese e caracterização de novos materiais. ${ }^{9-10}$

Dentre os compostos mais simples, os calcogenetos binários, podem ser destacados CdTe e CdS, os quais têm características propícias para utilização em dispositivos fotovoltaicos, entre as quais estão o band gap do CdTe, próximo do ideal para esse fim $(1,50 \mathrm{eV})$, o elevado coeficiente de absorção óptica de CdTe, o alinhamento das bandas de CdS e CdTe e existências de técnicas que permitem a produção em larga escala com custo adequado. ${ }^{1,11}$ Além desses materiais, os dicalcogenetos de metais de transição, em especial $\mathrm{MoQ}_{2}(\mathrm{Q}=\mathrm{S}$, Se), também têm atraído grande interesse, devido à crescente importância da pesquisa em materiais bidimensionais (2D). ${ }^{12-13}$ Os materiais bidimensionais, definidos por camadas de átomos que são empilhadas na formação das estruturas tridimensionais, a exemplo das camadas de grafeno que constituem o grafite, têm sido muito estudados por exibirem novas propriedades e fenômenos em relação aos sólidos usuais, indicando potencial para novas aplicações, ${ }^{13-14}$ bem como pelo interesse na construção de heteroestruturas de van der Waals, que são empilhamentos de camadas de compostos diversos e possibilitam projetar materiais com características específicas. ${ }^{14-15}$ Nesse contexto, buscam-se diferentes compostos, a fim de explorar novas propriedades, como materiais $2 \mathrm{D}$ que, diferentemente do grafeno, tenham band gap.

Além dos vários calcogenetos binários, a diversidade de propriedades entre os calcogenetos é igualmente observada nas composições mais complexas, como os compostos ternários e quaternários. Neles, as características da estrutura cristalina também têm papel importante na determinação das propriedades do material, como nos sulfetos quaternários $\mathrm{K}_{2 x} \mathrm{Mn}_{x} \mathrm{Sn}_{3-x} \mathrm{~S}_{6}(x=0,5-0,95)$. Na estrutura cristalina desses materiais, os íons $\mathrm{K}^{+}$ são localizados em posições desordenadas entre camadas formadas por $\mathrm{Mn}, \mathrm{Sn}, \mathrm{S},{ }^{6}$ o que favorece a mobilidade desses íons, facilitando reações de troca iônica e, portanto, esses materiais são bons candidatos para aplicação em remoção de resíduos nucleares. ${ }^{6}$ Outros 
calcogenetos ternários e quaternários também possuem estruturas cristalinas formadas por camadas, como $\mathrm{Tl}_{2} \mathrm{Hg}_{3} \mathrm{Q}_{4}(\mathrm{Q}=\mathrm{S}, \mathrm{Se}, \mathrm{Te}),{ }^{9}$ e $\mathrm{NaGaGe}_{3} \mathrm{Se}_{8} \cdot{ }^{10}$ Entre os calcogenetos quaternários, similarmente se destaca o semicondutor CuInGaSe ${ }_{2}$ (CIGS), que é utilizado em células solares de alto rendimento, ${ }^{2}$ e os materiais $\mathrm{Cu}_{2} \mathrm{ZnSnQ}_{4}(\mathrm{Q}=\mathrm{S}, \mathrm{Se})$, com estrutura cristalina similar a CIGS, que também possuem propriedades adequadas para aplicação em células solares e, por isso, são estudados como alternativas, por exemplo, ao custo e limitação do In na produção de CIGS. ${ }^{3}$ Também existem semicondutores calcogenetos com as características adequadas para aplicação em detectores de radiação de altas energias, isto é, com alta densidade de massa e band gap largo (> 1,5 eV), como $\mathrm{CsCdInQ}_{3}(\mathrm{Q}=\mathrm{Se}, \mathrm{Te}) .{ }^{16}$ Portanto, os calcogenetos quaternários oferecem a possibilidade de obter várias propriedades específicas de interesse, além de poderem gerar materiais bidimensionais e, consequentemente, existe um grande esforço para obter e caracterizar novos materiais calcogenetos. Então, é importante desenvolver um entendimento, inclusive a nível atomístico, de como a composição química se correlaciona com a estrutura cristalina e outras características do material.

\subsection{Calcogenetos quaternários $A_{2} M^{\prime \prime} M_{3}^{I V} Q_{8}$}

A formação dos calcogenetos ternários e quaternários gera modificações e maior diversidade nas estruturas cristalinas em relação aos compostos binários. Por exemplo, com a introdução de metais alcalinos (K, Cs) na estrutura de calcogenetos binários, como CdTe e HgSe, ocorre redução na dimensionalidade da estrutura cristalina desses materiais. As estruturas de HgSe e CdTe são redes tridimensionais fechadas (estruturas tipo 3D), mas, para acomodar na estrutura os íons originários de $\mathrm{AQ}_{2}(\mathrm{Q}=$ calcogênio $)$ as estruturas são modificadas e adquirem uma dimensionalidade menor, isto é, com rede tridimensional menos compacta ou formadas por camadas e cadeias unidimensionais, por exemplo. ${ }^{17}$ Dessa forma, os materiais formados, como $\mathrm{Cs}_{2} \mathrm{Hg}_{6} \mathrm{~S}_{7}$ e $\mathrm{Cs}_{2} \mathrm{Cd}_{3} \mathrm{Te}_{4}$, têm band gaps mais largos o que pode torná-los apropriados para utilização em detecção de radiação de altas energias. Similarmente, os compostos quaternários $\mathrm{CsCdInQ}_{3}(\mathrm{Q}$ $=\mathrm{Se}, \mathrm{Te}$ ) têm diferenças estruturais em relação aos compostos ternários $\mathrm{CsIn}_{2}$, que resultam em band gaps menores. ${ }^{16}$ Estruturas com diferentes dimensionalidades $(2 \mathrm{D}$ e 3D) também são observadas em famílias de compostos muito parecidos. Por exemplo, enquanto $\mathrm{LiGaGe}_{2} \mathrm{Se}_{6}$ tem uma estrutura cristalina tipo $3 \mathrm{D},{ }^{18}$ os compostos $\mathrm{NaGaGe}_{3} \mathrm{Se}_{8}$, $\mathrm{K}_{3} \mathrm{Ga}_{3} \mathrm{Ge}_{7} \mathrm{~S}_{20}$ e $\mathrm{K}_{3} \mathrm{Ga}_{3} \mathrm{Ge}_{7} \mathrm{Se}_{20}$, têm estruturas em camadas, devido ao maior tamanho e ionicidade dos átomos de metais alcalinos. ${ }^{10}$ Isso demonstra como a síntese de calcogenetos complexos permite explorar vários tipos de estruturas cristalinas.

Como parte da busca por novos materiais calcogenetos, recentemente foram reportados 12 calcogenetos de composição $\mathrm{A}_{2} \mathrm{M}^{\mathrm{II}} \mathrm{M}_{3}^{\mathrm{IV}} \mathrm{Q}_{8}$, com $\mathrm{A}=\mathrm{K}$, Cs; $\mathrm{M}^{\mathrm{II}}=\mathrm{Mg}, \mathrm{Zn}$, $\mathrm{Cd}, \mathrm{Hg} ; \mathrm{M}^{\mathrm{IV}}=\mathrm{Ge}$, Sn e Q $=\mathrm{S}$, Se, Te. ${ }^{19-20}$ Nessa família de materiais observam-se dois 
tipos de estruturas, sendo uma formada pelo empilhamento de camadas (2D), e outra sem essa característica (3D). Além disso, dentre os 11 compostos com $\mathrm{A}=\mathrm{Cs}$ uma grande diversidade de band gaps foi observada, isto é, com valores que variam entre 1,07 eV e 3,38 eV . Dessa forma, estudando essa família de calcogenetos é possível analisar o efeito da composição química na estabilidade relativa das estruturas cristalinas, assim como avaliar como as propriedades, em especial o band gap, podem ser ajustadas com a variação da composição.

O composto $\mathrm{K}_{2} \mathrm{ZnSn}_{3} \mathrm{~S}_{8}$ foi a primeiro a ser sintetizado da família $\mathrm{A}_{2} \mathrm{M}^{\mathrm{II}} \mathrm{M}_{3}^{\mathrm{IV}} \mathrm{Q}_{8}$, em um estudo motivado pela busca de um análogo contendo $\mathrm{Zn}$ para $\mathrm{K}_{2 x} \mathrm{Mn}_{x} \mathrm{Sn}_{3-x} \mathrm{~S}_{6} \cdot{ }^{19}$ A característica de destaque desse composto é que, em seu processo de síntese, duas fases distintas são obtidas. Com resfriamento rápido, obtém-se uma estrutura amorfa, enquanto o resfriamento lento produz uma estrutura cristalina com rede triclínica, que é formada pelo empilhamento de camadas de $\mathrm{ZnSn}_{3} \mathrm{~S}_{8}$, entre as quais estão posicionados os átomos de K. As duas formas são convertidas para uma outra estrutura cristalina quando passam por tratamento térmico, o que permite concluir que essa última é a fase termodinamicamente estável. Essa fase tem estrutura cristalina com rede cúbica e é formada por tetraedros de $\mathrm{MQ}_{4}(\mathrm{M}=\mathrm{Zn}, \mathrm{Sn})$ que se ligam por compartilhamento de vértices, compondo uma rede tridimensional, sem empilhamento de camadas, enquanto os átomos de $\mathrm{K}$ ocupam sítios entre os tetraedros $\mathrm{MQ}_{4}$.

Foram sintetizados 11 compostos $\mathrm{Cs}_{2} \mathrm{M}^{\mathrm{II}} \mathrm{M}_{3}^{\mathrm{IV}} \mathrm{Q}_{8}: \mathrm{Cs}_{2} \mathrm{MgGe}_{3} \mathrm{Se}_{8}, \mathrm{Cs}_{2} \mathrm{MgSn}_{3} \mathrm{Se}_{8}$, $\mathrm{Cs}_{2} \mathrm{ZnGe}_{3} \mathrm{~S}_{8}, \mathrm{Cs}_{2} \mathrm{ZnGe}_{3} \mathrm{Se}_{8}, \mathrm{Cs}_{2} \mathrm{ZnGe}_{3} \mathrm{Te}_{8}, \mathrm{Cs}_{2} \mathrm{ZnSn}_{3} \mathrm{Se}_{8}, \mathrm{Cs}_{2} \mathrm{CdGe}_{3} \mathrm{~S}_{8}, \mathrm{Cs}_{2} \mathrm{CdGe}_{3} \mathrm{Se}_{8}$, $\mathrm{Cs}_{2} \mathrm{CdSn}_{3} \mathrm{Se}_{8}, \mathrm{Cs}_{2} \mathrm{HgSn}_{3} \mathrm{~S}_{8}$ e $\mathrm{Cs}_{2} \mathrm{HgSn}_{3} \mathrm{~S}_{8} .{ }^{20}$ Todos os compostos cristalizam em uma estrutura com rede ortorrômbica, com exceção de $\mathrm{Cs}_{2} \mathrm{ZnGe}_{3} \mathrm{~S}_{8}$, que tem estrutura cristalina com rede monoclínica. Essas duas estruturas são muito similares à estrutura triclínica de $\mathrm{K}_{2} \mathrm{ZnSn}_{3} \mathrm{~S}_{8}$, ou seja, também são caracterizadas pela formação de camadas, sendo que a principal distinção entre as três estruturas está no empilhamento das camadas, isto é, em deslocamentos de átomos de uma camada em relação aos seus correspondentes nas camadas mais próximas. Diferentemente do observado em $\mathrm{K}_{2} \mathrm{ZnSn}_{3} \mathrm{~S}_{8}$, não ocorrem transformações estruturais nos compostos $\mathrm{Cs}_{2} \mathrm{M}^{\mathrm{II}} \mathrm{M}_{3}^{\mathrm{IV}} \mathrm{Q}_{8}$ quando eles passam por tratamento térmico. As medidas de band gap mostraram que os valores variam de $1,07 \mathrm{eV}$ a $3,38 \mathrm{eV}$ entre os semicondutores $\mathrm{Cs}_{2} \mathrm{M}^{\mathrm{II}} \mathrm{M}_{3}^{\mathrm{IV}} \mathrm{Q}_{8}$, ou seja, a composição química pode ser usada para manipular o band gap nessa família de compostos, o que sugere diversas aplicações eletrônicas. Alguns poucos cálculos de estrutura eletrônica já foram reportados para essa família de calcogenetos, apenas para os compostos $\mathrm{Cs}_{2} \mathrm{MgGe}_{3} \mathrm{Se}_{8}, \mathrm{Cs}_{2} \mathrm{MgSn}_{3} \mathrm{Se}_{8}, \mathrm{Cs}_{2} \mathrm{ZnGe}_{3} \mathrm{Se}_{8}$ e $\mathrm{Cs}_{2} \mathrm{ZnSn}_{3} \mathrm{Se}_{8} \cdot{ }^{20}$ Além disso, como esses cálculos foram feitos com base na aproximação da densidade local na teoria do funcional da densidade, eles subestimaram consideravelmente os band gaps, de forma que os autores sugeriram métodos mais sofisticados para estudos futuros. ${ }^{20}$ 
Considerando todos os elementos químicos utilizados para cada componente de $\mathrm{Cs}_{2} \mathrm{M}^{\mathrm{II}} \mathrm{M}_{3}^{\mathrm{IV}} \mathrm{Q}_{8}$, isto é, $\mathrm{M}^{\mathrm{II}}=\mathrm{Mg}, \mathrm{Zn}, \mathrm{Cd}, \mathrm{Hg} ; \mathrm{M}^{\mathrm{IV}}=\mathrm{Ge}$, Sn e $\mathrm{Q}=\mathrm{S}$, Se, Te, existe um total de 24 possíveis compostos, dos quais apenas 11 foram estudados experimentalmente. Portanto, a fim de compreender melhor as propriedades dessa família de calcogenetos, e quais são os efeitos da modificação da composição, é importante estender a caracterização para todos os 24 compostos possíveis. Também chama a atenção o fato de uma estrutura $3 \mathrm{D}$ ser observada para o composto $\mathrm{K}_{2} \mathrm{ZnSn}_{3} \mathrm{~S}_{8}$ e não para os compostos com Cs, o que indica que os átomos de metais alcalinos são importantes na determinação da estabilidade relativa dessas estruturas e, portanto, é necessário investigar mais os efeitos da composição química na estabilidade das estruturas desses materiais.

\subsection{Objetivos}

Tendo em vista a importância dos calcogenetos no desenvolvimento de novos materiais para avanços científicos, como no caso de materiais bidimensionais, e também para diversas aplicações tecnológicas, conforme descrito nas seções anteriores, é necessário obter um melhor entendimento atomístico dos fatores que afetam as propriedades desses materiais. Como a família de calcogenetos $\mathrm{A}_{2} \mathrm{M}^{\mathrm{II}} \mathrm{M}_{3}^{\mathrm{IV}} \mathrm{Q}_{8}$ possui diversidade de tipos de estrutura cristalina e de propriedades dependendo da composição química, ela pode ser tomada como exemplo importante para examinar os efeitos da composição na determinação dessas propriedades. Assim, para estudar essas questões, esse trabalho utiliza uma abordagem teórica através de cálculos com a teoria do funcional da densidade, em que os principais objetivos são definidos da seguinte forma:

1. Determinar propriedades estruturais, energéticas, eletrônicas e ópticas dos 24 compostos $\mathrm{Cs}_{2} \mathrm{M}^{\mathrm{II}} \mathrm{M}_{3}^{\mathrm{IV}} \mathrm{Q}_{8}$, e avaliar o efeito da composição química nessas propriedades.

2. Estudar o efeito dos átomos de metais alcalinos e calcogênios na estabilidade relativa das estruturas de $\mathrm{A}_{2} \mathrm{M}^{\mathrm{II}} \mathrm{M}_{3}^{\mathrm{IV}} \mathrm{Q}_{8}$.

No capítulo 2, são descritos os fundamentos da metodologia empregada, enquanto nos capítulos 3 e 4 são apresentados os resultados referentes aos itens 1 e 2 acima, respectivamente. Por fim, o capítulo 5 contém um sumário das principais conclusões obtidas nesse trabalho. 


\section{METODOLOGIA}

Esse capítulo é dividido em quatro partes: (2.1) Problema de muitos corpos e aproximação de Born-Oppenheimer; (2.2) Teoria do funcional da densidade; (2.3) Sistemas periódicos e ondas planas; (2.4) Método Projector Augmented-Wave.

\subsection{Problema de muitos corpos e aproximação de Born-Oppenheimer}

Nesse trabalho busca-se desenvolver um entendimento atomístico das propriedades dos compostos $\mathrm{A}_{2} \mathrm{M}^{\mathrm{II}} \mathrm{M}_{3}^{\mathrm{IV}} \mathrm{Q}_{8}$. Além disso, a caracterização eletrônica e óptica de sólidos envolve o conhecimento dos estados eletrônicos. Portanto, nesse trabalho os materiais são estudados com base em uma descrição quântica dos sistemas de partículas que os formam. Então, considera-se o sistema de muitos corpos ( $N$ elétrons e $M$ núcleos), cujo hamiltoniano não-relativístico é dado por,

$$
\hat{H}=\hat{V}_{N e}+\hat{V}_{e e}+\hat{T}_{e}+\hat{V}_{N N}+\hat{T}_{N}
$$

O primeiro termo é a contribuição da interação coulombiana entre núcleos e elétrons,

$$
\hat{V}_{N e}=\sum_{I=1}^{M} \sum_{i=1}^{N} \frac{-Z_{I}}{\left|\mathbf{R}_{I}-\mathbf{r}_{i}\right|},
$$

onde $\mathbf{R}_{I}$ e $\mathbf{r}_{i}$ são as posições de núcleos e elétrons, respectivamente, e $Z_{I}$ são os números atômicos dos núcleos. Aqui, utiliza-se o sistema de unidades atômicas $\left(m_{e}=e=\hbar=\right.$ $\left.1 / 4 \pi \epsilon_{0}=1\right)$. O termo $\hat{V}_{e e}$ corresponde à interação coulombiana entre elétrons,

$$
\hat{V}_{e e}=\sum_{i=1}^{N} \sum_{j<i} \frac{1}{\left|\mathbf{r}_{i}-\mathbf{r}_{j}\right|} .
$$

O termo $\hat{T}_{e}$ descreve a energia cinética dos elétrons,

$$
\hat{T}_{e}=-\frac{1}{2} \sum_{i=1}^{N} \nabla_{\mathbf{r}_{i}}^{2}
$$

o quarto termo refere-se à repulsão coulombiana entre os núcleos,

$$
\hat{V}_{N N}=\sum_{I=1}^{M} \sum_{J<I} \frac{Z_{I} Z_{J}}{\left|\mathbf{R}_{I}-\mathbf{R}_{J}\right|} .
$$

e, por fim, a energia cinética dos núcleos é incluída pelo quinto termo,

$$
\hat{T}_{N}=-\frac{1}{2} \sum_{I=1}^{M} \frac{1}{M_{I}} \nabla_{\mathbf{R}_{I}}^{2},
$$


onde $M_{I}$ é a massa do $i$-ésimo núcleo. Em princípio, as propriedades do sistema podem ser obtidas a partir da função de onda do sistema, $\Psi(\{\mathbf{r}\},\{\mathbf{R}\})$, onde $\{\mathbf{r}\}$ e $\{\mathbf{R}\}$ denotam os conjuntos de $\mathbf{r}_{i}$ e $\mathbf{R}_{I}$. Essa função é a solução da equação de Schrödinger,

$$
\hat{H} \Psi(\{\mathbf{r}\},\{\mathbf{R}\})=E \Psi(\{\mathbf{r}\},\{\mathbf{R}\})
$$

No entanto, soluções analíticas da equação de Schrödinger somente podem ser obtidas em casos simples ou alguns sistemas modelo (átomo de hidrogênio, oscilador harmônico, poço de potencial, etc), de forma que para sistemas complexos o problema é resolvido através de cálculos numéricos. Além disso, algumas aproximações são geralmente utilizadas nos cálculos a fim de viabilizar a obtenção de soluções.

Uma aproximação amplamente utilizada em cálculos de problemas de muitos corpos é a aproximação de Born-Oppenheimer, ${ }^{21}$ que consiste em separar o movimento eletrônico do movimento dos núcleos. Essa aproximação é motivada pela grande diferença entre as massas de núcleos e elétrons, já que a massa do próton é aproximadamente 1836 vezes maior que a massa do elétron. Devido a essa diferença, considera-se que a configuração eletrônica se ajusta quase instantaneamente a cada arranjo de posições dos núcleos, que por serem muito mais massivos movem-se em uma escala de tempo maior, ou seja, o movimento eletrônico é desacoplado do movimento dos núcleos. Essa aproximação é realizada pela proposta de uma função de onda separada entre parte eletrônica e parte nuclar, ou seja,

$$
\Psi(\{\mathbf{r}\},\{\mathbf{R}\})=\chi(\{\mathbf{R}\}) \psi_{\text {ele }}\left(\{\mathbf{r}\},\{\mathbf{R}\}^{\prime}\right)
$$

onde $\chi(\{\mathbf{R}\})$ é a parte nuclear e $\psi_{\text {ele }}\left(\{\mathbf{r}\},\{\mathbf{R}\}^{\prime}\right)$ é a parte eletrônica, sendo que $\{\mathbf{R}\}^{\prime}$ denota a dependência apenas paramétrica em relação às posições dos núcleos. Essa forma aplicada na equação de Schrödinger leva à uma equação para a parte nuclear e uma equação para a parte eletrônica, que é dada por

$$
\left(\hat{V}_{N e}+\hat{V}_{e e}+\hat{T}_{e}\right) \psi_{\text {ele }}\left(\{\mathbf{r}\},\{\mathbf{R}\}^{\prime}\right)=E_{\text {ele }} \psi_{\text {ele }}\left(\{\mathbf{r}\},\{\mathbf{R}\}^{\prime}\right)
$$

A solução dessa equação fornece uma configuração eletrônica de estado fundamental para um conjunto de posições nucleares $\{\mathbf{R}\}$. Com a aproximação de núcleos fixos, a energia total do sistema é obtida somando à $E_{\text {ele }}$ o termo constante de repulsão coulombiana entre os núcleos.

O uso da aproximação de Born-Oppenheimer leva a bons resultados para uma grande quantidade de sistemas. Porém, a aproximação não é adequada em sistemas nos quais há forte acoplamento entre estados eletrônicos e movimentos vibracionais, o que inviabiliza a separação proposta, ou ainda quando se pretende descrever fenômenos nos quais a velocidade dos núcleos não pode ser desprezada. 


\subsection{Teoria do funcional da densidade}

É possível destacar duas estratégias distintas que são utilizadas para determinar a configuração eletrônica a partir do hamiltoniano da equação 2.9. Uma estratégia busca descrever essa configuração através da determinação de uma função de onda dos $N$ elétrons. Métodos baseados em funções de onda pós-Hartree-Fock, como Configuration Interaction e Coupled Cluster, que podem fornecer bons resultados, possuem um elevado custo computacional quando são aplicados a sistemas com um grande número de átomos, o que pode tornar sua aplicação nesses casos inviável.

Uma outra abordagem para tratar o problema é usar a densidade eletrônica como variável fundamental. Essa estratégia tem suas origens no modelo de Thomas-Fermi, ${ }^{22}$ que propôs uma expressão para a energia de um sistema atômico em função da densidade eletrônica. A aplicação do método de Thomas-Fermi possui algumas falhas, devido às aproximações usadas em sua construção, entre as quais destaca-se a sua inaptidão em descrever ligações moleculares, pois sempre fornece energias maiores para moléculas ligadas do que para os átomos que a constituem separadamente. ${ }^{23}$ A fundamentação teórica que possibilitou o avanço da estratégia que se baseia na densidade eletrônica foi fornecida pelos teoremas de Hohenberg e Kohn, ${ }^{24}$ que constituem a base da Teoria do Funcional da Densidade (em inglês, Density Functional Theory, DFT). Posteriormente, o método desenvolvido por Kohn e Sham ${ }^{25}$ viabilizou a realização de cálculos baseados em DFT, que são aplicados nesse trabalho.

\subsubsection{Teoremas de Hohenberg-Kohn}

Teorema 1 O potencial externo de um sistema de muitos elétrons é um funcional único da densidade eletrônica do estado fundamental.

Esse teorema mostra que dada uma densidade eletrônica do estado fundamental em um sistema de muitos elétrons, existe um único potencial externo que pode dar origem à essa densidade. Dessa forma, o hamiltoniano do sistema é fixado pela densidade eletrônica e consequentemente todas as propriedades do sistema também são. Portanto, a densidade eletrônica $n(\mathbf{r})$ pode ser usada como variável fundamental na descrição do sistema.

Teorema 2 A energia $E[\tilde{n}(\mathbf{r})]$ assume o valor minimo apenas quando $\tilde{n}(\mathbf{r})$ for a densidade correta do estado fundamental, ou seja,

$$
E_{0} \leq E[\tilde{n}(\mathbf{r})]
$$

onde $E_{0}$ é a energia do estado fundamental. 
Esse resultado é um análogo do princípio variacional aplicado à densidade eletrônica, mostrando que a densidade eletrônica do estado fundamental minimiza a energia do sistema como funcional da densidade $(E[\tilde{n}(\mathbf{r})])$.

Pelo primeiro teorema, a energia pode ser escrita como funcional da densidade,

$$
E[n(\mathbf{r})]=T_{e}[n(\mathbf{r})]+V_{e e}[n(\mathbf{r})]+V_{N e}[n(\mathbf{r})]=F_{H K}[n(\mathbf{r})]+V_{N e}[n(\mathbf{r})]
$$

onde $F_{H K}$ é o chamado funcional universal de Hohenberg-Kohn, que inclui os termos de energia cinética e interações entre os elétrons, não dependendo do sistema considerado. Para o potencial externo correspondente à interações com $M$ núcleos atômicos, a energia pode ser escrita da seguinte forma,

$$
E[n(\mathbf{r})]=F_{H K}[n(\mathbf{r})]+\int\left(\sum_{I=1}^{M} \frac{-Z_{I}}{\left|\mathbf{R}_{I}-\mathbf{r}\right|}\right) n(\mathbf{r}) d \mathbf{r}=F_{H K}[n(\mathbf{r})]+\int v(\mathbf{r}) n(\mathbf{r}) d \mathbf{r} .
$$

Então, a energia é minimizada usando o vínculo do número de elétrons,

$$
\delta\left[E[n(\mathbf{r})]-\mu\left(\int n(\mathbf{r}) d \mathbf{r}-N\right)\right]=0
$$

o que leva à equação:

$$
\mu=\frac{\delta F_{H K}[n(\mathbf{r})]}{\delta n(\mathbf{r})}+v(\mathbf{r})
$$

Essa equação fornece um procedimento para obter, em princípio exatamente, a densidade eletrônica do estado fundamental do sistema. No entanto, uma forma satisfatória da energia cinética em $F_{H K}[n(\mathbf{r})]$ não é conhecida, o que inviabiliza a aplicação prática desse procedimento. Um grande avanço para superar essa dificuldade foi proporcionado pelo método de Kohn-Sham, descrito a seguir.

\subsubsection{Método de Kohn-Sham}

No método de Kohn-Sham o funcional $F_{H K}$ é particionado da seguinte forma:

$$
F_{H K}[n(\mathbf{r})]=T_{0}[n(\mathbf{r})]+\frac{1}{2} \iint \frac{n(\mathbf{r}) n\left(\mathbf{r}^{\prime}\right)}{\left|\mathbf{r}-\mathbf{r}^{\prime}\right|} d \mathbf{r} d \mathbf{r}^{\prime}+E_{x c}[n(\mathbf{r})],
$$

onde $T_{0}$ é a energia cinética de um sistema de elétrons não-interagentes que possui a mesma densidade eletrônica do sistema real. Assim, são introduzidos orbitais de um elétron, $\Phi_{n}$, chamados orbitais de Kohn-Sham, e portanto $T_{0}$ pode ser escrita como

$$
T_{0}[n(\mathbf{r})]=\sum_{n=1}^{N}\left\langle\Phi_{n}\left|-\frac{1}{2} \nabla^{2}\right| \Phi_{n}\right\rangle
$$

e além disso a densidade eletrônica está relacionada aos orbitais de Kohn-Sham através de

$$
n(\mathbf{r})=\sum_{n=1}^{N}\left|\Phi_{n}(\mathbf{r})\right|^{2}
$$


O segundo termo da partição de Kohn-Sham para $F_{H K}$ descreve as interações elétronelétron por uma interação eletrostática clássica entre distribuições de carga com densidade $n(\mathbf{r})$. O terceiro termo é denominado termo de troca e correlação e contém as contribuições ainda não incluídas, além de correções para os termos anteriores:

(i) Correção da energia cinética do sistema não-interagente para obter a energia do sistema real;

(ii) Termo de troca, que é a energia relacionada à necessidade de anti-simetria na função de onda dos $N$ elétrons, por serem férmions;

(iii) Termo de correlação, que corresponde à alteração de energia do sistema porque o movimentos dos elétrons é correlacionado, isto é, a posição instantânea de cada elétron afeta o movimento dos demais;

(iv) Correção para a auto-interação eletrônica presente na expressão utilizada para a contribuição das interações coulombianas elétron-elétron.

Não é conhecida uma expressão exata para $E_{x c}$ e portanto várias formulações e aproximações foram construídas para esse termo, constituindo os funcionais de troca e correlação, que são discutidos na próxima seção.

Utilizando a partição de Kohn-Sham, a energia,

$$
E[n(\mathbf{r})]=T_{0}[n(\mathbf{r})]+\frac{1}{2} \iint \frac{n(\mathbf{r}) n\left(\mathbf{r}^{\prime}\right)}{\left|\mathbf{r}-\mathbf{r}^{\prime}\right|} d \mathbf{r} d \mathbf{r}^{\prime}+E_{x c}[n(\mathbf{r})]+\int v(\mathbf{r}) n(\mathbf{r}) d \mathbf{r},
$$

é minimizada usando como vínculo a ortonormalidade dos orbitais de Kohn-Sham,

$$
\delta\left[E[n(\mathbf{r})]-\sum_{n=1}^{N} \sum_{m=1}^{N} \varepsilon_{n m} \int \Phi_{n}^{*}(\mathbf{r}) \Phi_{m}(\mathbf{r}) d \mathbf{r}\right]=0 .
$$

Esse procedimento resulta nas equações de Kohn-Sham,

$$
\left[-\frac{1}{2} \nabla^{2}+v_{e f}(\mathbf{r})\right] \Phi_{n}(\mathbf{r})=\varepsilon_{n} \Phi_{n}(\mathbf{r}), n=1, \ldots, N
$$

onde

$$
v_{e f}(\mathbf{r})=\int \frac{n\left(\mathbf{r}^{\prime}\right)}{\left|\mathbf{r}-\mathbf{r}^{\prime}\right|} d \mathbf{r}^{\prime}+v(\mathbf{r})+v_{x c}(\mathbf{r}),
$$

em que $v_{x c}$, chamado de potencial de troca e correlação, é definido por

$$
v_{x c}(\mathbf{r})=\frac{\delta E_{x c}[n(\mathbf{r})]}{\delta n(\mathbf{r})} .
$$

e a expressão para a energia do sistema eletrônico é

$$
E_{\mathrm{DFT}}=\sum_{n=1}^{N} \varepsilon_{n}-\frac{1}{2} \iint \frac{n(\mathbf{r}) n\left(\mathbf{r}^{\prime}\right)}{\left|\mathbf{r}-\mathbf{r}^{\prime}\right|} d \mathbf{r} d \mathbf{r}^{\prime}-\int v_{x c}(\mathbf{r}) n(\mathbf{r}) d \mathbf{r}+E_{x c}[n(\mathbf{r})] .
$$

As equações de Kohn-Sham, usadas para determinar os $\Phi_{n}$, incluem potenciais que são dependentes de $n(\mathbf{r})$ e $n(\mathbf{r})$, por sua vez, é obtida a partir dos $\Phi_{n}$ pela equação 2.17. 
Portanto, as equações de Kohn-Sham devem ser resolvidas de forma auto-consistente. Partindo de uma densidade inicial, obtém-se $v_{e f}(\mathbf{r})$, que é utilizado na equação 2.20 para obter os orbitais de Kohn-Sham, de onde pode ser obtida uma nova densidade para reiniciar o ciclo. Esse processo deve ser repetido até que um critério de convergência previamente estabelecido seja atingido, como uma variação de energia total menor que um valor especificado. A estratégia para resolver as equações de Kohn-Sham envolve a expansão dos orbitais em um conjunto de funções base, e os vários métodos existentes para obter essas soluções apresentam diferenças do tipo de função que é usado na base e também de como essas funções são modificadas ao longo dos cálculos. Nesse trabalho, os cálculos foram realizados com o pacote computacional VASP (Vienna ab initio simulation package) ${ }^{26-27}$ que utiliza o método de projetor de onda aumentada (em inglês, Projector Augmented Wave, PAW) o qual é descrito na seção 2.4.

\subsubsection{Funcionais de troca e correlação}

\subsubsection{Funcionais semilocais}

Conforme mencionado anteriormente, diversas formulações foram desenvolvidas para o termo de troca e correlação da energia no método de Kohn-Sham. A primeira construção de funcional de troca e correlação foi a aproximação da densidade local (em inglês, local density approximation, LDA), que inclui apenas uma dependência da densidade eletrônica em cada ponto do espaço,

$$
E_{x c}^{\mathrm{LDA}}=\int n(\mathbf{r}) \epsilon_{x c}(n(\mathbf{r})) d \mathbf{r}
$$

onde $\epsilon_{x c}(n(\mathbf{r}))$ pode ser decomposto em uma soma dos termos de troca e correlação. A parte de troca corresponde à forma exata do termo da energia troca em um gás de elétrons homogêneo,

$$
\epsilon_{x}^{L D A}(n(\mathbf{r}))=-\frac{3 k_{F}(n(\mathbf{r}))}{4 \pi}
$$

onde $k_{F}(n(\mathbf{r}))=\left(3 \pi^{2} n(\mathbf{r})\right)^{1 / 3}$ é o número de onda de Fermi. A expressão para a parte de correlação é construída através de interpolações com resultados numéricos de Monte Carlo quântico, levando em conta formas analíticas conhecidas para os limites de altas e baixas densidades do gás de elétrons homogêneo. Dessa forma, existem diferentes parametrizações para o termo de correlação LDA. Destacamos a formulação de Perdew e Wang, ${ }^{28}$

$$
\epsilon_{c}^{P W}(n(\mathbf{r}))=-2 \alpha_{1}\left(1+\alpha_{2} r_{s}\right) \ln \left\{1+\left[2 \alpha_{1}\left(B_{1} r_{s}^{1 / 2}+B_{2} r_{s}+B_{3} r_{s}^{3 / 2}+B_{4} r_{s}^{2}\right)\right]^{-1}\right\}
$$

onde $r_{s}(n(\mathbf{r}))=[3 /(4 \pi n(\mathbf{r}))]^{1 / 3}$ é o raio de Wigner-Seitz. Os parâmetros $\alpha_{1}, B_{1}$ e $B_{2}$ são escolhidos para ajustar a expansão exata no limite de alta densidade, e $\alpha_{2}, B_{3}$ e $B_{4}$ são ajustados para minimizar erros para valores numéricos de cálculos correspondentes a baixas densidades. ${ }^{28}$ 
Um outro nível de aproximação dos funcionais é a aproximação do gradiente generalizado (em inglês, Generalized Gradient Approximation, GGA) que utiliza além da densidade eletrônica o seu gradiente em cada ponto do espaço, isto é,

$$
E_{x c}^{\mathrm{GGA}}=\int f_{x c}(n(\mathbf{r}), \nabla n(\mathbf{r})) d \mathbf{r}
$$

onde também pode ser feita a separação $E_{x c}^{\mathrm{GGA}}=E_{x}^{\mathrm{GGA}}+E_{c}^{\mathrm{GGA}}$. Nesse trabalho foi utilizado o funcional PBE, que é um funcional do tipo GGA proposto por Perdew, Burke e Ernzerhof em 1996, ${ }^{29}$ como uma simplificação do funcional PW91, desenvolvido por Perdew e Wang. ${ }^{30}$ O funcional PW91 foi construído buscando satisfazer ao máximo as condições exatas que devem ser obedecidas pela energia de troca e correlação. Já a construção do funcional PBE levou em consideração apenas as condições mais energeticamente significativas. Isso levou a uma formulação mais simples e que apesar disso fornece essencialmente os mesmos resultados que PW91.

A parte de troca do funcional PBE é

$$
E_{x}^{\mathrm{GGA}}=\int n(\mathbf{r}) \epsilon_{x}^{L D A}(n(\mathbf{r})) F_{X}(s) d \mathbf{r},
$$

onde $s=|\nabla n(\mathbf{r})| / 2 n(\mathbf{r}) k_{F}(n(\mathbf{r}))$ é um gradiente adimensional da densidade. A forma de $F_{X}(s)$ é dada por

$$
F_{X}(s)=1+\kappa-\kappa /\left(1+\mu s^{2} / \kappa\right)
$$

onde $\kappa=0.804$ e $\mu=\beta \pi^{2} / 3$ para $\beta=0.066725$.

A parte de correlação do funcional PBE é

$$
E_{c}^{\mathrm{GGA}}=\int n(\mathbf{r})\left[\epsilon_{c}^{P W}\left(r_{s}\right)+H\left(r_{s}, t\right)\right]
$$

onde $t=|\nabla n(\mathbf{r})| / 2 k_{s}(n(\mathbf{r})) n(\mathbf{r})$ e $k_{s}(n(\mathbf{r}))=\sqrt{4 k_{F}(n(\mathbf{r})) / \pi}$, enquanto $H\left(r_{s}, t\right)$ tem a seguinte forma,

$$
H\left(r_{s}, t\right)=\gamma \ln \left[1+\frac{\beta}{\gamma} t^{2}\left(\frac{1+A t^{2}}{1+A t^{2}+A^{2} t^{4}}\right)\right],
$$

onde

$$
A=\frac{\beta}{\gamma}\left[\exp \left\{-\frac{\epsilon_{c}^{P W}}{\gamma}\right\}-1\right]^{-1}, \gamma=(1-\ln 2) / \pi^{2} .
$$

$\beta$ têm o mesmo valor que na parametrização da troca e $\epsilon_{c}^{P W}$ é a parametrização de Perdew e Wang da LDA para correlação.

\subsubsection{Funcionais híbridos}

Uma importante característica dos funcionais LDA e GGA usuais é que os cálculos que utilizam esses funcionais tendem a subestimar consideravelmente o band gap de semicondutores e isolantes. ${ }^{31}$ Devido a importância de obter valores realistas de band gap nesse trabalho, também foram realizados cálculos utilizando o funcional híbrido HSE06. ${ }^{32}$ 
Os funcionais híbridos são caracterizados por incluírem, como parte da energia de troca, o termo de troca do método de Hartee-Fock (HF), que é definido por

$$
E_{x}^{\mathrm{HF}}=-\frac{1}{2} \sum_{i} \sum_{j} \iint \Phi_{i}^{*}(\mathbf{r}) \Phi_{j}(\mathbf{r}) \frac{1}{\left|\mathbf{r}-\mathbf{r}^{\prime}\right|} \Phi_{i}\left(\mathbf{r}^{\prime}\right) \Phi_{j}^{*}\left(\mathbf{r}^{\prime}\right) d \mathbf{r} d \mathbf{r}^{\prime}
$$

onde $i$ e $j$ percorrem os orbitais ocupados. A fração de $E_{x}^{\mathrm{HF}}$ incluída no funcional HSE06 se baseia na formulação do funcional híbrido PBE0, que é definido por

$$
E_{x c}^{\mathrm{PBE} 0}=\frac{1}{4} E_{x}^{\mathrm{HF}}+\frac{3}{4} E_{x}^{\mathrm{PBE}}+E_{c}^{\mathrm{PBE}}
$$

ou seja, com $25 \%$ do termo de troca exato. Essa fração é um resultado de teoria de perturbação que fornece os melhores resultados para energias de atomização de moléculas. ${ }^{33}$

O diferencial do funcional HSE06 é que sua construção contém uma separação entre componentes de longo alcance (LA) e curto alcance (CA), feita apenas no operador de Coulomb do termo de troca, isto é,

$$
\frac{1}{r}=\frac{\operatorname{erf}(\omega r)}{r}+\frac{\operatorname{erfc}(\omega r)}{r}
$$

onde $\operatorname{erf}(x)$ é a função erro e $\operatorname{erfc}(x)=1-\operatorname{erf}(x)$. O primeiro termo corresponde à componente de longo alcance, conforme o comportamento da função erro, pois $\operatorname{erf}(x)=0$ para $x=0$ e $\operatorname{erf}(x) \rightarrow 1$ para $x \rightarrow \infty$. Dessa forma, $\omega$ é o parâmetro que ajusta a separação entre longo alcance e curto alcance. Além disso, testes numéricos indicam que as contribuições dos termos de longo alcance de PBE e HF são pequenos e similares. ${ }^{32}$ Então, o funcional HSE06 é escrito da seguinte forma

$$
E_{x c}^{\mathrm{HSE} 06}=\frac{1}{4} E_{x}^{\mathrm{HF}, \mathrm{CA}}(\omega)+\frac{3}{4} E_{x}^{\mathrm{PBE}, \mathrm{CA}}(\omega)+E_{x}^{\mathrm{PBE}, \mathrm{LA}}(\omega)+E_{c}^{\mathrm{PBE}} .
$$

Esse funcional converge para PBE0 quando $\omega=0$ e para PBE quando $\omega \rightarrow \infty$. Testes realizados empregando diferentes valores de $\omega$ mostraram que o uso de $\omega=0.11 \mathrm{bohr}^{-1}$ fornece um bom compromisso entre exatidão dos resultados e eficiência computacional, ${ }^{34}$ correspondendo ao valor utilizado nesse trabalho de $0,206 \AA^{-1}$.

\subsubsection{Correções de van der Waals}

Os materiais $\mathrm{A}_{2} \mathrm{M}^{\mathrm{II}} \mathrm{M}_{3}^{\mathrm{IV}} \mathrm{Q}_{8}$ cristalizam em estruturas em camadas e portanto interações de van der Waals (vdW) podem ter um papel importante nas interações e na determinação das propriedades desses compostos. As interações de van der Waals se originam de flutuações de densidade de carga e são tipicamente mais fracas que as interações relacionadas à ligações iônicas ou covalentes, porém são importantes em algumas classes de materiais como sólidos moleculares, macromoléculas e cristais com estrutura em camadas. ${ }^{35}$ Cálculos DFT com funcionais de troca e correlação usuais (LDA, GGA) não fornecem uma descrição adequada das interações de van der Waals e portanto métodos vêm sendo 
desenvolvidos para adicionar correções a fim de aprimorar essa descrição. Várias dessas propostas se baseiam na consideração de que devido à pequena magnitude dessa correção, ela não tem efeito direto significativo sobre a densidade eletrônica. Assim, nessa abordagem a contribuição das interações de van der Waals é adicionada apenas através da soma de um novo termo à energia total do cálculo DFT convergido,

$$
E=E_{\mathrm{DFT}}+E_{\mathrm{vdW}}
$$

e as consequentes modificações no cálculo das forças. As diferentes metodologias desse tipo de correção de vdW se distinguem pela forma como $E_{\mathrm{vdW}}$ é calculada. Nesse trabalho foram usados dois métodos, que serão descritos em seguida: o método de Tkatchenko e Scheffler (TS) com blindagem auto-consistente (self-consistent screening, SCS), ${ }^{36-37}$ e o método D3 de Grimme. ${ }^{38}$

No método TS+SCS, $E_{\mathrm{vdW}}$ tem a forma de interações atômicas par a par com dependência $R^{-6}$,

$$
E_{\mathrm{vdW}}=-\sum_{I} \sum_{J<I} \frac{f_{a}\left(R_{I J}\right) C_{6}^{I J}}{R_{I J}^{6}},
$$

onde $R_{I J}=\left|\mathbf{R}_{I}-\mathbf{R}_{J}\right|$ e $f_{a}\left(R_{I J}\right)$ é uma função de amortecimento que tem a finalidade de reduzir a contribuição de termos com $R_{I J}$ pequeno,

$$
f_{a}\left(R_{I J}\right)=\frac{1}{1+e^{-d\left[R_{I J} /\left(s_{R} R_{0 I J}\right)-1\right]}}
$$

onde $d$ e $s_{R}$ são parâmetros ajustados para funcionais específicos (para PBE, $d=20$ e $\left.s_{R}=0.94\right)$, e $R_{0 i j}$ é a soma de raios atômicos, $R_{0 I J}=R_{0 I}+R_{0 J}$.

O ambiente químico de cada sistema é levado em conta para determinar os coeficientes $C_{6}^{I J}$. Isso é feito através da modificação das polarizabilidades atômicas $\left(\alpha_{I}\right)$ e dos coeficientes para átomos de mesma espécie química $\left(C_{6}^{I I}\right)$ partindo de valores de referência para átomos livres. Para isso, são utilizadas as seguintes expressões, que se baseiam na relação entre polarizabilidade atômica e volume,

$$
\begin{gathered}
\alpha_{I}^{\mathrm{TS}}=\beta_{I} \alpha_{I}^{\text {livre }}, \\
C_{6}^{I I, \mathrm{TS}}=\beta_{I}^{2} C_{6}^{I I, \text { livre }} .
\end{gathered}
$$

O fator $\beta_{I}$, que define a razão entre o volume efetivo do átomo no sistema e o volume do átomo livre, é obtido através da partição de Hirshfeld para a densidade eletrônica,

$$
\beta_{I}=\frac{\int r^{3} w_{I}(\mathbf{r}) n(\mathbf{r}) d \mathbf{r}}{\int r^{3} n_{I}^{\text {livre }}(\mathbf{r}) d \mathbf{r}},
$$

onde $\mathbf{r}$ tem a origem tomada no núcleo do átomo $I, n(\mathbf{r})$ é a densidade eletrônica total, $n_{I}^{\text {livre }}(\mathbf{r})$ é a densidade do átomo livre $I$ (calculada com DFT), e $w_{I}(\mathbf{r})$ é o peso da partição 
de Hirshfeld,

$$
w_{I}(\mathbf{r})=\frac{n_{I}^{\text {livre }}(\mathbf{r})}{\sum_{J} n_{J}^{\text {livre }}(\mathbf{r})},
$$

onde a soma percorre todos os átomos do sistema. Além dos efeitos do ambiente químico local, também são considerados os efeitos de interação de longo alcance entre flutuações de densidade eletrônica. Esses efeitos são incluídos através da solução da equação autoconsistente

$$
\alpha_{I}^{\mathrm{SCS}}(\omega)=\alpha_{I}(\omega)-\alpha_{I}(\omega) \sum_{J \neq I} \alpha_{J}^{\mathrm{SCS}}(\omega) \tau_{I J},
$$

onde $\tau_{I J}$ é um tensor que descreve as interações de dipolos e

$$
\alpha_{I}=\frac{\alpha_{I}^{\mathrm{TS}}}{1+\left(\omega / \omega_{I}\right)^{2}}
$$

onde $\omega_{I}$ define uma frequência característica, $\omega_{I}=4 C_{6}^{I I, \mathrm{TS}} / 3\left(\alpha_{I}^{\mathrm{TS}}\right)^{2}$. Então, os coeficientes $C_{6}^{I J}$ são obtidos pelas equações

$$
\begin{gathered}
C_{6}^{I I}=\frac{3}{\pi} \int_{0}^{\infty} \alpha_{I}^{\mathrm{SCS}}(\omega) \alpha_{I}^{\mathrm{SCS}}(\omega) d \omega, \\
C_{6}^{I J}=\frac{2 C_{6}^{I I} C_{6}^{J J}}{\left(\alpha_{J}^{\mathrm{SCS}} / \alpha_{I}^{\mathrm{SCS}}\right) C_{6}^{I I}+\left(\alpha_{I}^{\mathrm{SCS}} / \alpha_{J}^{\mathrm{SCS}}\right) C_{6}^{J J}},
\end{gathered}
$$

e os raios atômicos também são redefinidos com base nos volumes efetivos,

$$
R_{0 I}=\left(\frac{\alpha_{I}^{\mathrm{SCS}}}{\alpha_{I}^{\text {livre }}}\right)^{\frac{1}{3}} R_{0 I}^{\text {livre }}
$$

e com todos esses fatores calcula-se a correção de energia do método TS+SCS.

O método D3 de Grimme propõe uma correção para energia com termos de dois e três corpos,

$$
E_{\mathrm{vdW}}=E^{(2)}+E^{(3)} .
$$

O termo de dois corpos é dado por

$$
E^{(2)}=-\sum_{n=6,8,10, \ldots}\left[\sum_{I} \sum_{J<I} s_{n} \frac{f_{a, n}\left(R_{I J}\right) C_{n}^{I J}}{R_{I J}^{n}}\right]
$$

ou seja, a forma do termo correspondente a cada $n$ é similar ao termo usado no método TS+SCS, com uma modificação na dependência em $R_{I J}$. Testes mostraram que os termos $\operatorname{com} n>8$ levam à instabilidade do método e não melhoram significativamente os resultados dos sistemas mais comuns, ${ }^{38}$ portanto somente são mantidos os termos com $n=6$ e $n=8$. As funções de amortecimento para cada termo são

$$
f_{a, n}\left(R_{I J}\right)=\frac{1}{1+6\left(R_{I J} /\left(s_{r, n} R_{0}^{I J}\right)\right)^{-\alpha_{n}}} .
$$


Os parâmetros fixados são $s_{r, 8}=1, \alpha_{6}=14$ e $\alpha_{8}=16,{ }^{38}$ enquanto $s_{6}, s_{8}$ e $s_{r, 6}$ são ajustados dependendo do funcional utilizado (para PBE, $s_{6}=1, s_{8}=0.722$ e $s_{r, 6}=1.217$ ). ${ }^{38}$ Os raios de corte $R_{0}^{I J}$ são fixados para todos os pares de átomos entre $\mathrm{H}$ e $\mathrm{Pu}$. Nesse método, a dependência do ambiente químico nos coeficientes $C_{6}^{I J}$ é incluída de uma forma diferente em relação ao método TS+SCS. Valores de referência para os coeficientes foram determinados usando diversas moléculas distintas. Então, o valor de $C_{6}^{I J}$ é obtido usando uma média ponderada, em que o peso depende das diferenças entre números de coordenação fracionários $(N C)$ dos átomos no sistema de interesse e nas moléculas de referência. Esses $N C$ são determinados com base nas distâncias entre átomos e em valores de raio atômico. ${ }^{38} \mathrm{De}$ forma explícita, os coeficientes são determinados através da relação

$$
C_{6}^{I J}=\frac{\sum_{i=1}^{N_{I}} \sum_{j=1}^{N_{J}} C_{6, r e f}^{I J}\left(N C_{i}^{I}, N C_{j}^{J}\right) L_{i j}}{\sum_{i=1}^{N_{I}} \sum_{j=1}^{N_{J}} L_{i j}}
$$

em que $N_{I}$ e $N_{J}$ são os números de moléculas de referência para os átomos $I$ e $J$, respectivamente, e $C_{6, r e f}^{I J}\left(N C_{i}^{I}, N C_{j}^{J}\right)$ são valores de referência obtidos a partir das moléculas nas quais o átomo $I$ tem $N C=N C_{i}^{I}$ e o átomo $J$ tem $N C=N C_{j}^{J}$. O peso $L_{i j}$ é definido por

$$
L_{i j}=\exp \left\{-4\left[\left(N C^{I}-N C_{i}^{I}\right)^{2}+\left(N C^{J}-N C_{j}^{J}\right)^{2}\right]\right\}
$$

onde $N C^{I}$ e $N C^{J}$ correspondem aos números de coordenação para os átomos no sistema de interesse. Por fim, os coeficientes $C_{8}^{I J}$ são determinados a partir dos $C_{6}^{I J} \cdot{ }^{38}$

O termo de três corpos tem a seguinte forma,

$$
E^{(3)}=-\sum_{I} \sum_{J<I} \sum_{K<J} f_{a, 3}\left(\bar{R}_{I J K}\right) E^{I J K}
$$

em que a função de amortecimento tem forma similar às usadas no termo de dois corpos $\left(R_{I J K}\right.$ é a média geométrica de $R_{I J}, R_{J K}$ e $\left.R_{K I}\right)$ e o termo $E^{I J K}$ depende das distâncias entre os pares de átoms do trio $I J K$ e dos ângulos internos do triângulo formado por $\mathbf{R}_{I J}$, $\mathbf{R}_{J K}, \mathbf{R}_{K I}$. A adição de $E^{(3)}$ pode piorar os resultados de parâmetros de rede em sólidos, ${ }^{39}$ e além disso seu custo cresce mais rapidamente com o número de átomos que o dos demais termos. Portanto, esse termo não é utilizado na implementação do VASP.

Vemos portanto que além da diferença na expressão da energia, devido ao termo com $n=8$ no método D3, os dois métodos diferem também nas ideias usadas para determinar os coeficientes que são usados no cálculo da energia. Essas diferenças resultam em alguns contrastes entre os resultados dos dois métodos.

\subsection{Sistemas periódicos e ondas planas}

Todos os compostos estudados nesse trabalho possuem um ordenamento atômico na forma de estrutura cristalina. A distribuição espacial dos átomos em um cristal é 
caracterizada por uma rede de pontos que obedecem a seguinte condição: dado $\mathbf{r}$ um ponto da rede, qualquer outro ponto da rede $\mathbf{r}^{\prime}$ satisfaz $\mathbf{r}^{\prime}=\mathbf{r}+\mathbf{T}$, onde $\mathbf{T}=n_{1} \mathbf{a}_{1}+n_{2} \mathbf{a}_{2}+n_{3} \mathbf{a}_{3}$, com $n_{1}, n_{2}$ e $n_{3}$ inteiros. Os vetores de translação $\mathbf{a}_{1}, \mathbf{a}_{2}$ e $\mathbf{a}_{3}$ definem a rede. Além das translações $\mathbf{T}$, outras operações de simetria também podem mapear a rede nela mesma, como rotações. As condições de simetria em redes tridimensionais estabelecem restrições sobre os vetores de translação $\mathbf{a}_{i}$, originando 14 tipos de rede, que são conhecidas como redes de Bravais. ${ }^{40}$ Além da rede de Bravais, para definir completamente uma estrutura cristalina, é necessário especificar um conjunto de átomos associado a todos os pontos da rede, denominado base. A base é definida pelas espécies químicas de seus átomos e pelas posições de cada átomo em relação ao ponto da rede, $\boldsymbol{\rho}_{j}=x_{j} \mathbf{a}_{1}+y_{j} \mathbf{a}_{2}+z_{j} \mathbf{a}_{3}$, com $0 \leq x_{j}, y_{j}, z_{j} \leq 1$. Portanto, a estrutura cristalina é um arranjo periódico de átomos, formado pela repetição da base de acordo com as translações da rede de Bravais. Devido à sua periodicidade, os sólidos cristalinos são modelados nos cálculos computacionais através de condições periódicas de contorno. Define-se uma célula do cristal, em princípio a célula unitária, e a partir das translações da rede essa célula se propaga infinitamente, definindo um cristal ideal.

Um conceito importante no estudo de sólidos cristalinos é o da rede recíproca. A rede recíproca é definida pelo conjunto de vetores $\mathbf{k}$ que geram ondas planas com a mesma periodicidade da rede de Bravais, ou seja, $e^{i \mathbf{k} \cdot(\mathbf{r}+\mathbf{T})}=e^{i \mathbf{k} \cdot \mathbf{r}}$, para qualquer translação da rede $\mathbf{T}$. Essa condição, que pode ser reescrita da forma $e^{i \mathbf{k} \cdot \mathbf{T}}=1$, é satisfeita se $\mathbf{k} \cdot \mathbf{T}=2 \pi n$ ( $n$ inteiro). Escrevendo o vetor da forma $\mathbf{k}=m_{1} \mathbf{b}_{1}+m_{2} \mathbf{b}_{2}+m_{3} \mathbf{b}_{3}\left(m_{1}, m_{2}, m_{3}\right.$ inteiros), os vetores $\mathbf{b}_{i}$ que definem a rede recíproca são dados por

$$
\mathbf{b}_{1}=2 \pi \frac{\mathbf{a}_{2} \times \mathbf{a}_{3}}{\mathbf{a}_{1} \cdot\left(\mathbf{a}_{2} \times \mathbf{a}_{3}\right)}, \mathbf{b}_{1}=2 \pi \frac{\mathbf{a}_{3} \times \mathbf{a}_{1}}{\mathbf{a}_{1} \cdot\left(\mathbf{a}_{2} \times \mathbf{a}_{3}\right)}, \mathbf{b}_{1}=2 \pi \frac{\mathbf{a}_{1} \times \mathbf{a}_{2}}{\mathbf{a}_{1} \cdot\left(\mathbf{a}_{2} \times \mathbf{a}_{3}\right)},
$$

o que leva à satisfação da condição acima, pois $\mathbf{b}_{i} \cdot \mathbf{a}_{j}=2 \pi \delta_{i j}$. No espaço recíproco define-se a Primeira Zona de Brillouin (ZB) segundo a construção de Wigner-Seitz: com segmentos de reta ligando um ponto da rede aos seus vizinhos, toma-se para cada segmento um plano normal a ele e cruzando o seu ponto médio; o menor volume fechado dentre desses planos forma a célula de Wigner-Seitz, no caso do espaço recíproco a ZB.

A periodicidade do ordenamento atômico tem consequências importantes nas características de funções de onda dos elétrons da estrutura cristalina, visto que o potencial de interação entre um elétron e os átomos tem a mesma periodicidade da rede. O Teorema de Bloch diz que as soluções da equação de Schrödinger de um elétron sujeito a um potencial periódico, $V(\mathbf{r}+\mathbf{R})=V(\mathbf{r})$, podem ser escritas sob a forma de um produto entre uma onda plana e uma função com mesma periodicidade do potencial, ou seja,

$$
\psi_{\mathbf{k}}(\mathbf{r})=e^{i \mathbf{k} \cdot \mathbf{r}} u_{\mathbf{k}}(\mathbf{r}), u_{\mathbf{k}}(\mathbf{r}+\mathbf{R})=u_{\mathbf{k}}(\mathbf{r})
$$

onde o vetor $\mathbf{k}$ pode sempre ser escolhido dentro da ZB. ${ }^{40}$ Desse resultado, segue que a densidade eletrônica da solução também tem a periodicidade da rede, pois $\left|\psi_{\mathbf{k}}(\mathbf{r}+\mathbf{R})\right|^{2}=$ 
$\left|\psi_{\mathbf{k}}(\mathbf{r})\right|^{2}$. Essas propriedades podem ser aplicadas à solução das equações de Kohn-Sham em sólidos cristalinos, ou seja, os orbitais de Kohn-Sham devem ter a forma das funções do teorema de Bloch,

$$
\Phi_{n, \mathbf{k}}(\mathbf{r})=e^{i \mathbf{k} \cdot \mathbf{r}} u_{n, \mathbf{k}}(\mathbf{r})
$$

onde $u_{n, \mathbf{k}}(\mathbf{r})$ tem a periodicidade da rede. Tal periodicidade sugere uma expansão em ondas planas como uma forma adequada para a função, ou seja, sendo $\mathbf{G}$ os vetores da rede recíproca, temos

$$
u_{n, \mathbf{k}}(\mathbf{r})=\sum_{\mathbf{G}} c_{n, \mathbf{k}}(\mathbf{G}) e^{i \mathbf{G} \cdot \mathbf{r}} .
$$

Evidentemente, o número de vetores $\mathbf{G}$ incluídos na soma não pode ser infinito, e a soma é truncada usando o seguinte critério,

$$
\frac{1}{2}|\mathbf{k}+\mathbf{G}|^{2}<E_{c}
$$

ou seja, apenas ondas planas com energia cinética menor que $E_{c}$, chamada energia de corte, são incluídas na base. Aumentando o valor da energia de corte, a representação dos orbitais torna-se mais exata, mas o custo computacional também aumenta. Por isso, testes de convergência são geralmente realizados para assegurar que os resultados dos cálculos não sejam significativamente afetados pelo aumento de $E_{c}$, garantindo um compromisso entre custo computacional e precisão dos resultados. Portanto, a forma explícita dos orbitais de Kohn-Sham é

$$
\Phi_{n, \mathbf{k}}(\mathbf{r})=\sum_{\frac{|\mathbf{k}+\mathbf{G}|^{2}}{2}<E_{c}} c_{n, \mathbf{k}}(\mathbf{G}) e^{i(\mathbf{k}+\mathbf{G}) \cdot \mathbf{r}} .
$$

Devido à dependência do vetor $\mathbf{k}$, para o cálculo de várias propriedades mostra-se necessário levar em consideração a contribuição de todos os vetores da ZB, ou seja, devem ser realizadas integrações sobre a ZB, como no caso da densidade eletrônica,

$$
n(\mathbf{r})=\frac{1}{\Omega_{Z B}} \sum_{i} \int_{Z B}\left|\Phi_{i, \mathbf{k}}(\mathbf{r})\right|^{2} d \mathbf{k},
$$

onde $\Omega_{Z B}$ denota o volume da ZB e a soma em $i$ percorre orbitais ocupados. Para realizar as integrações numericamente, são utilizadas somas sobre uma malha de pontos $\mathbf{k}$ da ZB. Quanto mais densa for essa malha, mais bem representada será a ZB, o que leva à maior exatidão dos cálculos, no entanto com maior custo computacional. Novamente, a convergência dos resultados com relação à malha de pontos $\mathbf{k}$ utilizada é necessária. Como as dimensões da ZB dependem inversamente dos comprimentos dos vetores da rede no espaço real, os cálculos com células cristalinas maiores envolvem menores números de pontos $\mathbf{k}$.

\subsection{Método Projector Augmented-Wave}

Apesar das características descritas anteriormente, o uso de uma base de ondas planas também gera dificuldades na descrição de funções de onda dos elétrons em sólidos. 
Isso se origina do diferente comportamento das funções de onda para diferentes regiões do espaço. Nas regiões próximas aos núcleos, as funções têm rápidas oscilações, enquanto nas regiões mais afastadas elas são suaves. ${ }^{41}$ Portanto, uma descrição adequada dessas funções requer um enorme número de ondas planas, o que pode tornar esse método inviável. As primeiras ideias para superar essa dificuldade consistem em usar um tratamento diferente para estados de caroço e estados de valência. Essa distinção se baseia nas seguintes observações: as distribuições de densidade se concentram em diferentes regiões do espaço, sendo que os elétrons de caroço têm concentrações mais próximas do núcleo, e os elétrons de valência, mais externas; além disso, as escalas de energia dos dois tipos de estados também são distintas e os elétrons do caroço são pouco afetados pelo ambiente químico ou perturbações externas.

No método dos pseudopotenciais, para distâncias ao núcleo inferiores a um raio de corte, a função de onda verdadeira é substituída por uma função de onda suave, e a forma original é mantida apenas a partir do raio de corte. Então, são construídos potenciais (pseudopotenciais) para descrever as interações do sistema a partir dessas novas funções de onda. Uma outra estratégia, usada no método de onda plana aumentada (augmented plane wave, APW) consiste em usar funções do tipo orbitais atômicos para as regiões próximas dos núcleos, e ondas planas para a região mais distante dos núcleos, mantendo assim todas as informações das funções de onda verdadeiras.

O método PAW, proposto por Blöchl, ${ }^{41}$ reúne ideias dos métodos de pseudopotenciais e APW. Nele, define-se uma transformação linear para obter os orbitais verdadeiros a partir de novas funções, denominadas pseudofunções, que devem simplificar o tratamento numérico. A transformação linear atua somente nas regiões de aumento, que são esferas em torno de cada átomo, e portanto as pseudofunções coincidem com os orbitais verdadeiros na região de interstício, fora das regiões de aumento. Dessa forma, as regiões de aumento são análogas às regiões de caroço dos métodos de pseudopotenciais. Para o desenvolvimento do método, são introduzidas ondas parciais $\phi_{i}$, que são soluções da equação de Schrödinger para o átomo isolado, e são usadas para construir o orbital $\Psi$ dentro da região de aumento através de uma combinação linear. Para cada onda parcial define-se uma pseudo-onda parcial $\tilde{\phi}_{i}$, que deve ser igual à $\phi_{i}$ fora da região de aumento e suave dentro da região de aumento. Dessa forma, podemos relacionar $\Psi$ a um pseudo-orbital $\tilde{\Psi}$ através da relação

$$
\Psi=\tilde{\Psi}-\sum_{i} c_{i} \tilde{\phi}_{i}+\sum_{i} c_{i} \phi_{i}
$$

pois as pseudo-ondas parciais geram $\tilde{\Psi}$ através de uma combinação linear com os mesmos coeficientes da combinação linear que gera $\Psi$ a partir dos $\phi_{i}$, ou seja,

$$
\Psi=\sum_{i} c_{i} \phi_{i} \text { e } \tilde{\Psi}=\sum_{i} c_{i} \tilde{\phi}_{i}, \text { dentro das regiões de aumento, }
$$

e as funções coincidem na região de interstício. Os pseudo-orbtiais $\tilde{\Psi}$ são escritos como uma expansão em ondas planas, facilitada pela suavização nas regiões de aumento. Para 
determinar os coeficientes $c_{i}$ são introduzidas funções fixas $\tilde{p}_{i}$, denominadas de funções projetoras, uma para cada pseudo-onda parcial. Essas funções são escolhidas para satisfazerem a relação

$$
\sum_{i}\left|\phi_{i}\right\rangle\left\langle\tilde{p}_{i}\right|=1 \text {, dentro das regiões de aumento, }
$$

de tal forma que os coeficientes são dados por $c_{i}=\left\langle\tilde{p}_{i} \mid \tilde{\Psi}\right\rangle$. Portanto, a relação entre os orbitais é,

$$
\Psi=\tilde{\Psi}+\sum_{i}(\phi-\tilde{\phi})\left\langle\tilde{p}_{i} \mid \tilde{\Psi}\right\rangle
$$

ou seja, o operador que leva $\tilde{\Psi}$ a $\Psi$ é

$$
\tau=1+\sum_{i}(\phi-\tilde{\phi})\left\langle\tilde{p}_{i}\right|
$$

Para os estados de caroço $\Psi^{c}$ utiliza-se a aproximação de caroço congelado, que mantém os estados fixos, importados de átomos isolados. Então, eles são expressados da forma

$$
\Psi_{i}^{c}=\tilde{\Psi}_{i}^{c}+\phi_{i}^{c}-\tilde{\phi}_{i}^{c}
$$

e não são necessárias funções projetoras e os coeficientes dos termos da decomposição são iguais à 1 .

Os valores de observáveis devem ser obtidos pelos valores esperados a partir de pseudo-orbitais. Para um operador $A$, isso corresponde obter o valor esperado de $\tilde{A}$, ou seja,

$$
\langle A\rangle=\sum_{i}\left\langle\Psi_{i}|A| \Psi_{i}\right\rangle=\sum_{i}\left\langle\tilde{\Psi}_{i}|\tilde{A}| \tilde{\Psi}_{i}\right\rangle
$$

onde o operador $\tilde{A}$ é dado por

$$
\tilde{A}=\tau^{\dagger} A \tau=A+\sum_{i, j}\left|\tilde{p}_{i}\right\rangle\left\langle\phi_{i}|A| \phi_{j}\right\rangle\left\langle\tilde{p}_{j}\left|-\sum_{i, j}\right| \tilde{p}_{i}\right\rangle\left\langle\tilde{\phi}_{i}|A| \tilde{\phi}_{j}\right\rangle\left\langle\tilde{p}_{j}\right|
$$

Portanto, na solução das equações de Kohn-Sham com o método PAW, as quantidades variacionais são os pseudo-orbitais $\tilde{\Psi}$, expandidos em ondas planas. Os resultados são obtidos com o uso das funções projetoras $\tilde{p}_{i}$ e das ondas parciais $\phi_{i}$ e $\tilde{\phi}_{i}$, que formam um conjunto especificado para cada espécie química.

\subsection{Detalhes computacionais e testes de convergência}

Todos os cálculos de DFT desse trabalho foram realizados com o pacote computacional VASP, que está entre os códigos mais utilizados atualmente para simulação computacional de materiais. O VASP utiliza um conjunto base de ondas planas e o método PAW, e nele estão implementados vários funcionais de troca e correlação (LDA, GGAs, híbridos) e diversas correções de van der Waals com diferentes níveis de aproximação. Além disso, o código possibilita o cálculo de diversas propriedades, como forças sobre 
átomos, tensor de stress, densidade de estados eletrônicos e matriz dielétrica. Nessa seção são discutidos alguns parâmetros computacionais relevantes e testes de convergência para os cálculos com o VASP desse trabalho.

Conforme discutido, dois importantes parâmetros computacionais dos cálculos são a energia de corte e a malha de pontos $\mathbf{k}$ para integração na ZB, e portanto testes de convergência foram realizados a fim de determinar os mais adequados para serem usados. Os testes foram realizados através do cálculo de energia total e otimização estrutural dos sistemas, ou seja, energias totais e parâmetros estruturais de equilíbrio obtidos com diferentes parâmetros foram comparados a fim de avaliar a convergência. A otimização das estruturas cristalinas nesses cálculos e nas demais etapas do trabalho foi feita através da minimização das forças atômicas e tensor de stress.

As forças atômicas são definidas pela derivada da energia com respeito às posições iônicas,

$$
F_{I \alpha}=-\frac{d E}{d R_{I \alpha}} .
$$

A obtenção das forças no método PAW envolve particularidades que a diferenciam da expressão obtida a partir do teorema de Hellmann-Feynman, que descreve as forças nos íons apenas como a interação eletrostática clássica entre os íons e entre íons e a densidade de carga dos elétrons. ${ }^{42} \mathrm{Na}$ formulação do método PAW, a a expressão para a força leva em conta os efeitos que o deslocamentos dos íons têm sobre mudanças das regiões de aumento e funções projetoras. ${ }^{43} \mathrm{O}$ tensor de stress relaciona variações de energia à deslocamentos uniformes dos átomos, ou seja transformações das posições atômicas definidas por um tensor,

$$
R_{I \alpha} \rightarrow R_{I \alpha}+\sum_{\beta} \varepsilon_{\alpha \beta} R_{I \beta}
$$

cuja consequência nos cristais é a deformação das células cristalinas, por contração ou expansão dos vetores da base, por exemplo. O tensor de stress médio é dado por ${ }^{44}$

$$
\sigma_{\alpha \beta}=\frac{1}{\Omega} \frac{d E}{d \varepsilon_{\alpha \beta}},
$$

onde $\Omega$ é o volume da célula unitária. Como no caso das forças, as características do método PAW têm consequências na determinação da expressão do tensor de stress. $^{43}$

Na figura 1 e na figura 2, são apresentados testes de convergência para $\mathrm{K}_{2} \mathrm{ZnSn}_{3} \mathrm{~S}_{8}$ nas estruturas 2D-triclínica e 3D-cúbica, respectivamente. Devido às simetrias da rede, alguns pontos $\mathbf{k}$ da ZB são mapeados em outros pelas operações de simetria, e portanto são equivalentes. Dessa forma, o número de pontos $\mathbf{k}$ irredutíveis na ZB é o número de pontos não equivalentes. Nos gráficos é possível observar que a partir de certos valores o aumento do número de pontos $\mathbf{k}$ ou da energia de corte não afetam significativamente os resultados, o que permite escolher para os cálculos parâmetros que combinem custo computacional razoável e boa qualidade dos resultados. É importante destacar ainda que o 
código utilizado, VASP, sugere valores de energia de corte para cada conjunto de projetores dos elementos químicos, de forma que os testes foram realizados para valores maiores ou iguais aos sugeridos. Além disso, como as células unitárias das estruturas estudadas são grandes, não é necessário uma malha com um elevado número de pontos $\mathbf{k}$, devido ao tamanho reduzido da ZB.

(a)

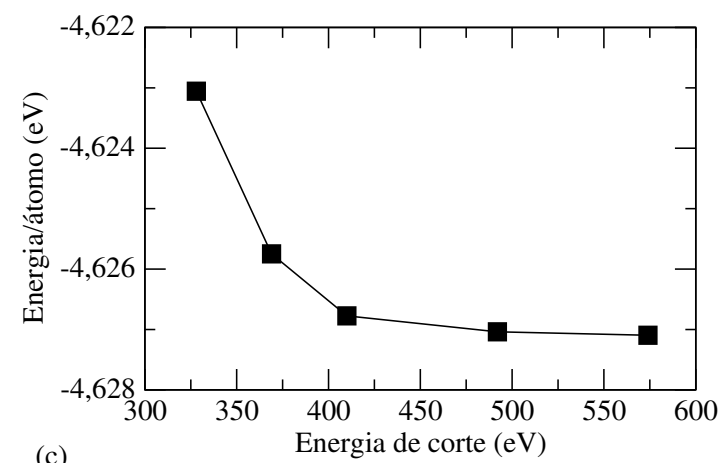

(c)

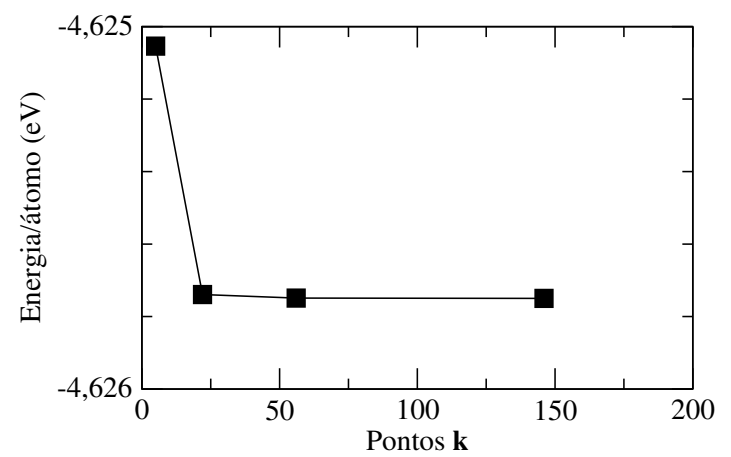

(b)
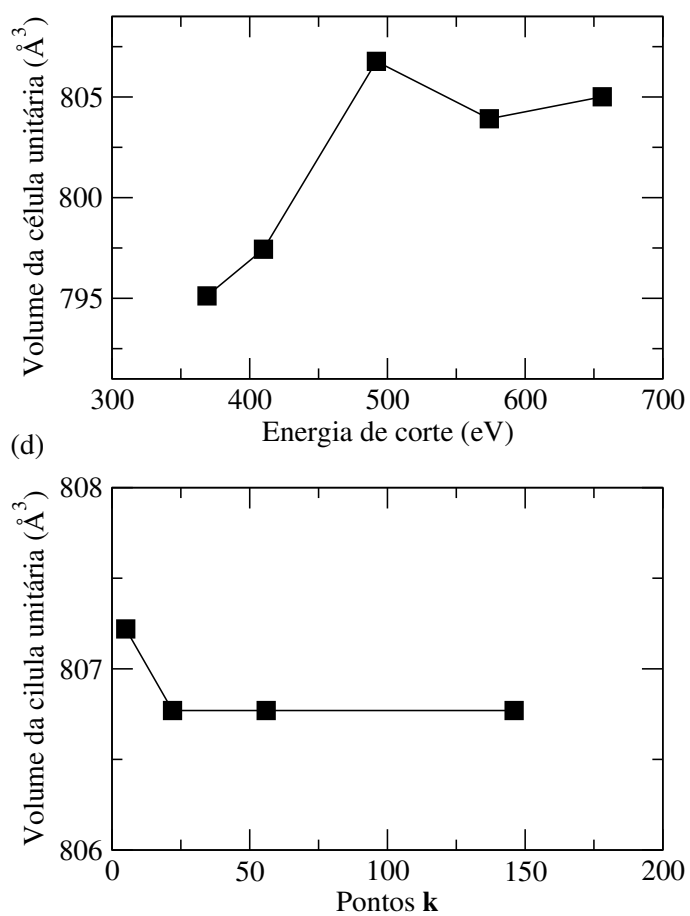

Figura 1 - Testes de convergência para a estrutura triclínica de $\mathrm{K}_{2} \mathrm{ZnSn}_{3} \mathrm{~S}_{8}$ em cálculos realizados com PBE+D3. (a) Energia por átomo da estrutura em função da energia de corte. (b) Volume da célula unitária em função da energia de corte. (c) Energia por átomo da estrutura em função do número de pontos $\mathbf{k}$ irredutíveis. (d) Volume da célula unitária em função do número de pontos $\mathbf{k}$ irredutíveis.

Fonte: Elaborada pelo autor.

Nesse trabalho foram utilizados para energia de corte os valores de $369 \mathrm{eV}$ para cálculos de energia total e de $492 \mathrm{eV}$ para os cálculos de otimização da estrutura. No entanto, como o VASP sugere um valor maior de energia de corte para o Li em comparação com os demais elementos químicos considerados, os cálculos com Li utilizaram energias de corte maiores, aumentando a energia sugerida pelo mesmo fator que o utilizado para obter os valores mencionados acima a partir da sugestão do VASP. Dessa forma, os cálculos com Li foram realizados com energias de corte de $488 \mathrm{eV}$ e $650 \mathrm{eV}$.

As malhas de pontos $\mathbf{k}$ adotadas nos cálculos dos compostos $\mathrm{Cs}_{2} \mathrm{M}^{\mathrm{II}} \mathrm{M}_{3}^{\mathrm{IV}} \mathrm{Q}_{8}$ (capítulo 3) mantiveram a mesma densidade de pontos em todas as estruturas. Dessa forma, em estruturas com células cristalinas maiores, e consequentemente ZB menores, são usados menos pontos $\mathbf{k}$. Por exemplo, a malha utilizada para $\mathrm{Cs}_{2} \mathrm{CdGe}_{3} \mathrm{~S}_{8}$ foi $3 \times 3 \times 1$ para 
(a)

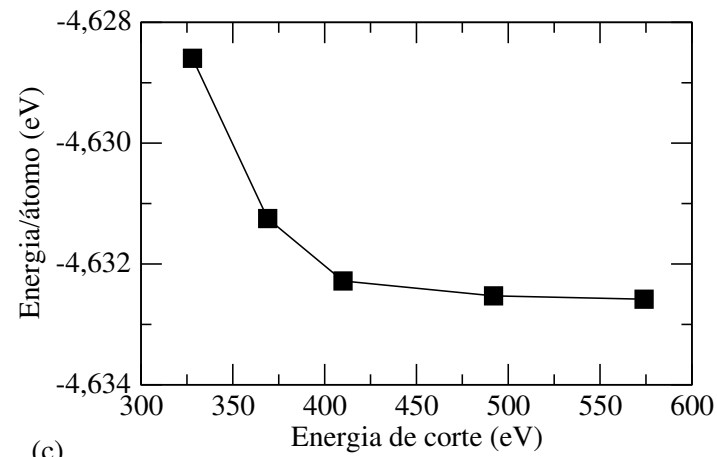

(c)

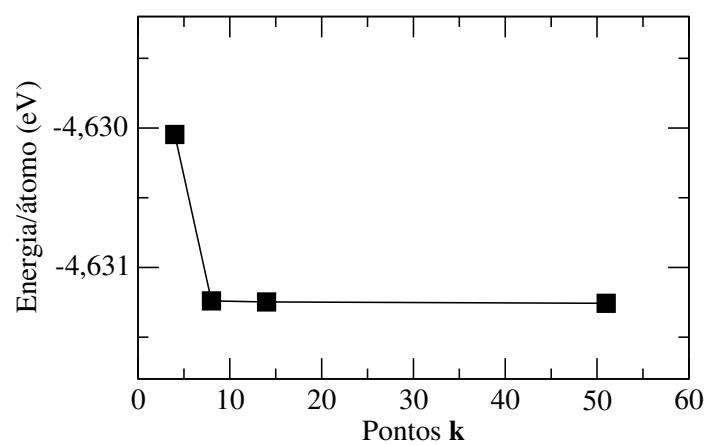

(b)

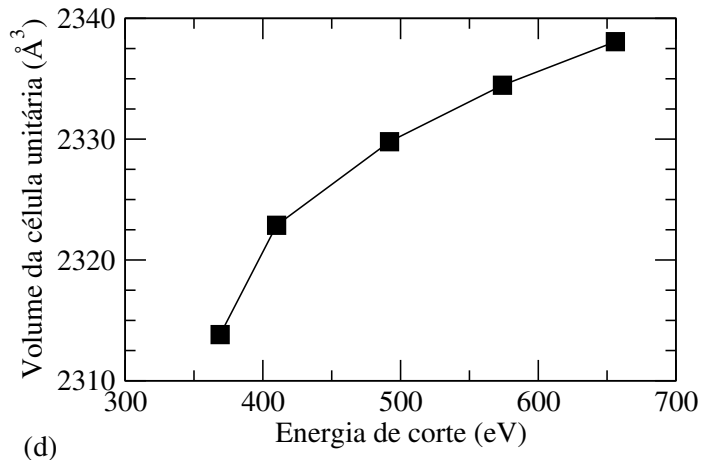

(d)

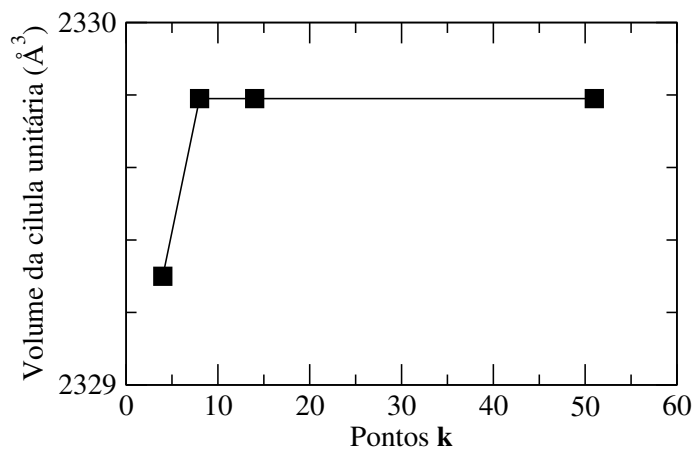

Figura 2 - Testes de convergência para a estrutura cúbica de $\mathrm{K}_{2} \mathrm{ZnSn}_{3} \mathrm{~S}_{8}$ em cálculos realizados com PBE+D3. (a) Energia por átomo da estrutura em função da energia de corte. (b) Volume da célula unitária em função da energia de corte. (c) Energia por átomo da estrutura em função do número de pontos $\mathbf{k}$ irredutíveis. (d) Volume da célula unitária em função do número de pontos $\mathbf{k}$ irredutíveis.

Fonte: Elaborada pelo autor.

cálculos de otimização estrutural e $5 \times 3 \times 2$ para os demais cálculos, como densidade de estados e propriedades ópticas, que requerem uma descrição mais refinada da ZB. Já para os cálculos para estudar estabilidade relativa das estruturas (capítulo 4), observou-se que a densidade de pontos $\mathbf{k}$ necesssária para obter uma descrição adequada de uma das estruturas (cúbica) era maior e por isso optou-se por aumentar a densidade de pontos em todas as estruturas, mantendo-a constante em todos os cálculos. Assim, a malha utilizada para $\mathrm{K}_{2} \mathrm{ZnSn}_{3} \mathrm{~S}_{8}$ na estrutura 2D-triclínica, por exemplo, foi $6 \times 6 \times 3$, com 56 pontos $\mathbf{k}$ irredutíveis na ZB. 


\section{PROPRIEDADES ESTRUTURAIS, ELETRÔNICAS E ÓPTICAS DOS CALCO- GENETOS QUATERNÁRIOS $\mathrm{Cs}_{2} \mathrm{M}^{\prime \prime} \mathrm{M}_{3}^{\mathrm{IV}} \mathbf{Q}_{8}$}

Devido à diversidade de band gaps medidos nos 11 compostos $\mathrm{Cs}_{2} \mathrm{M}^{\mathrm{II}} \mathrm{M}_{3}^{\mathrm{IV}} \mathrm{Q}_{8}$ sintetizados, essa família de materiais pode ser estudada para controle do band gap através de alterações na composição. Além disso, estender a caracterização para todas as 24 composições possíveis é importante para aprofundar o entendimento dos efeitos da alteração de $\mathrm{M}^{\mathrm{II}}, \mathrm{M}^{\mathrm{IV}}$ e $\mathrm{Q}$ nas propriedades desses materiais. Nesse capítulo, são apresentados resultados e discussões para as propriedades dos 24 calcogenetos $\mathrm{Cs}_{2} \mathrm{M}^{\mathrm{II}} \mathrm{M}_{3}^{\mathrm{IV}} \mathrm{Q}_{8}$, e os efeitos da composição química. Ele está dividido nas seções: (3.1) Estruturas cristalinas; (3.2) Parâmetros estruturais de equilíbrio; (3.3) Propriedades energéticas; (3.4) Propriedades eletrônicas; (3.5) Propriedades ópticas.

\subsection{Estruturas cristalinas}

Dos 11 compostos sintetizados da família $\mathrm{Cs}_{2} \mathrm{M}^{\mathrm{II}} \mathrm{M}_{3}^{\mathrm{IV}} \mathrm{Q}_{8}, 10$ cristalizam na estrutura ortorrômbica de grupo espacial $P 2_{1} 2_{1} 2_{1}$, com 4 fórmulas unitárias por célula unitária. ${ }^{20}$ A célula cristalina dessa estrutura é formada por três vetores ortogonais, definidos pelos parâmetros de rede diferentes $a, b$ e $c$. A estrutura desses compostos, que está representada na figura 3, é caracterizada pelo empilhamento de camadas de $\mathrm{M}^{\mathrm{II}} \mathrm{M}_{3}^{\mathrm{IV}} \mathrm{Q}_{8}$ ao longo da direção [010]. Em cada camada, os átomos de $\mathrm{M}^{\mathrm{II}}$ e $\mathrm{M}^{\mathrm{IV}}$ ocupam sítios tetraédricos, coordenados por 4 átomos de Q, enquanto os átomos de Cs se localizam embaixo e em cima de cada camada. ${ }^{20} \mathrm{O}$ único dos compostos que não adota essa estrutura, $\mathrm{Cs}_{2} \mathrm{ZnGe}_{3} \mathrm{~S}_{8}$, cristaliza em uma estrutura monoclínica, grupo espacial $P 2_{1} / n$, muito similar à estrutura anterior. A célula cristalina monoclínica se distingue da célula ortorrômbica porque nela dois dos vetores não são ortogonais entre si, sendo que no caso de $\mathrm{Cs}_{2} \mathrm{ZnGe}_{3} \mathrm{~S}_{8}$, o ângulo entre a e b é de $97,7^{\circ} \cdot{ }^{20}$ Devido a isso, o empilhamento das camadas é alterado em relação à estrutura ortorrômbica, sendo essa a principal diferença entre as estruturas.

\subsubsection{Comparação entre as estruturas ortorrômbica e monoclínica}

Para estudar todos os 24 compostos $\mathrm{Cs}_{2} \mathrm{M}^{\mathrm{II}} \mathrm{M}_{3}^{\mathrm{IV}} \mathrm{Q}_{8}$ é importante examinar a relevância das diferenças entre as estruturas ortorrômbica e monoclínica, a fim de avaliar as estruturas cristalinas a serem usadas para cada composto, principalmente para aqueles sem caracterização experimental. Para determinar o quanto importantes são essas diferenças e para analisar a estabilidade relativa entre as duas estruturas, foram realizados cálculos com ambas para os compostos $\mathrm{Cs}_{2} \mathrm{CdSn}_{3} \mathrm{Se}_{8}$ e $\mathrm{Cs}_{2} \mathrm{ZnGe}_{3} \mathrm{~S}_{8}$, com PBE e PBE+TS+SCS. Os parâmetros de rede de equilíbrio e energias relativas, são mostrados na tabela 1 .

Para cada funcional, as diferenças entre os parâmetros de rede das duas estruturas 


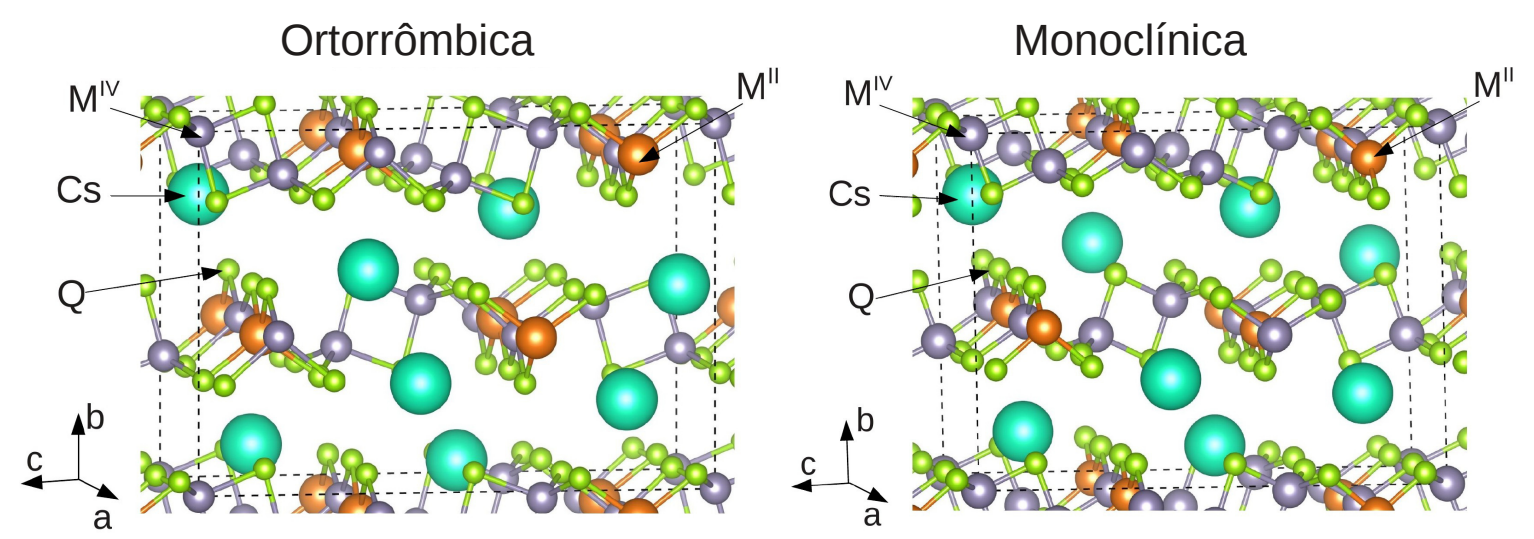

Figura 3 - Representação das estruturas cristalinas ortorrômbica e monoclínica dos compostos $\mathrm{Cs}_{2} \mathrm{M}^{\mathrm{II}} \mathrm{M}_{3}^{\mathrm{IV}} \mathrm{Q}_{8}$. Os átomos de $\mathrm{Cs}, \mathrm{M}^{\mathrm{II}}, \mathrm{M}^{\mathrm{IV}}$, Q são representados por esferas de cor ciano, laranja, roxo e verde, respectivamente.

Fonte: Elaborada pelo autor.

de um mesmo composto são menores que $3 \%$ e além disso o ângulo $\gamma$, que define o desvio da estrutura monoclínica para a ortorrômbica, difere de $90^{\circ}$ por valores que vão de $0,4^{\circ}$ a $3,3^{\circ}$. Dessa forma, há grandes semelhanças estruturais entre as duas estruturas. Além disso, elas têm energias muito próximas, pois as diferenças de energia entre as estruturas variam de $-0,5 \mathrm{meV} /$ átomo a $5,5 \mathrm{meV} /$ átomo. Portanto, devido à similaridade estrutural e energética entre as duas estruturas, optou-se por fazer o estudo dos 24 compostos $\mathrm{Cs}_{2} \mathrm{M}^{\mathrm{II}} \mathrm{M}_{3}^{\mathrm{IV}} \mathrm{Q}_{8}$ na estrutura ortorrômbica, o que permite simplificar a análise sem comprometer a qualidade dos resultados das propriedades desses materiais.

Tabela 1 - Parâmetros de rede de equilíbrio e energias relativas dos compostos $\mathrm{Cs}_{2} \mathrm{CdSn}_{3} \mathrm{Se}_{8}$ e $\mathrm{Cs}_{2} \mathrm{ZnGe}_{3} \mathrm{~S}_{8}$ calculados nas estruturas ortorrômbica e monoclínica.

\begin{tabular}{llllll}
\hline & \multicolumn{2}{c}{ PBE } & & \multicolumn{2}{c}{ PBE+TS+SCS } \\
\cline { 2 - 3 } $\mathrm{Cs}_{2} \mathrm{CdSn}_{3} \mathrm{Se}_{8}$ & Ortorrômbica & Monoclínica & & Ortorrômbica & Monoclínica \\
\hline$a_{0}(\AA)$ & 7,37 & 7,37 & & 7,21 & 7,20 \\
$b_{0}(\AA)$ & 13,52 & 13,60 & & 11,50 & 11,67 \\
$c_{0}(\AA)$ & 17,21 & 17,21 & & 16,91 & 16,92 \\
$\gamma$ & $90^{\circ}$ & $90,4^{\circ}$ & & $90^{\circ}$ & $91,7^{\circ}$ \\
Energia $(\mathrm{meV} /$ átomo $)$ & 0 & $-0,2$ & & 0 & 5,5 \\
\hline $\mathrm{Cs}_{2} \mathrm{ZnGe}_{3} \mathrm{~S}_{8}$ & Ortorrômbica & Monoclínica & Ortorrômbica & Monoclínica \\
\hline$a_{0}(\AA)$ & 8,23 & 8,21 & 7,85 & 7,75 \\
$b_{0}(\AA)$ & 13,63 & 13,99 & & 12,17 & 12,06 \\
$c_{0}(\AA)$ & 18,99 & 19,00 & & 18,29 & 18,42 \\
$\gamma$ & $90^{\circ}$ & $92,9^{\circ}$ & & $90^{\circ}$ & $93,3^{\circ}$ \\
Energia $(\mathrm{meV} /$ átomo $)$ & 0 & 2,4 & & 0 & $-0,5$ \\
\hline
\end{tabular}

Fonte: BESSE; SABINO; DA SILVA. ${ }^{45}$ 

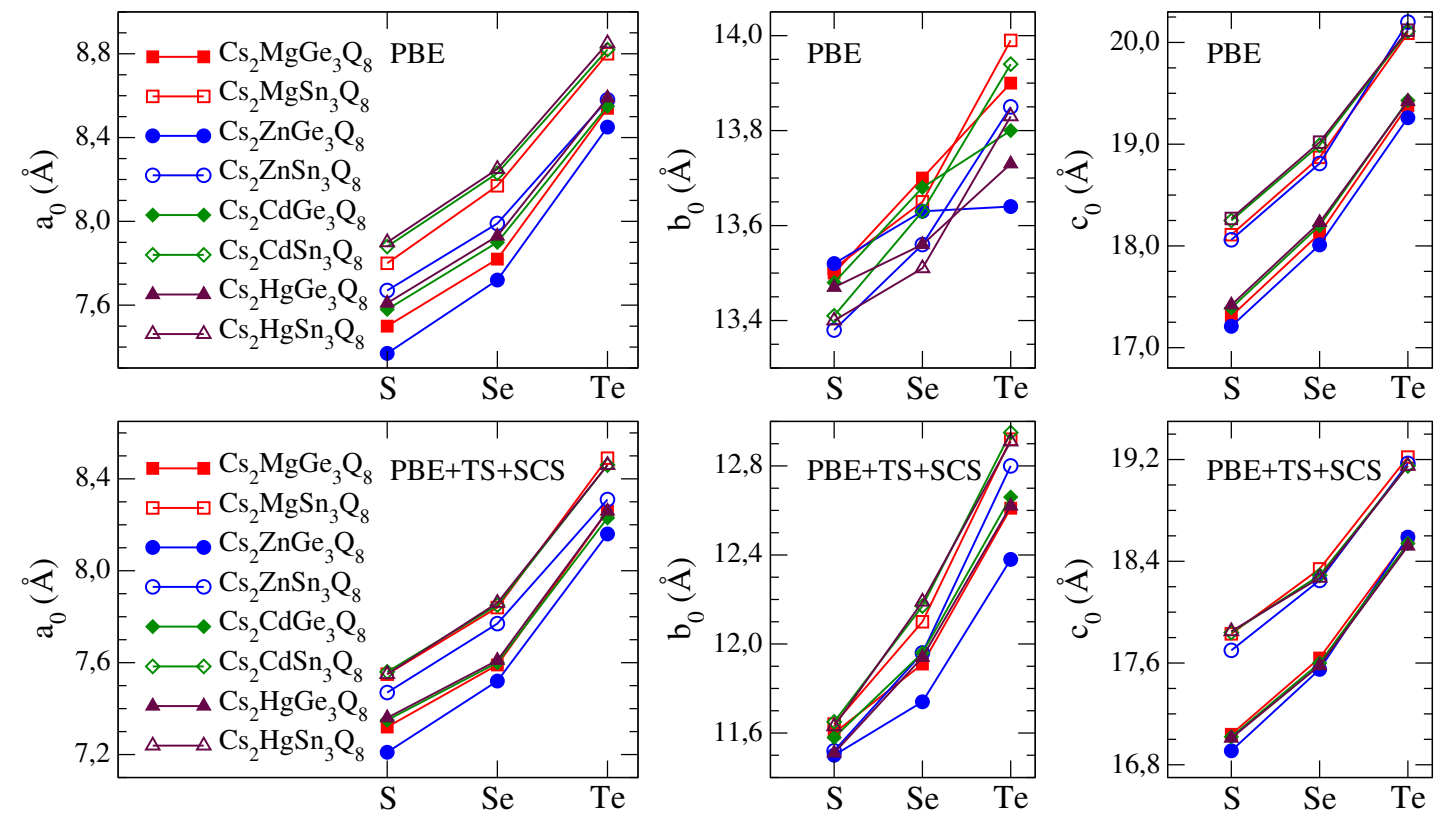

Figura 4 - Parâmetros de rede de equilíbrio, $a_{0}, b_{0}$ e $c_{0}$, da estrutura ortorrômbica dos 24 compostos $\mathrm{Cs}_{2} \mathrm{M}^{\mathrm{II}} \mathrm{M}_{3}^{\mathrm{IV}} \mathrm{Q}_{8}\left(\mathrm{M}^{\mathrm{II}}=\mathrm{Mg}, \mathrm{Zn}, \mathrm{Cd}, \mathrm{Hg} ; \mathrm{M}^{\mathrm{IV}}=\mathrm{Ge}, \mathrm{Sn} ; \mathrm{Q}=\mathrm{S}, \mathrm{Se}, \mathrm{Te}\right)$. Os gráficos da primeira linha correspondem aos resultados obtidos com o funcional PBE, e os da segunda linha foram obtidos com PBE+TS+SCS.

Fonte: Adaptada de BESSE; SABINO; DA SILVA. ${ }^{45}$

\subsection{Parâmetros estruturais de equilíbrio}

O primeiro passo para estudar as propriedades dos compostos $\mathrm{Cs}_{2} \mathrm{M}^{\mathrm{II}} \mathrm{M}_{3}^{\mathrm{IV}} \mathrm{Q}_{8}$, foi determinar as estruturas de equilíbrio dos 24 compostos. Mais especificamente, foram determinados os parâmetros de rede e posições atômicas de equilíbrio da estrutura ortorrômbica. Os parâmetros de equilíbrio foram calculados através da relaxação das estruturas, pela minimização das forças em todos os átomos e do tensor de stress. As estruturas foram relaxadas partindo das estruturas experimentais já disponíveis na literatura. ${ }^{20}$ Para os materiais sem dados experimentais, as estruturas iniciais foram obtidas substituindo os átomos das estruturas experimentais de acordo com as correspondentes mudanças de composição. Todas as estruturas foram otimizadas com PBE e PBE+TS+SCS.

Na figura 4 são mostrados os parâmetros de equilíbrio, $a_{0}, b_{0}$ e $c_{0}$, da estrutura ortorrômbica dos 24 compostos $\mathrm{Cs}_{2} \mathrm{M}^{\mathrm{II}} \mathrm{M}_{3}^{\mathrm{IV}} \mathrm{Q}_{8}$, calculados com PBE e PBE+TS+SCS, em função do calcogênio $(\mathrm{Q})$, pois a variação dessa espécie química gera as maiores mudanças nos parâmetros. Observa-se que conforme o número atômico de Q aumenta, com $\mathrm{M}^{\mathrm{II}}$ e $\mathrm{M}^{\mathrm{IV}}$ fixos, os parâmetros de rede também aumentam. Isso está de acordo com o esperado com base no conceito de raios atômicos, isto é, os parâmetros aumentam conforme aumentam os raios. Uma das formas de definir os raios atômicos de cada elemento é atribuir valores com base em distâncias interatômicas em vários compostos, o que permite predizer tendências de distâncias interatômicas e parâmetros de rede em sólidos ainda sem medidas. Aqui, o 
aumento dos parâmetros de rede pode ser previsto devido ao aumento dos raios, pois os

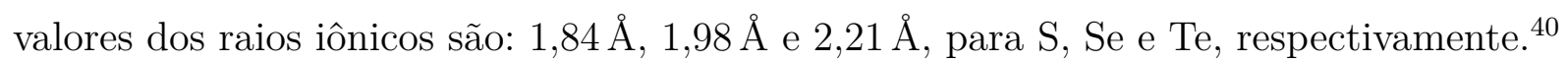
Uma tendência similar é observada com a variação dos elementos $\mathrm{M}^{\mathrm{IV}}$, isto é, os parâmetros de rede aumentam quando Ge é substituído por Sn, seguindo o aumento do raio iônico de $0,53 \AA$ para $0,71 \AA$.

Tabela 2 - Parâmetros de rede da estrutura ortorrômbica dos materiais $\mathrm{Cs}_{2} \mathrm{M}^{\mathrm{II}} \mathrm{M}_{3}^{\mathrm{IV}} \mathrm{Q}_{8}$, $\left(\mathrm{M}^{\mathrm{II}}=\right.$ $\mathrm{Mg}, \mathrm{Zn}, \mathrm{Cd}, \mathrm{Hg} ; \mathrm{M}^{\mathrm{IV}}=\mathrm{Ge}, \mathrm{Sn} ; \mathrm{Q}=\mathrm{S}, \mathrm{Se}, \mathrm{Te}$ ) obtidos com PBE (1) e PBE+TS+SCS (2), e valores experimentais para os materiais com dados disponíveis na literatura.

\begin{tabular}{|c|c|c|c|c|c|c|c|c|c|}
\hline \multirow[b]{2}{*}{ Composto } & \multicolumn{3}{|c|}{$\mathrm{a}_{0}(\AA)$} & \multicolumn{3}{|c|}{$b_{0}(\AA)$} & \multicolumn{3}{|c|}{$c_{0}(\AA)$} \\
\hline & 1 & 2 & Exp. & 1 & 2 & Exp. & 1 & 2 & ixp. \\
\hline $\mathrm{MorCe} \mathrm{S}$ & 50 & 32 & & 0 & 11,60 & & 1 & 17,04 & \\
\hline $\mathrm{Cs}_{2} \mathrm{MgGe}_{3} \mathrm{Se}_{8}$ & 7,82 & 7,59 & 7,66 & 13.70 & 11,91 & 12,72 & 8,12 & 17,64 & 17,77 \\
\hline $\mathrm{Cs}_{2} \mathrm{MgGe}_{3} \mathrm{Te}_{8}$ & 8,54 & 8,26 & & 13,90 & 12,61 & & & 18,58 & \\
\hline $\mathrm{Cs}_{2} \mathrm{MgSn}_{3} \mathrm{~S}_{8}$ & 7,80 & 7,55 & & 13,51 & 11,64 & & 8,11 & 17,83 & \\
\hline $\mathrm{Cs}_{2} \mathrm{MgSn}_{3} \mathrm{Se}_{8}$ & 8,17 & 7,84 & 7,91 & 13.65 & 12,10 & 12,88 & & 18,34 & 18,47 \\
\hline $\mathrm{Cs}_{2} \mathrm{MgSn}_{3} \mathrm{Te}_{8}$ & 8,80 & 8,39 & & 13,99 & 12,92 & & 20,09 & 19,22 & \\
\hline $\mathrm{Cs}_{2} \mathrm{Z}$ & 7,37 & 7,21 & 7,26 & & 11,50 & 12,62 & & & 16,98 \\
\hline $\mathrm{Cs}_{2} \mathrm{ZnGe}_{3} \mathrm{Se}_{8}$ & 7,72 & 7,52 & 7,58 & 1 & 11,74 & 12,62 & & 17,55 & 17,65 \\
\hline $\mathrm{Cs}_{2} \mathrm{ZnGe}_{3} \mathrm{Te}_{8}$ & 8,45 & 8,16 & 8,13 & & 12,38 & 13,11 & & 18,59 & 18,82 \\
\hline $\mathrm{Cs}_{2} \mathrm{Z}$ & 7,67 & 7,47 & & 13,38 & 11,52 & & & 17,70 & \\
\hline $\mathrm{Cs}_{2} \mathrm{ZnSn}_{3} \mathrm{Se}_{8}$ & 7,99 & 7,77 & 7,81 & 13.56 & 11,96 & 12,65 & 18,81 & 18,25 & 18,37 \\
\hline $\mathrm{Cs}_{2} \mathrm{Z}$ & 8,58 & 8,31 & & 13,85 & 12,80 & & 20 & 19,17 & \\
\hline $\mathrm{Cs}_{2} \mathrm{CdGe}_{3} \mathrm{~S}_{8}$ & $7-0$ & 7,35 & 7,42 & 13.48 & 11,58 & 12 , & & 17,02 & 17,08 \\
\hline $\mathrm{Cs}$ & 7,90 & 7,60 & 7,70 & & & 12,68 & & 17,60 & 17,82 \\
\hline $\mathrm{Cs}_{2} \mathrm{CdGe}_{3} \mathrm{Te}_{8}$ & 8,55 & 8,23 & & 13,80 & 12,66 & & & 18,54 & \\
\hline $\mathrm{Cs}_{2} \mathrm{C}$ & 7,88 & 7,56 & & 13,41 & 11,65 & & & 17,84 & \\
\hline $\mathrm{Cs}_{2} \mathrm{CdSn}_{3} \mathrm{Se}_{8}$ & 8,23 & 7,85 & 7,93 & 13.63 & 12,17 & 12,73 & 18,99 & 18,29 & 18,54 \\
\hline $\mathrm{Cs}_{2} \mathrm{CdSn}_{3} \mathrm{Te}_{8}$ & 8,82 & 8,46 & & 13,94 & 12,95 & & 20,11 & 19,15 & \\
\hline $\mathrm{Cs}_{2} \mathrm{H}$ & 7,61 & 7,36 & & & 11,51 & & & & \\
\hline $\mathrm{Cs}_{2} \mathrm{HgGe}_{3} \mathrm{Se}_{8}$ & 7,93 & 7,61 & & 13,56 & 11,94 & & 18,23 & 17,58 & \\
\hline $\mathrm{Cs}_{2} \mathrm{H}$ & 8,59 & 8,26 & & & 12,62 & & & 18,52 & \\
\hline $\mathrm{Cs}_{2} \mathrm{HgSn}_{3} \mathrm{~S}_{8}$ & 7,90 & 7,55 & 7,67 & 13.40 & 11,63 & 12,34 & 18,27 & 17,85 & 17,90 \\
\hline $\mathrm{Cs}_{2} \mathrm{HgSn}_{3} \mathrm{Se}_{8}$ & 8,25 & 7,86 & 7,92 & 13.51 & 12,19 & 12,67 & 19,02 & 18,27 & 18,54 \\
\hline $\mathrm{Cs}_{2} \mathrm{HgSn}_{3} \mathrm{Te}_{8}$ & 8,85 & 8,46 & & 13,83 & 12,91 & & 20,12 & 19,15 & \\
\hline
\end{tabular}

Fonte: MORRIS et al. ${ }^{20}$ BESSE; SABINO; DA SILVA. ${ }^{45}$

A comparação entre os resultados de PBE e PBE+TS+SCS mostra que não há mudanças nas tendências de variação dos parâmetros de rede e além disso os parâmetros diminuem com a inclusão das correções de vdW, o que se deve ao caráter atrativo dessas correções. A magnitude das diminuições no parâmetro $b_{0}$ (11, $2 \%$ em média) é maior que a das diminuições em $a_{0}$ (3, $6 \%$ em média) e $c_{0}$ (3, $4 \%$ em média). A diminuição do parâmetro $b_{0}$ tem correlação com a diminuição das distâncias entre as camadas, o que mostra que as correções de vdW afetam principalmente as interações entre camadas e portanto têm 
uma importante contribuição nessas interações, da mesma forma que em outros materiais com estruturas em camadas. ${ }^{35,46} \mathrm{Na}$ tabela 2 são apresentados os valores calculados dos parâmetros rede e também os resultados experimentais dos 11 compostos cujos dados estão disponíveis na literatura. ${ }^{20}$ As diferenças relativas dos parâmetros obtidos com PBE em relação aos dados experimentais têm médias de $2,8 \%$ para $a_{0}$ e $2,1 \%$ para $c_{0}$, dentro do esperado com o uso de PBE; com PBE+TS+SCS as diferenças relativas têm valores médios de $0,8 \%$ tanto para $a_{0}$ como para $c_{0}$, e portanto a inclusão de TS+SCS leva a melhores resultados. As diferenças relativas aos valores experimentais são mais significativas para o parâmetro $b_{0}$, sendo que com $\mathrm{PBE}$ os parâmetros são maiores por 7,1\% em média, enquanto os resultados de PBE+TS+SCS são menores que os experimentais por 6,0\% em média, ou seja, embora a inclusão de TS+SCS gere uma pequena melhora, os erros permanecem grandes. Esses resultados podem ser correlacionados com a descrição das interações entre as camadas, cujas distâncias estão ligadas à $b_{0}$ : a ligação entre as camadas é subestimada por PBE e superestimada por PBE+TS+SCS.

\subsubsection{Coordenação efetiva}

Uma ferramenta usada a fim de descrever as propriedades estruturais dos materiais, foi a aplicação do conceito de coordenação efetiva. ${ }^{47-48}$ Nesse método, o número de coordenação de cada átomo é determinado atribuindo a cada ligação um peso, que cresce conforme diminui o comprimento de ligação, definindo o número de coordenação efetiva (NCE). Para determinar o NCE de cada átomo, inicialmente são determinadas os comprimentos médios de ligação ponderados $d_{\text {med }}$ para cada átomo $i$,

$$
d_{\text {med }}^{i}=\frac{\sum_{j} d_{i j} \exp \left[1-\left(\frac{d_{i j}}{d_{\text {med }}^{i}}\right)^{6}\right]}{\sum_{j} \exp \left[1-\left(\frac{d_{i j}}{d_{\text {med }}^{i}}\right)^{6}\right]},
$$

onde $j$ percorre os demais átomos da estrutura e $d_{i j}$ é a distância entre os átomos $i$ e $j$. Com essa expressão, $d_{m e d}^{i}$ deve ser obtido de forma auto-consistente. Então, são determinados os NCE para cada átomo $i$, através da soma dos pesos atribuídos às ligações,

$$
\mathrm{NCE}_{i}=\sum_{j} \exp \left[1-\left(\frac{d_{i j}}{d_{m e d}^{i}}\right)^{6}\right] .
$$

Em comparação com a forma usual de atribuir o número de coordenação contando o número de vizinhos mais próximos, o conceito de coordenação efetiva tem a vantagem de não necessitar da definição arbitrária de uma distância de corte de ligação para cada estrutura. Isso é importante em estruturas complexas, onde existem diversos tipos de configurações geométricas e comprimentos de ligação distintos, como é o caso dos materiais $\mathrm{Cs}_{2} \mathrm{M}^{\mathrm{II}} \mathrm{M}_{3}^{\mathrm{IV}} \mathrm{Q}_{8}$. 
Os resultados da análise de coordenação efetiva aplicada às estruturas ortorrômbicas dos $\mathrm{Cs}_{2} \mathrm{M}^{\mathrm{II}} \mathrm{M}_{3}^{\mathrm{IV}} \mathrm{Q}_{8}$ são apresentados no Apêndice $\mathrm{A}$. Devido à proximidade entre valores de NCE e $d_{m e d}$ para átomos de um mesmo elemento químico em uma mesma estrutura, foram tomados valores médios para cada elemento químico em cada estrutura. Para $\mathrm{M}^{\mathrm{II}}$, o NCE é praticamente 4 em todas as estruturas (os maiores desvios são de 0,02), o que junto com a observação da estrutura cristalina mostra que as ligações $\mathrm{M}^{\mathrm{II}}-\mathrm{Q}_{4}$ se aproximam de tetraedros ideais. Já para $\mathrm{M}^{\mathrm{IV}}$, os valores de $\mathrm{NCE}$ variam de 3, 94 a 4,07, indicando que os tetraedros formados pelas ligações $\mathrm{M}^{\mathrm{IV}}-\mathrm{Q}_{4}$ são mais distorcidos, devido à variações de comprimentos de ligação. Os valores de NCE para Cs variam de 11, 44 a 13, 44 nas estruturas PBE e chegam a aumentar por cerca de 2 nas estruturas PBE+TS+SCS, devido à maior proximidade entre as camadas, o que diminui a distância dos átomos de Cs aos átomos de outra camada. Os valores de $d_{\text {med }}$ foram usados para atribuir raios atômicos aos elementos, com base no modelo de esferas rígidas, isto é, $R_{i}=d_{\text {med }}^{i} / 2$, onde para cada elemento foram tomadas médias sobre todas as estruturas. Esses resultados, mostrados na tabela 3, seguem as mesmas tendências dos raios iônicos mencionados anteriormente e portanto estão de acordo com a explicação das tendências de variação dos parâmetros de rede com a composição.

Tabela 3 - Raios atômicos baseados no modelo de esferas-rígidas e valores de $d_{m e d}$ dos elementos químicos presentes nos materiais $\mathrm{Cs}_{2} \mathrm{M}^{\mathrm{II}} \mathrm{M}_{3}^{\mathrm{IV}} \mathrm{Q}_{8}$, obtidos das estruturas ortorrômbicas otimizadas com $\mathrm{PBE}$ e $\mathrm{PBE}+\mathrm{TS}+\mathrm{SCS}$.

\begin{tabular}{ccc}
\hline Elemento químico & PBE & PBE+TS+SCS \\
\hline Cs & 2,06 & 2,02 \\
$\mathrm{Mg}$ & 1,32 & 1,30 \\
$\mathrm{Zn}$ & 1,26 & 1,25 \\
$\mathrm{Cd}$ & 1,36 & 1,34 \\
$\mathrm{Hg}$ & 1,37 & 1,36 \\
$\mathrm{Ge}$ & 1,22 & 1,21 \\
$\mathrm{Sn}$ & 1,30 & 1,30 \\
$\mathrm{~S}$ & 1,18 & 1,18 \\
$\mathrm{Se}$ & 1,25 & 1,25 \\
$\mathrm{Te}$ & 1,37 & 1,38 \\
\hline
\end{tabular}

Fonte: BESSE; SABINO; DA SILVA. ${ }^{45}$

\subsection{Propriedades energéticas}

Com as geometrias de equilíbrio dos calcogenetos $\mathrm{Cs}_{2} \mathrm{M}^{\mathrm{II}} \mathrm{M}_{3}^{\mathrm{IV}} \mathrm{Q}_{8}$ já determinadas, é possível estudar as propriedades energéticas desses materiais. Aqui, a discussão dessas propriedades é feita com base no estudo da energia de coesão e energia de ligação entre camadas nas estruturas. 


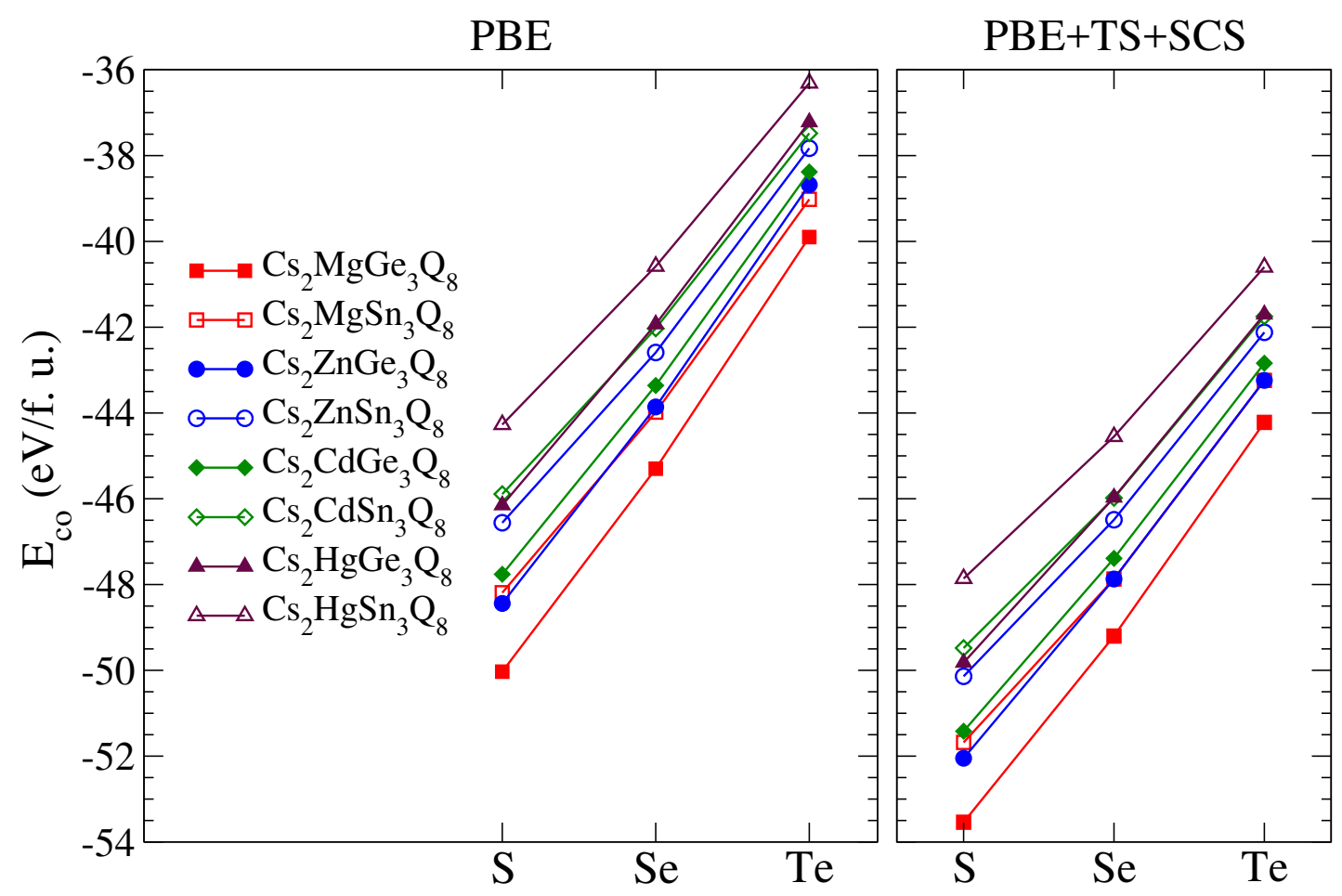

Figura 5 - Energia de coesão dos 24 compostos $\mathrm{Cs}_{2} \mathrm{M}^{\mathrm{II}} \mathrm{M}_{3}^{\mathrm{IV}} \mathrm{Q}_{8}\left(\mathrm{M}^{\mathrm{II}}=\mathrm{Mg}, \mathrm{Zn}, \mathrm{Cd}, \mathrm{Hg} ; \mathrm{M}^{\mathrm{IV}}=\right.$ $\mathrm{Ge}, \mathrm{Sn} ; \mathrm{Q}=\mathrm{S}, \mathrm{Se}, \mathrm{Te})$, obtidas com PBE e PBE+TS+SCS.

Fonte: Adaptada de BESSE; SABINO; DA SILVA. ${ }^{45}$

\subsubsection{Energia de coesão}

Para estudar a ligação e estabilidade dos calcogenetos $\mathrm{Cs}_{2} \mathrm{M}^{\mathrm{II}} \mathrm{M}_{3}^{\mathrm{IV}} \mathrm{Q}_{8}$, foi calculada a energia de coesão dos 24 compostos. A energia de coesão de um sólido é definida como a energia necessária para separar os átomos do cristal em átomos livres. Dessa forma, a energia de coesão por fórmula unitária (f.u.) é dada por

$$
E_{c o}=E_{f . u .}^{s o l}-\sum_{i} N_{i} E_{i}^{a t}
$$

onde $E_{f . u}^{s o l}$ é a energia do sólido por fórmula unitária, $N_{i}$ é o número de átomos de espécie química $i$ por fórmula unitária e $E_{i}^{a t}$ é a energia do átomo livre da espécie $i$ no estado fundamental. Como são utilizadas condições periódicas de contorno nos cálculos, para obter a energia dos átomos livres é necessário construir uma célula de grandes dimensões com apenas um átomo, para tornar desprezíveis as interações do átomo com suas imagens obtidas pelas translações da rede. Aqui, foi estabelecida uma distância mínima de $20 \AA$ entre os átomos.

Na figura 5 são mostradas as energias de coesão dos 24 compostos $\mathrm{Cs}_{2} \mathrm{M}^{\mathrm{II}} \mathrm{M}_{3}^{\mathrm{IV}} \mathrm{Q}_{8}$, obtidas com PBE e PBE+TS+SCS. Como a correção de vdW não afeta as energias dos átomos livres, a diferença entre os resultados de PBE e PBE+TS+SCS para um composto se origina totalmente da disparidade entre os valores de energia do sólido calculados com 
PBE e PBE+TS+SCS. Em todos os compostos essa diferença consiste essencialmente no aumento do módulo de $E_{c o}$ com adição de TS+SCS, devido ao caráter atrativo dessa correção, e as tendências de variação de $E_{c o}$ com a composição são as mesmas em PBE e PBE+TS+SCS. Os valores de $E_{c o}$ se tornam menos negativos a medida que o número atômico de Q aumenta, o que é consistente com o aumento observado nos parâmetros de rede. Essa mesma tendência é seguida para as mudanças de $\mathrm{M}^{\mathrm{II}}$ e $\mathrm{M}^{\mathrm{IV}}$. Para melhor entender o efeito da mudança de composição na energia de coesão através do papel das interações coulombianas, foi realizada uma análise das cargas efetivas nos átomos.

\subsubsection{Análise das cargas efetivas}

Em moléculas e sólidos, devido às alterações da distribuição eletrônica pela formação das ligações, não existem fronteiras bem definidas entre os átomos, particularmente entre qual parte da densidade eletrônica pode ser atribuída a um átomo ou outro. Portanto, para possibilitar uma análise das cargas dos átomos no cristal torna-se necessário usar alguma construção que permita distribuir a densidade eletrônica entre os átomos de forma sistemática. Aqui, foi utilizada a partição de Bader, ${ }^{49-50}$ na qual o espaço é dividido por superfícies de fluxo zero, isto é, em todos os pontos dessas superfícies o gradiente da densidade eletrônica é ortogonal à normal da superfície. Cada região delimitada pelas superfícies contém um núcleo atômico e portanto cada uma dessas regiões é atribuída a um átomo. A carga de Bader de cada átomo, $Q_{\mathrm{B}}^{i}$, é a integração da densidade eletrônica sobre o volume definido para o átomo. As cargas efetivas $Q_{\mathrm{ef}}^{i}$ em cada átomo foram obtidas por $Q_{\mathrm{ef}}^{i}=Z_{\mathrm{val}}^{i}-Q_{\mathrm{B}}^{i}$, onde $Z_{\mathrm{val}}$ é o número de elétrons do átomo $i$ na valência (9 para Cs; 12 para $\mathrm{Zn}, \mathrm{Cd}, \mathrm{Hg} ; 2$ para $\mathrm{Mg} ; 14$ para os $\mathrm{M}^{\mathrm{IV}}$ e 6 para $\left.\mathrm{Q}\right)$.

Os valores de $Q_{\text {ef }}$ dos átomos de uma mesma espécie química em um mesmo composto são muito similares e portanto a análise pode ser feita considerando valores médios de carga efetiva para cada espécie química do composto. Esses resultados são apresentados na figura 6 , e mostram a grande proximidade entre os resultados de PBE e PBE+TS+SCS; as pequenas diferenças são consequências das modificações na estrutura, pois a correção de vdW não afeta diretamente a distribuição eletrônica. Observa-se que os átomos de $\mathrm{Cs}, \mathrm{M}^{\mathrm{II}}$ e $\mathrm{M}^{\mathrm{IV}}$ doam elétrons para os átomos de $\mathrm{Q}$, o que está de acordo com o conceito de eletronegatividade (tabela 4), isto é, os átomos de maior eletronegatividade recebem a carga dos elétrons dos átomos menos eletronegativos. As tendências de variação da carga efetiva das espécies químicas devido às modificações na composição também se conformam ao esperado pela eletronegatividade dos elementos. O aumento na diferença da eletronegatividade dos elementos no composto leva a maiores valores absolutos de carga efetiva. Por exemplo, mantendo $\mathrm{M}^{\mathrm{II}}$ fixo, as magnitudes de carga diminuem com $\mathrm{Q}$ variando de $\mathrm{S}$ para Te, e mantendo $\mathrm{Q}$ fixo as cargas efetivas de $\mathrm{M}^{\mathrm{IV}}$ aumentam ao substituir Ge por Sn. 

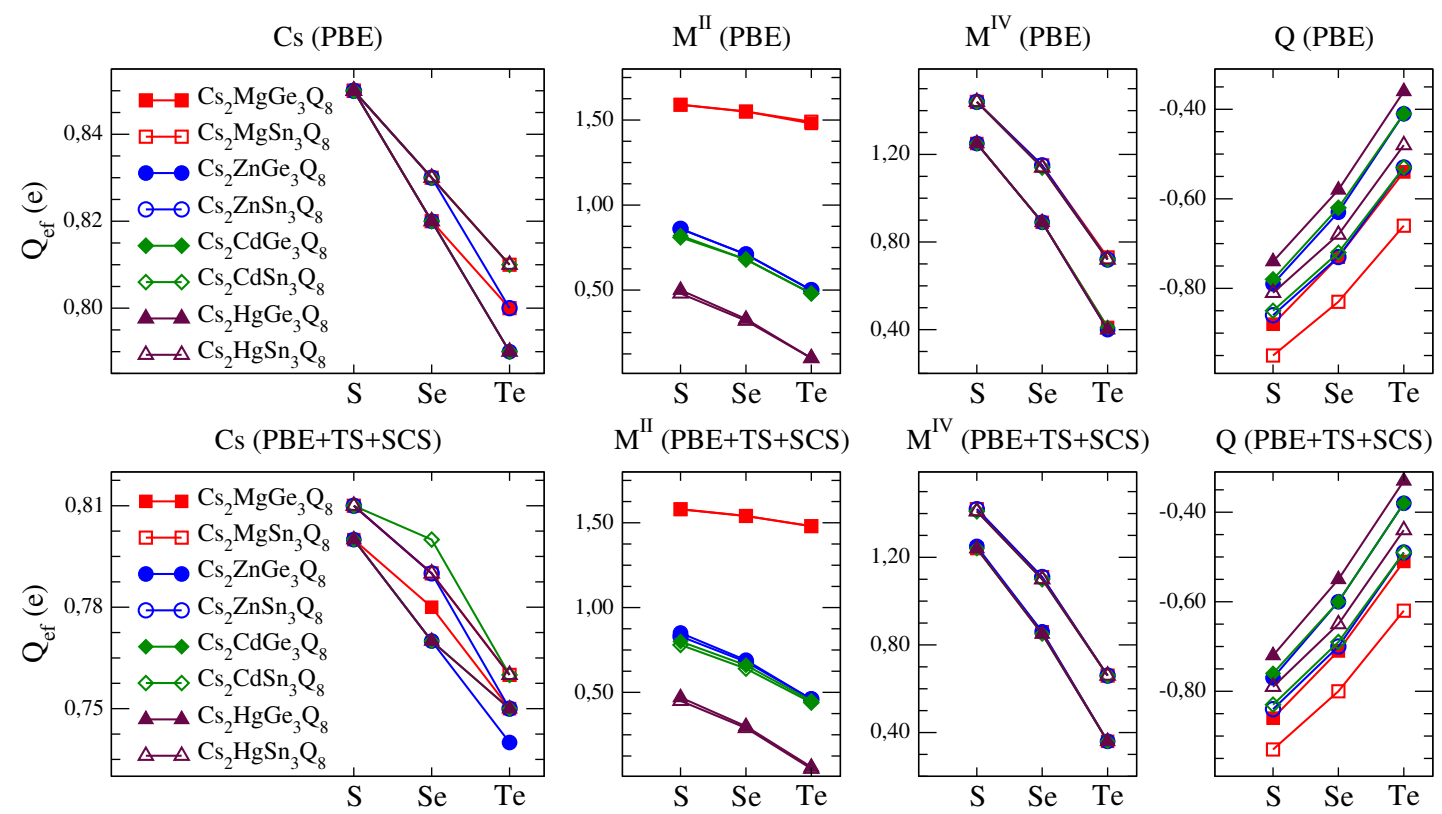

Figura 6 - Cargas efetivas médias de cada espécie química nos materiais $\mathrm{Cs}_{2} \mathrm{M}^{\mathrm{II}} \mathrm{M}_{3}^{\mathrm{IV}} \mathrm{Q}_{8}\left(\mathrm{M}^{\mathrm{II}}\right.$ $=\mathrm{Mg}, \mathrm{Zn}, \mathrm{Cd}, \mathrm{Hg} ; \mathrm{M}^{\mathrm{IV}}=\mathrm{Ge}, \mathrm{Sn} ; \mathrm{Q}=\mathrm{S}$, Se, Te). Os resultados da linha superior correspondem aos cálculos com PBE, e os da linha inferior foram obtidos com $\mathrm{PBE}+\mathrm{TS}+\mathrm{SCS}$.

Fonte: Adaptada de BESSE; SABINO; DA SILVA. ${ }^{45}$

Tabela 4 - Valores de eletronegatividade de Pauling dos elementos químicos presentes nos materiais $\mathrm{Cs}_{2} \mathrm{M}^{\mathrm{II}} \mathrm{M}_{3}^{\mathrm{IV}} \mathrm{Q}_{8}$.

\begin{tabular}{ccccccccccc}
\hline Elemento químico & $\mathrm{Cs}$ & $\mathrm{Mg}$ & $\mathrm{Zn}$ & $\mathrm{Cd}$ & $\mathrm{Hg}$ & $\mathrm{Ge}$ & $\mathrm{Sn}$ & $\mathrm{S}$ & $\mathrm{Se}$ & $\mathrm{Te}$ \\
\hline Eletronegatividade & 0,79 & 1,31 & 1,65 & 1,69 & 2,00 & 2,01 & 1,96 & 2,58 & 2,55 & 2,1 \\
\hline
\end{tabular}

Fonte: PAULING. ${ }^{51}$

A análise das cargas efetivas permite explicar as tendências da energia de coesão. Maiores magnitudes de cargas efetivas nos cátions e ânions, como as observadas ao diminuir o número atômico de Q, causam a intensificação das interações de Coulomb, que leva ao aumento da energia de coesão, conforme observado nos resultados apresentados.

\subsubsection{Energia de ligação entre camadas}

A determinação da energia de ligação entre camadas das estruturas cristalinas é importante para descrever as ligações que formam o sólido a partir do empilhamento das camadas, bem como para auxiliar no avanço da produção de estruturas bidimensionais através da remoção de camadas da estrutura. ${ }^{52}$ As energias de ligação entre camadas $\left(E_{b}\right)$ nos materiais $\mathrm{Cs}_{2} \mathrm{M}^{\mathrm{II}} \mathrm{M}_{3}^{\mathrm{IV}} \mathrm{Q}_{8}$ foram determinadas aqui através da diferença entre a energia da célula unitária por camada na estrutura original e a energia da célula unitária por camada numa estrutura modificada, em que as distâncias na direção de empilhamento (b) 
entre camadas sucessivas foi aumentada para no mínimo $12 \AA$, a fim de simular camadas isoladas.
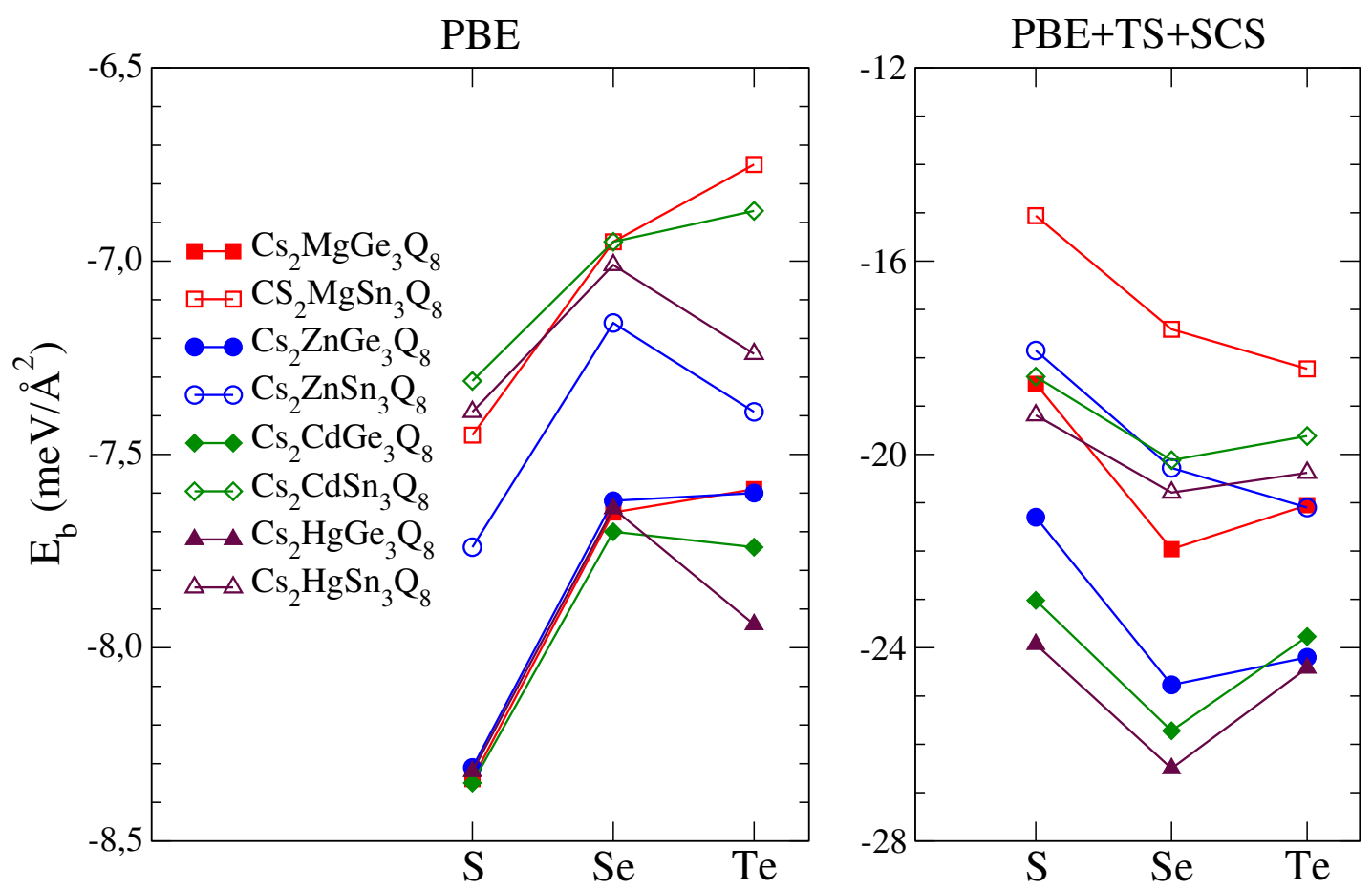

Figura 7 - Energias de ligação entre camadas dos 24 compostos $\mathrm{Cs}_{2} \mathrm{M}^{\mathrm{II}} \mathrm{M}_{3}^{\mathrm{IV}} \mathrm{Q}_{8}\left(\mathrm{M}^{\mathrm{II}}=\mathrm{Mg}, \mathrm{Zn}\right.$, $\left.\mathrm{Cd}, \mathrm{Hg} ; \mathrm{M}^{\mathrm{IV}}=\mathrm{Ge}, \mathrm{Sn} ; \mathrm{Q}=\mathrm{S}, \mathrm{Se}, \mathrm{Te}\right)$, obtidas com PBE e PBE+TS+SCS.

Fonte: Adaptada de BESSE; SABINO; DA SILVA. ${ }^{45}$

As energias de ligação dos 24 compostos $\mathrm{Cs}_{2} \mathrm{M}^{\mathrm{II}} \mathrm{M}_{3}^{\mathrm{IV}} \mathrm{Q}_{8}$ obtidas com PBE e PBE+ TS+SCS são mostradas na figura 7. Os valores são dados em unidades de energia/área, onde a área refere-se ao plano perpendicular à direção b, e nos cálculos corresponde ao produto dos parâmetros de rede $a_{0}$ e $b_{0}$. Observa-se que as energias obtidas com PBE+TS+SCS têm magnitude muito maior que as obtidas com PBE, com aumentos relativos próximos dos 200\%, o que evidencia novamente a importância da contribuição das correções de vdW nas interações entre as camadas desses materiais. Os valores obtidos com PBE+TS+SCS têm magnitudes entre 15 e $27 \mathrm{meV} / \AA^{2}$, bastante próximos dos valores obtidos por Björkman et al. ${ }^{52}$ de energias de ligação entre camadas. Esse trabalho estudou um conjunto de 86 compostos com estruturas em camadas usando diversas metodologias para incluir correções de vdW, como o método de Grimme e funcionais de correlação modificados para incluir efeitos de correlação não local, e obteve energias de ligação que em sua grande maioria estão entre 13 e $21 \mathrm{meV} / \AA^{2}{ }^{52}$ Além disso, valores experimentais recentes para o grafite, que é o material com mais estudos sobre a coesão entre camadas, variam entre 11,9 e $23 \mathrm{meV} / \AA^{2} \cdot{ }^{53-54}$ 


\subsection{Propriedades eletrônicas}

Para discutir as propriedades eletrônicas dos calcogenetos $\mathrm{Cs}_{2} \mathrm{M}^{\mathrm{II}} \mathrm{M}_{3}^{\mathrm{IV}} \mathrm{Q}_{8}$, é fundamental ter em conta as características gerais dos estados eletrônicos em sólidos cristalinos. Em átomos ou moléculas isolados, os estados eletrônicos têm uma distribuição discretizada na energia. Nos sólidos, com o aumento do número de átomos e suas interações, os níveis eletrônicos originam distribuições de estados que podem ser consideradas contínuas na energia, ou seja, formando bandas de energia. Dentre as bandas de energia, distinguem-se a banda de condução, que não tem estados ocupados a temperatura $0 \mathrm{~K}$, e a banda de valência, que contém os estados ocupados. A separação de energia entre a banda de valência e a banda de condução define o band gap $E_{g}$, presente nos isolantes e semicondutores e inexistente nos metais. Com a DFT é possível estudar as propriedades eletrônicas dos materiais, com base nos níveis de energia dos estados eletrônicos descritos pelos orbitais de Kohn-Sham. Nessa seção, as propriedades eletrônicas dos calcogenetos $\mathrm{Cs}_{2} \mathrm{M}^{\mathrm{II}} \mathrm{M}_{3}^{\mathrm{IV}} \mathrm{Q}_{8}$ são discutidas com base nos resultados de estrutura de bandas, densidade de estados e band gap desses materiais.

\subsubsection{Estrutura de bandas}

As bandas de energia em sólidos podem ser estudadas através de estruturas de bandas, que representam as relações de dispersão de energia em função do vetor de onda $\mathbf{k}$ da função de Bloch. Como mencionado anteriormente, os vetores $\mathbf{k}$ podem ser restringidos à ZB, e para representar as estruturas de bandas, são consideradas linhas entre pontos $\mathbf{k}$ de alta simetria da ZB. Na figura 8, é mostrada a ZB de uma rede ortorrômbica, com os pontos de alta simetria indicados, e também as linhas ao longo das quais foram calculadas as estruturas de bandas dos calcogenetos $\mathrm{Cs}_{2} \mathrm{M}^{\mathrm{II}} \mathrm{M}_{3}^{\mathrm{IV}} \mathrm{Q}_{8}$ nesse trabalho.

Com a estrutura de bandas de semicondutores é possível caracterizar o tipo de band gap, isto é, se ele é direto ou indireto. O band gap é direto se o máximo da banda de

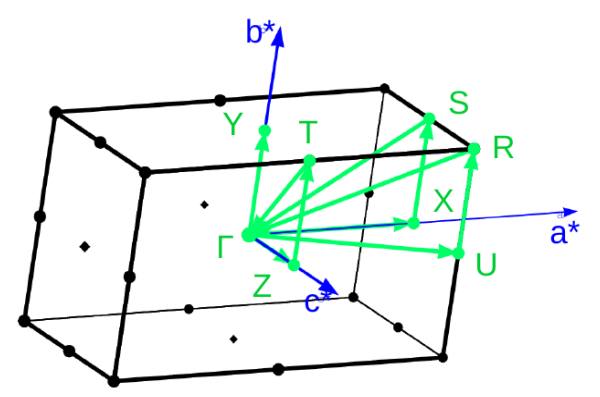

Figura 8 - Primeira Zona de Brillouin da rede ortorrômbica. Os pontos destacados e o caminho indicado correspondem às linhas usadas para o cálculo das estruturas de bandas dos compostos $\mathrm{Cs}_{2} \mathrm{M}^{\mathrm{II}} \mathrm{M}_{3}^{\mathrm{IV}} \mathrm{Q}_{8}$.

Fonte: Elaborada pelo autor. 


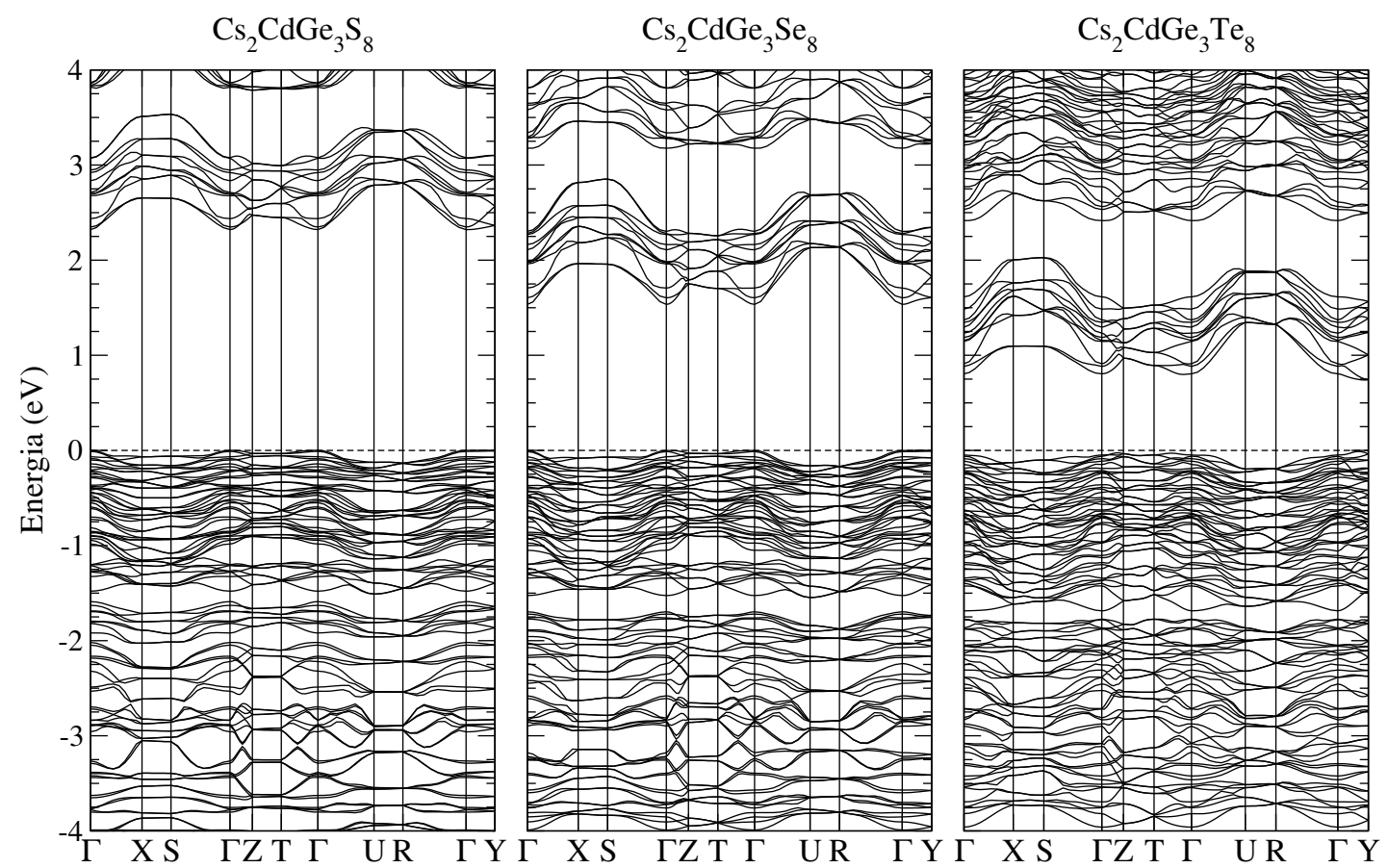

Figura 9 - Estruturas de bandas dos compostos $\mathrm{Cs}_{2} \mathrm{CdGe}_{3} \mathrm{Q}_{8}(\mathrm{Q}=\mathrm{S}$, Se, Te), calculadas com PBE. O topo da banda de valência está deslocado para energia zero.

Fonte: Adaptada de BESSE; SABINO; DA SILVA. ${ }^{45}$

valência (MBV) e o mínimo da banda de condução (MBC) estão localizados no mesmo ponto k, e é indireto se o MBV e o MBC estão em pontos k diferentes. Essa distinção é relevante, por exemplo, para processos ópticos, que ocorrem através de transições de elétrons entre as bandas com absorção ou emissão de fótons. Nesses processos, transições indiretas, que ocorrem para band gaps indiretos, devem envolver fônons para satisfazer a conservação de momento, diferentemente das transições diretas, relacionadas a band gaps diretos. ${ }^{40,55}$ Com a estrutura de bandas também é possível analisar a curvatura das bandas. Ela tem relação inversa com a massa efetiva dos elétrons, que mede a resposta do movimento dos elétrons na rede a campos eletromagnéticos aplicados.

As estruturas de bandas dos 24 compostos $\mathrm{Cs}_{2} \mathrm{M}^{\mathrm{II}} \mathrm{M}_{3}^{\mathrm{IV}} \mathrm{Q}_{8}$ foram calculadas com PBE. Nas figuras 9 e 10 são mostradas as estruturas de bandas dos compostos $\mathrm{Cs}_{2} \mathrm{CdGe}_{3} \mathrm{Q}_{8}$ e $\mathrm{Cs}_{2} \mathrm{ZnSn}_{3} \mathrm{Q}_{8}(\mathrm{Q}=\mathrm{S}, \mathrm{Se}, \mathrm{Te})$, respectivamente, e os demais resultados são mostrados no Apêndice A. Os resultados mostrados aqui exemplificam a semelhança entre as estruturas de bandas dos 24 compostos, que pode ser esperada pela similaridade das estruturas cristalinas e composições químicas, e demonstram suas características principais. Com esses resultados já é possível observar a existência de band gap nesses materiais, o intervalo de energia entre a última banda ocupada e a primeira vazia. As bandas de valência são achatadas e o MBV está localizado no ponto $\Gamma$, ou ao longo das linhas $\Gamma-Y, \Gamma-Z$ e $\Gamma-T$, sendo que nesses casos as diferenças de energia com relação ao ponto $\Gamma$ variam entre 10 e $35 \mathrm{meV}$. As bandas de condução são mais curvas e o MBC está no ponto $\Gamma$ para a maioria 

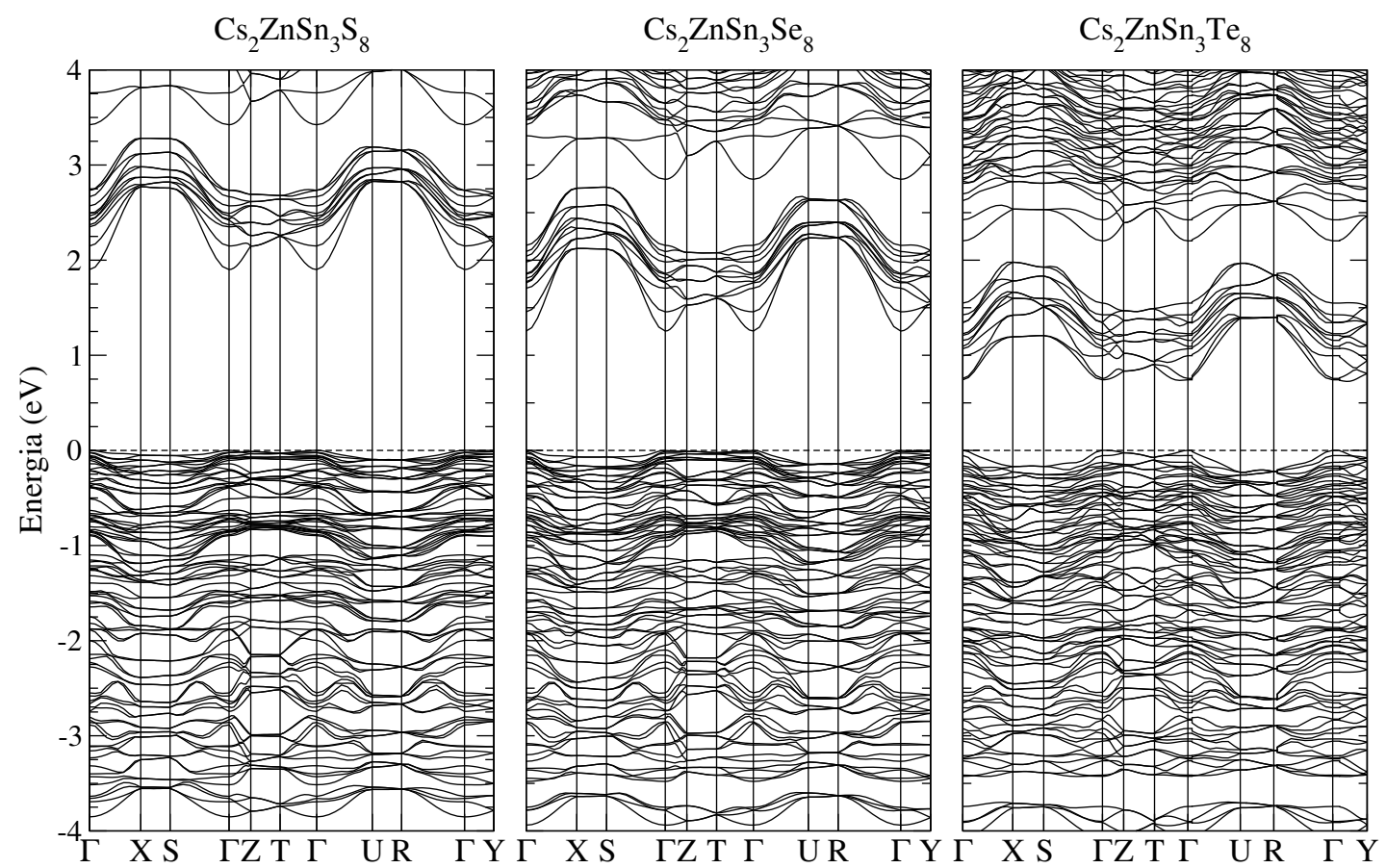

Figura 10 - Estruturas de bandas dos compostos $\mathrm{Cs}_{2} \mathrm{ZnSn}_{3} \mathrm{Q}_{8}(\mathrm{Q}=\mathrm{S}, \mathrm{Se}, \mathrm{Te})$, calculadas com PBE. O topo da banda de valência está deslocado para energia zero.

Fonte: Adaptada de BESSE; SABINO; DA SILVA. ${ }^{45}$

dos compostos. As exceções são $\mathrm{Cs}_{2} \mathrm{M}^{\mathrm{II}} \mathrm{Ge}_{3} \mathrm{Te}_{8}\left(\mathrm{M}^{\mathrm{II}}=\mathrm{Mg}, \mathrm{Zn}, \mathrm{Cd}, \mathrm{Hg}\right)$, para os quais o MBC está localizado ao longo da linha $\Gamma$-Y, com energias que são entre 25 e $70 \mathrm{meV}$ menores que a energia no ponto $\Gamma$. Portanto, a maioria dos compostos tem band gap direto no ponto $\Gamma$, sendo que nos demais as diferenças de energia entre o band gap indireto e o band gap direto no ponto $\Gamma$ são pequenas. Devido a isso, o valor do band gap direto no ponto $\Gamma$ pode ser usado como medida adequada do band gap nos calcogenetos $\mathrm{Cs}_{2} \mathrm{M}^{\mathrm{II}} \mathrm{M}_{3}^{\mathrm{IV}} \mathrm{Q}_{8}$. Além das modificações no band gap, a alteração da composição afeta também a curvatura das bandas. Observa-se que bandas de condução com menores energias tornam-se mais dispersivas ao substituir Ge por Sn, e também quando Q $=$ Se.

\subsubsection{Densidade de estados}

A densidade de estados (em inglês, density of states, DOS) mede o número de estados eletrônicos disponíveis em um intervalo de energia, e portanto fornece uma descrição de como os níveis eletrônicos estão distribuídos em função da energia no sistema. Além disso, é possível obter uma análise qualitativa da densidade de estados em termos do caráter da função de onda (em $s, p, d)$ para cada átomo. Essa análise é feita pela projeção dos orbitais de Kohn-Sham em regiões esféricas centradas nos átomos, e é apenas qualitativa porque é local, ou seja, como as projeções são feitas em regiões limitadas do espaço, elas não incluem completamente as contribuições de cada orbital. 
Foram calculadas as DOS dos 24 compostos $\mathrm{Cs}_{2} \mathrm{M}^{\mathrm{II}} \mathrm{M}_{3}^{\mathrm{IV}} \mathrm{Q}_{8}$ nas suas geometrias de equilíbrio. Nas figuras 11 e 12 são mostradas DOS dos compostos $\mathrm{Cs}_{2} \mathrm{CdGe}_{3} \mathrm{Q}_{8}$ e $\mathrm{Cs}_{2} \mathrm{ZnSn}_{3} \mathrm{Q}_{8}(\mathrm{Q}=\mathrm{S}$, Se, Te), respectivamente. Como no caso das estruturas de bandas, as características de DOS dos 6 compostos mostrados são bastante similares e o mesmo se verifica nos demais compostos $\mathrm{Cs}_{2} \mathrm{M}^{\mathrm{II}} \mathrm{M}_{3}^{\mathrm{IV}} \mathrm{Q}_{8}$, cujos resultados são apresentados no Apêndice A. As contribuições mais importantes nos níveis ocupados são dos estados $p$ originados de Q, que essencialmente compõem o topo da banda de valência. Também são importantes, em energias menores, as contribuições de estados $p$ originados de Cs e dos estados $d$ de $\mathrm{M}^{\mathrm{II}}$, exceto para $\mathrm{M}^{\mathrm{II}}=\mathrm{Mg}$. O fundo da banda de condução é predominantemente composto pelos estados $p$ de $\mathrm{Q}$ e $s$ de $\mathrm{M}^{\mathrm{IV}}$. Das diferenças mais importantes entre as DOS de compostos distintos pode ser destacada a variação do band gap.

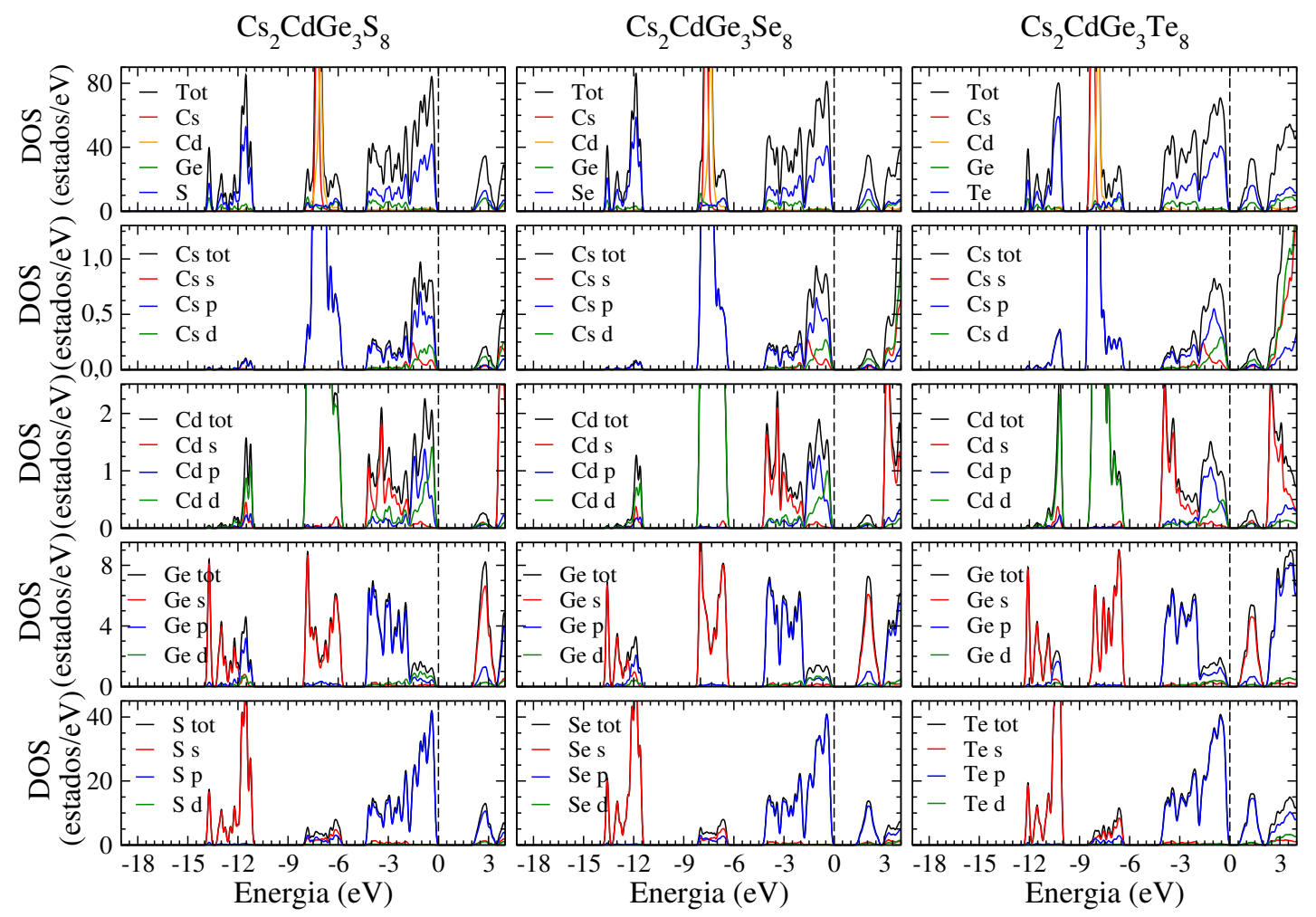

Figura 11 - Densidades de estados por célula cristalina dos compostos $\mathrm{Cs}_{2} \mathrm{CdGe}_{3} \mathrm{Q}_{8}(\mathrm{Q}=\mathrm{S}, \mathrm{Se}$, $\mathrm{Te}$ ), calculadas com PBE. O topo da banda de valência está deslocado para energia zero.

Fonte: Adaptada de BESSE; SABINO; DA SILVA. ${ }^{45}$

A inclusão de TS+SCS no cálculo não influencia diretamente os níveis eletrônicos, que são afetados indiretamente devido às alterações estruturais. Um exemplo desse efeito é mostrado na figura 13, para o composto $\mathrm{Cs}_{2} \mathrm{CdGe}_{3} \mathrm{~S}_{8}$. Observa-se que com PBE+TS+SCS, os estados estão mais espalhados em comparação com PBE, o que se deve à redução das distâncias interatômicas, principalmente entre camadas. 


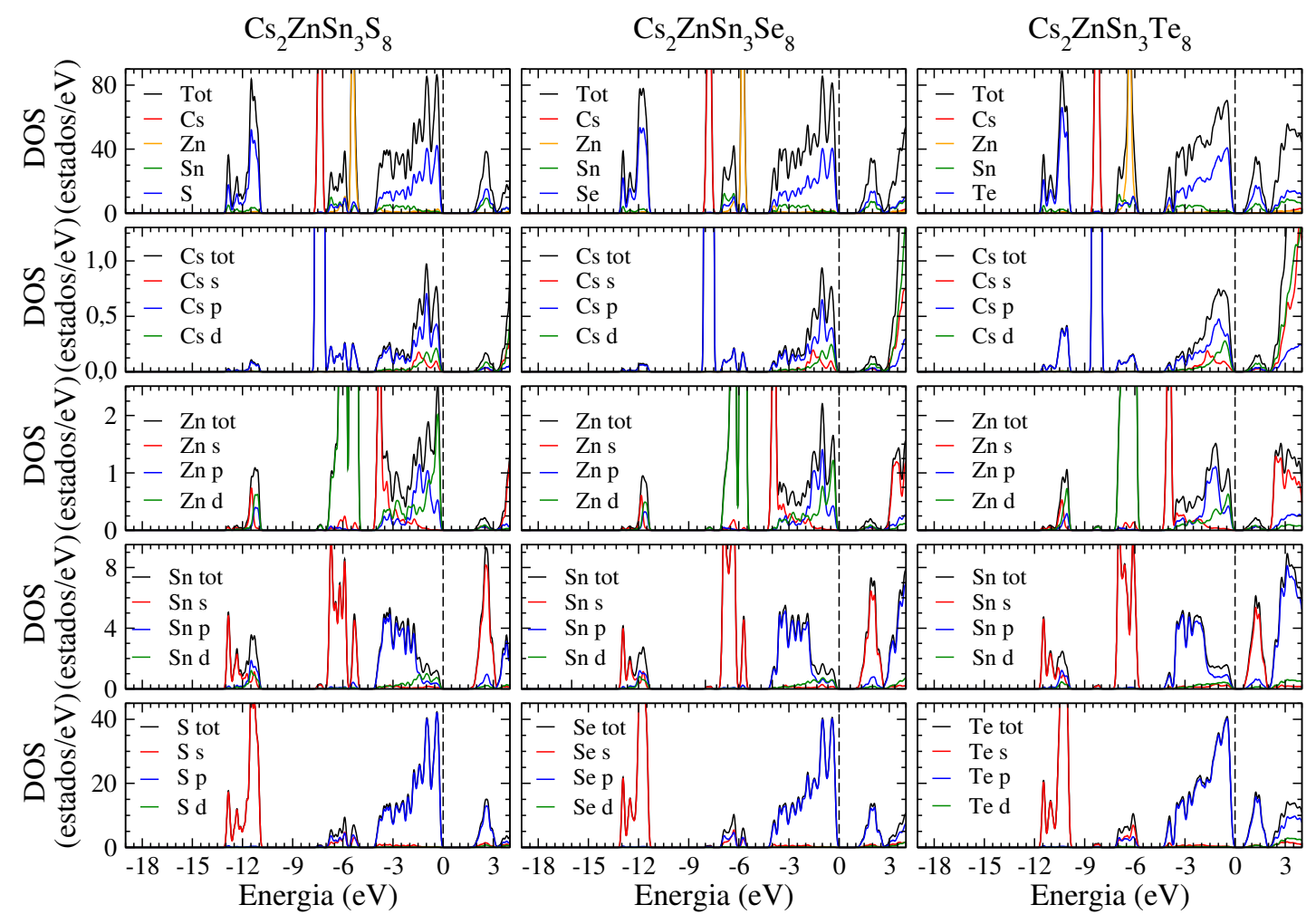

Figura 12 - Densidades de estados por célula cristalina dos compostos $\mathrm{Cs}_{2} \mathrm{ZnSn}_{3} \mathrm{Q}_{8}(\mathrm{Q}=\mathrm{S}$, Se, Te), calculadas com PBE. O topo da banda de valência está deslocado para energia zero.

Fonte: Adaptada de BESSE; SABINO; DA SILVA. ${ }^{45}$

\subsubsection{Band gaps}

Os resultados experimentais de 11 compostos $\mathrm{Cs}_{2} \mathrm{M}^{\mathrm{II}} \mathrm{M}_{3}^{\mathrm{IV}} \mathrm{Q}_{8}{ }^{20}$ indicaram uma grande diversidade de band gaps, a saber, valores entre 1,07 a 3,3 eV. Portanto, é importante estender essa caracterização do band gap para todos os 24 calcogenetos $\mathrm{Cs}_{2} \mathrm{M}^{\mathrm{II}} \mathrm{M}_{3}^{\mathrm{IV}} \mathrm{Q}_{8}$. Para calcular os band gaps, foram considerados os valores correspondentes ao ponto $\Gamma$, e as geometrias de equilíbrio determinadas com PBE e PBE+TS+SCS. Conforme discutido anteriormente, para obter valores mais adequados de band gap, além do funcional PBE, também foi utilizado o funcional híbrido HSE06. Todos esses resultados são mostrados na figura 14.

Em comparação com os dados experimentais, ${ }^{20}$ como esperado, os resultados de PBE subestimam o band gap, em aproximadamente $25 \%$ a $53 \%$. Essa tendência, que também já foi observada em cálculos com LDA para alguns dos compostos,${ }^{20}$ é mantida nas estruturas de PBE+TS+SCS, pois os band gaps são apenas levemente modificados pela inclusão de TS+SCS. Os resultados de HSE06 mostram melhor concordância com os dados experimentais, isto é, e os erros relativos médios são de 7,5\% e 14,3\% usando estruturas de PBE e PBE+TS+SCS, respectivamente. No entanto, observa-se que as tendências de variação do band gap devido às mudanças de composição são essencialmente 


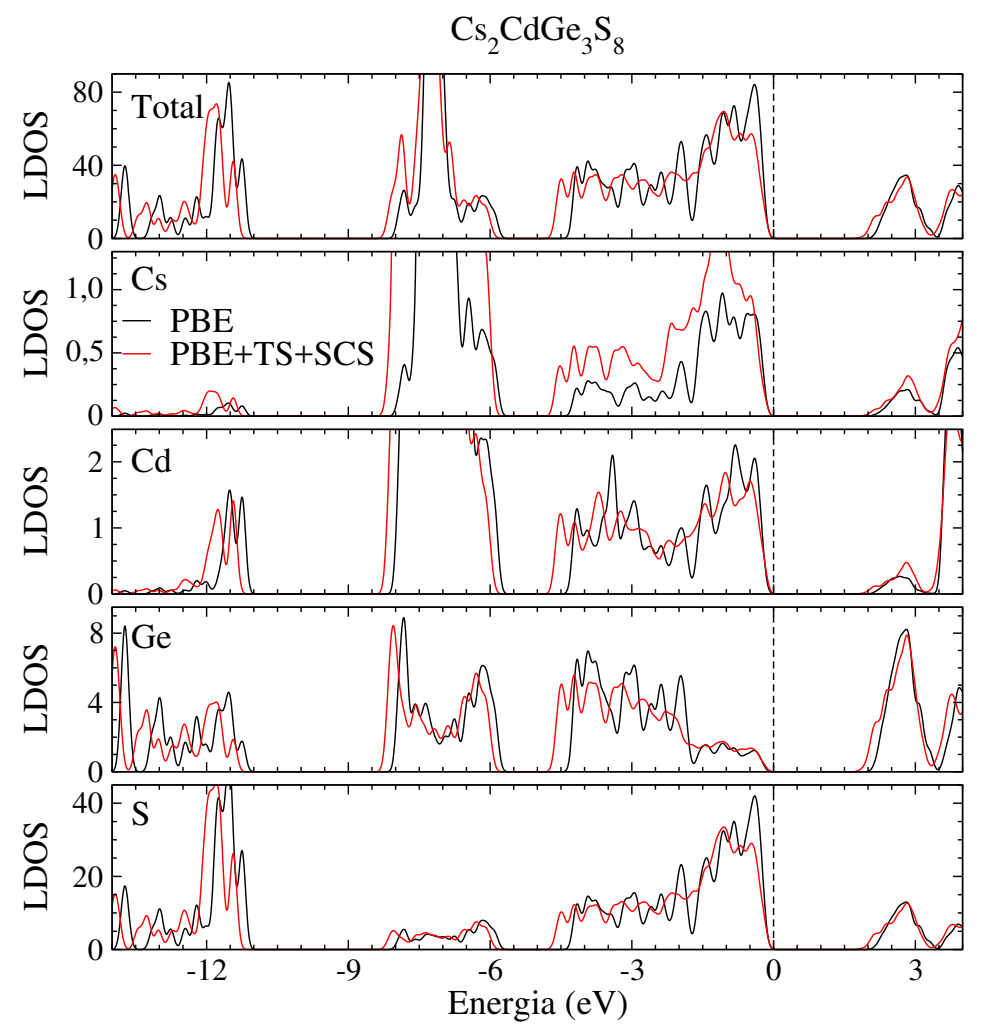

Figura 13 - Comparação entre as DOS do composto $\mathrm{Cs}_{2} \mathrm{CdGe}_{3} \mathrm{~S}_{8}$ obtidas com PBE e com PBE+TS+SCS. O topo da banda de valência foi colocado na energia zero.

Fonte: Adaptada de BESSE; SABINO; DA SILVA. ${ }^{45}$

mantidas nos quatro gráficos da figura 14. Em todos eles, o band gap diminui de forma aproximadamente linear com o aumento do volume da célula cristalina, e além disso a grande diversidade de band gaps entre esses materiais é demonstrada nos quatro casos, com valores que variam entre 1,35 e 3,49 eV para os cálculos de HSE06 nas estruturas de $\mathrm{PBE}$, por exemplo. Isso mostra que a composição química têm um importante efeito sobre o band gap dos calcogenetos $\mathrm{Cs}_{2} \mathrm{M}^{\mathrm{II}} \mathrm{M}_{3}^{\mathrm{IV}} \mathrm{Q}_{8}$.

O principal efeito na variação do band gap com mudança na composição é observado com as mudanças no ânion Q. Para essas mudanças, o band gap diminui com o aumento do número atômico de Q. Essa tendência, que é observada em outros compostos calcogenetos, ${ }^{56-57}$ se deve ao aumento da energia dos estados $p$ de Q; como esses estados compõem o topo da banda de valência, esse aumento de energia desloca o MBV para energias maiores, o que reduz o band gap. Os compostos com Sn têm band gaps menores que os compostos com Ge, exceto nos compostos com Te, nos quais as diferenças são menores que 0,15 eV. A energia dos estados $s$ do átomo de Sn é maior que de Ge, o que poderia causar maiores energias do MBC e consequentemente maiores band gaps. Entretanto, devido a efeitos nos níveis de energia eletrônicos, variações de volume da célula causam mudanças no band gap, e resultados mostram que o aumento de volume diminui o band gap em vários semicondutores ${ }^{58}$ e portanto esse efeito contribui para gerar a tendência observada. Por 
Estruturas PBE

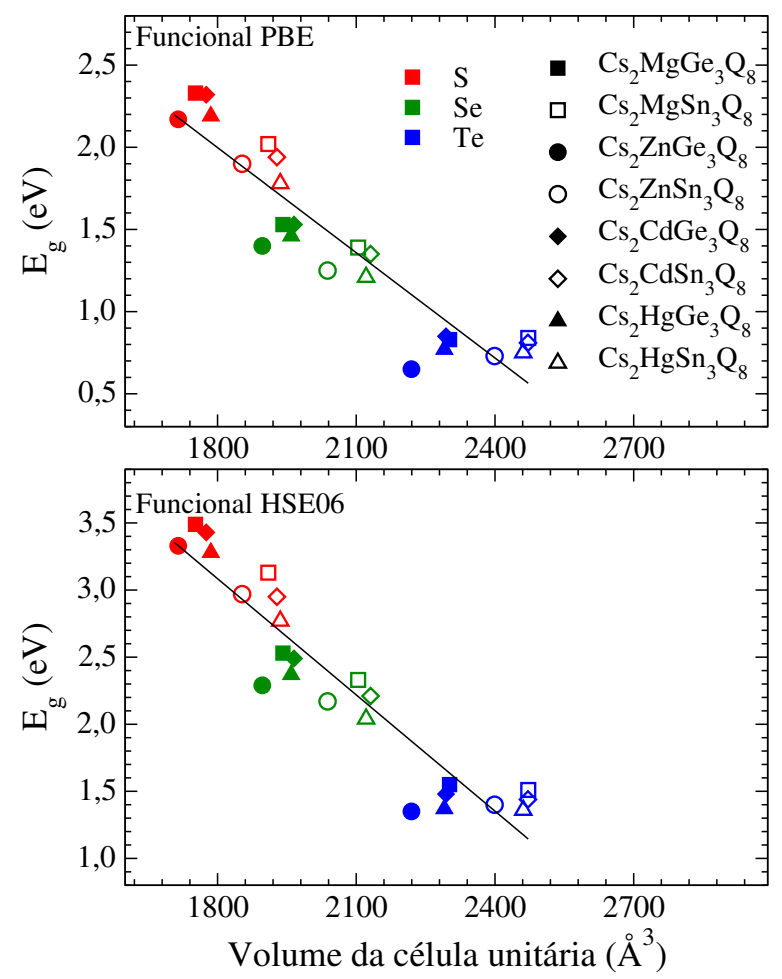

Estruturas PBE+TS+SCS
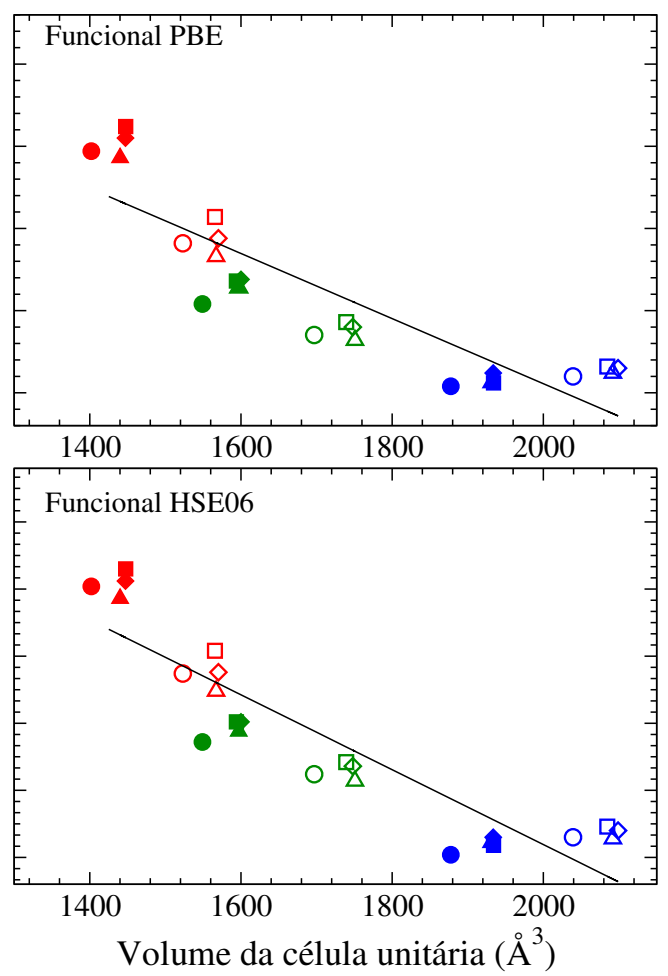

Figura 14 - Valores de band gap no ponto $\Gamma$ em função do volume da célula unitária para os 24 calcogenetos $\mathrm{Cs}_{2} \mathrm{M}^{\mathrm{II}} \mathrm{M}_{3}^{\mathrm{IV}} \mathrm{Q}_{8}\left(\mathrm{M}^{\mathrm{II}}=\mathrm{Mg}, \mathrm{Zn}, \mathrm{Cd}, \mathrm{Hg} ; \mathrm{M}^{\mathrm{IV}}=\mathrm{Ge}, \mathrm{Sn} ; \mathrm{Q}=\mathrm{S}, \mathrm{Se}\right.$, $\mathrm{Te}$ ). Os resultados mostrados na primeira coluna correspondem às estruturas de equilíbrio de $\mathrm{PBE}$, enquanto a segunda coluna contém os resultados das estruturas de $\mathrm{PBE}+\mathrm{TS}+\mathrm{SCS}$. Os resultados da linha superior foram obtidos com o funcional PBE, enquanto os da segunda linha foram obtidos com HSE06.

Fonte: Adaptada de BESSE; SABINO; DA SILVA. ${ }^{45}$

fim, observa-se que modificar o cátion $\mathrm{M}^{\mathrm{II}}$ não afeta significativamente o band gap quando $\mathrm{Q}$ e $\mathrm{M}^{\mathrm{IV}}$ são mantidos fixos, porque os átomos de $\mathrm{M}^{\mathrm{II}}$ têm uma contribuição muito pequena para estados próximos do MBV e MBC, e além disso modificações em $\mathrm{M}^{\mathrm{II}}$ têm efeitos pequenos no volume da célula cristalina.

\subsection{Propriedades ópticas}

Para concluir a discussão das características dos calcogenetos $\mathrm{Cs}_{2} \mathrm{M}^{\mathrm{II}} \mathrm{M}_{3}^{\mathrm{IV}} \mathrm{Q}_{8}$, essa seção concentra-se nas propriedades ópticas desses materiais. Nas interações dos materiais com a radiação eletromagnética, as contribuições mais importantes para regiões de frequência do ultravioleta e da luz visível se originam de modificações na configuração eletrônica, com deslocamentos das nuvens de carga dos elétrons. ${ }^{40}$ Portanto, os processos ópticos em semicondutores ocorrem através de transições eletrônicas entre as bandas de energia e a análise das propriedades ópticas deve envolver a descrição dessas transições. A probabilidade das transições ocorrerem é descrita pelos elementos da matriz de transição, 

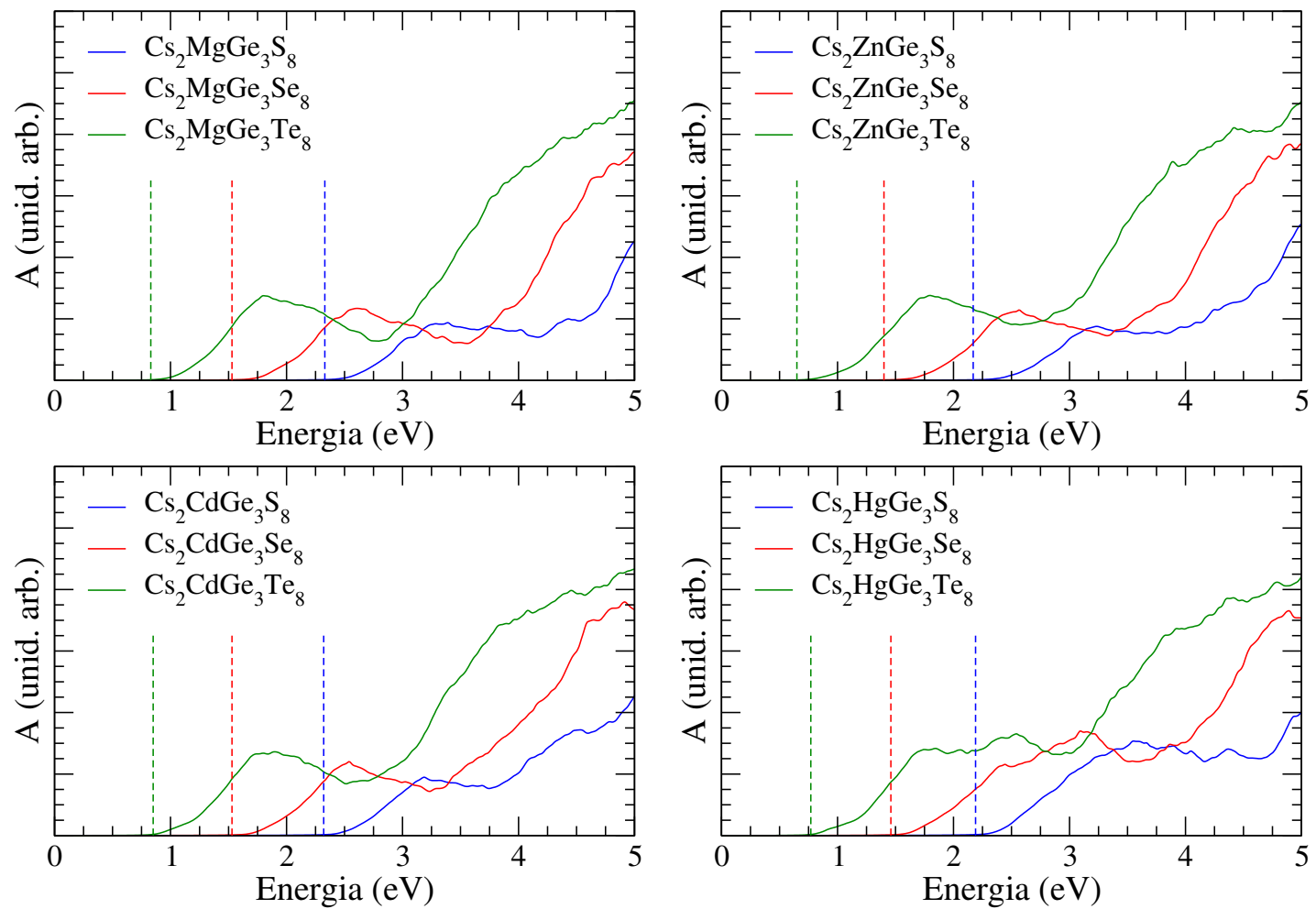

Figura 15 - Coeficientes de absorção óptica dos 24 calcogenetos $\mathrm{Cs}_{2} \mathrm{M}^{\mathrm{II}} \mathrm{M}_{3}^{\mathrm{IV}} \mathrm{Q}_{8}\left(\mathrm{M}^{\mathrm{II}}=\mathrm{Mg}, \mathrm{Zn}\right.$, $\mathrm{Cd}, \mathrm{Hg} ; \mathrm{M}^{\mathrm{IV}}=\mathrm{Ge}, \mathrm{Sn} ; \mathrm{Q}=\mathrm{S}, \mathrm{Se}, \mathrm{Te}$ ) obtidos com PBE. As curvas mostradas são as médias das 3 direções de polarização $(x, y, z)$. As linhas verticais tracejadas indicam o band gap eletrônico no ponto $\Gamma$ dos compostos.

Fonte: Adaptada de BESSE; SABINO; DA SILVA. ${ }^{45}$

$\left\langle\psi_{i}\left|\hat{\mathbf{p}} \cdot \hat{\mathbf{e}}_{\alpha}\right| \psi_{f}\right\rangle$, entre os estados $\psi_{i}$ e $\psi_{f}$, onde $\hat{p}$ é o operador momento e $\alpha$ é uma direção de polarização. ${ }^{59-60}$ Isso explicita a forma como as transições dependem das propriedades dos estados eletrônicos em cada sistema. Além disso, pode haver situações em que determinadas transições tenham elemento de matriz correspondente nulo, ou seja, tais transições não devem ocorrer, e são ditas proibidas. Por isso, distingue-se o band gap eletrônico, discutido anteriormente, do band gap óptico, que é a menor diferença de energia entre estados com transição permitida. Materiais nos quais existe uma diferença entre os band gaps óptico e fundamental, como é o caso dos óxidos transparentes condutores, são importantes para diversas aplicações tecnológicas. ${ }^{61-62}$

$\mathrm{O}$ band gap óptico dos calcogenetos $\mathrm{Cs}_{2} \mathrm{M}^{\mathrm{II}} \mathrm{M}_{3}^{\mathrm{IV}} \mathrm{Q}_{8}$ foi determinado pela análise dos elementos de matriz de transição no ponto $\Gamma$, à semelhança dos band gaps eletrônicos, que foram calculados como diretos no ponto $\Gamma$. Essa análise foi feita em 6 compostos $\left(\mathrm{Cs}_{2} \mathrm{CdGe}_{3} \mathrm{~S}_{8}, \mathrm{Cs}_{2} \mathrm{CdGe}_{3} \mathrm{Se}_{8}, \mathrm{Cs}_{2} \mathrm{CdGe}_{3} \mathrm{Te}_{8}, \mathrm{Cs}_{2} \mathrm{CdSn}_{3} \mathrm{~S}_{8}, \mathrm{Cs}_{2} \mathrm{MgGe}_{3} \mathrm{~S}_{8}\right.$ e $\left.\mathrm{Cs}_{2} \mathrm{MgSn}_{3} \mathrm{Se}_{8}\right)$ e mostrou que as diferenças entre os band gaps óptico e fundamental são menores que $0,1 \mathrm{eV}$, e portanto não são importantes quando comparadas, por exemplo, à disparidade de $0,8 \mathrm{eV}$ observada em $\mathrm{In}_{2} \mathrm{O}_{3} \cdot{ }^{63}$ Esse resultado, que pode ser estendido para os demais compos- 
tos $\mathrm{Cs}_{2} \mathrm{M}^{\mathrm{II}} \mathrm{M}_{3}^{\mathrm{IV}} \mathrm{Q}_{8}$, pode ser interpretado com base em condições de simetria aplicadas recentemente a óxidos transparentes condutores. ${ }^{62}$ Pela análise da tabela de caracteres do grupo pontual $D_{2}$, que descreve as simetrias da estrutura, somente são proibidas transições entre estados com mesma representação. Assim, devido ao grande número de bandas com energias similares que existem próximas de MBV e MBC, é grande o número de transições possíveis e a possibilidade de haver uma grande diferença de energia entre o band gap fundamental e a primeira transição permitida é pequena.

Com os elementos da matriz de transição, é determinada a parte imaginária do tensor dielétrico dependente fa frequência $(\omega)$, tomando todas as transições de estados ocupados para estados vazios, e somando sobre os pontos $\mathbf{k},{ }^{59-60}$

$$
\operatorname{Im}\left(\epsilon_{\alpha \beta}(\omega)\right)=\left(\frac{2 \pi}{\omega}\right)^{2} \sum_{i, j} \sum_{\mathbf{k}}\left\langle\psi_{i}\left|\hat{\mathbf{p}} \cdot \hat{\mathbf{e}}_{\alpha}\right| \psi_{f}\right\rangle\left\langle\psi_{f}\left|\hat{\mathbf{p}} \cdot \hat{\mathbf{e}}_{\beta}\right| \psi_{i}\right\rangle \delta\left(\varepsilon_{f}(\mathbf{k})-\varepsilon_{i}(\mathbf{k})-\omega\right)
$$

enquanto a parte real é obtida pela transformação de Kramers-Kronig. ${ }^{64}$ Da função dielétrica, foi obtido o coeficiente de absorção óptica, ${ }^{59-60}$

$$
A_{\alpha \alpha}(\omega)=\frac{\omega}{c} \sqrt{2\left[\left|\epsilon_{\alpha \alpha}(\omega)\right|-\operatorname{Re}\left(\epsilon_{\alpha \alpha}(\omega)\right)\right]},
$$

que define a taxa de decaimento da intensidade da radiação ao penetrar no material, em função da energia. As curvas do coeficiente de absorção óptica para os 24 compostos são mostradas na figura 15, onde também são indicados os valores do band gap eletrônico no ponto $\Gamma$. Os coeficientes de absorção dependem da direção de polarização, mas os resultados dos $\mathrm{Cs}_{2} \mathrm{M}^{\mathrm{II}} \mathrm{M}_{3}^{\mathrm{IV}} \mathrm{Q}_{8}$ são muito similares nas três direções e portanto são mostrados valores médios. Os gráficos da figura 15 retomam o resultado da similaridade entre os band gaps óptico e eletrônico, pois em todos os casos o começo da curva de absorção, que indica o band gap óptico, está em um valor de energia muito próximo do band gap eletrônico. Em todas as curvas, existem regiões de energia nas quais a absorção deixa de aumentar e até diminui. Esse comportamento é consequência dos intervalos de energia que existem entre bandas sucessivas no mesmo ponto $\mathbf{k}$, na região das bandas não ocupadas, conforme pode ser visto nas energias mais altas das estruturas de bandas nas figuras 9 e 10 .

\subsection{Conclusão}

Foram obtidas e discutidas as propriedades estruturais, energéticas, eletrônicas e ópticas dos 24 calcogenetos $\mathrm{Cs}_{2} \mathrm{M}^{\mathrm{II}} \mathrm{M}_{3}^{\mathrm{IV}} \mathrm{Q}_{8}\left(\mathrm{M}^{\mathrm{II}}=\mathrm{Mg}, \mathrm{Zn}, \mathrm{Cd}, \mathrm{Hg} ; \mathrm{M}^{\mathrm{IV}}=\mathrm{Ge}\right.$, Sn; $\mathrm{Q}=\mathrm{S}$, Se, Te) na estrutura cristalina ortorrômbica. Os parâmetros de rede obtidos variam com a composição de acordo com os raios atômicos e estão em bom acordo com valores experimentais disponíveis, com exceção do parâmetro relacionado à distâncias entre camadas. A variação da energia de coesão entre um composto e outro foi correlacionada com o caráter iônico dos sistemas, e os resultados de energia de ligação entre camadas ressaltaram a importância das interações de van der Waals. O cálculo de propriedades 
eletrônicas permitiu observar nos 24 compostos a diversidade de valores de band gap, cuja diminuição com o aumento do raio atômico de Q foi explicada com base no aumento da energia dos estados $p$ de Q. Através da análise dos elementos de matriz de transição óptica e coeficientes de absorção, concluiu-se que não existe diferença significativa entre o band gap fundamental e o óptico nos materiais $\mathrm{Cs}_{2} \mathrm{M}^{\mathrm{II}} \mathrm{M}_{3}^{\mathrm{IV}} \mathrm{Q}_{8}$. 


\section{EFEITO DOS ÁTOMOS DE METAIS ALCALINOS E CALCOGÊNIOS NA ES- TABILIDADE RELATIVA DAS ESTRUTURAS CRISTALINAS DOS CALCO- GENETOS QUATERNÁRIOS $\mathrm{A}_{2} \mathbf{Z n S n}_{3} \mathbf{Q}_{8}$}

\subsection{Introdução}

Conforme discutido no capítulo 2, dois tipos de estruturas cristalinas foram observados nos calcogenetos $\mathrm{A}_{2} \mathrm{ZnSn}_{3} \mathrm{Q}_{8}$. O composto $\mathrm{K}_{2} \mathrm{ZnSn}_{3} \mathrm{~S}_{8}$ possui uma estrutura formada por empilhamento de camadas e outra formada por uma rede tridimensional fechada, sem camadas. ${ }^{19}$ Por outro lado, as duas estruturas cristalinas observadas nos compostos $\mathrm{Cs}_{2} \mathrm{M}^{\mathrm{II}} \mathrm{M}_{3}^{\mathrm{IV}} \mathrm{Q}_{8}$, têm formação de camadas. ${ }^{20}$ Por isso, é importante investigar o papel dos raios atômicos na estabilidade dessas estruturas, a fim de identificar fatores que influenciam na formação de estruturas cristalinas em camadas nos calcogenetos quaternários. Este capítulo concentra-se na discussão da estabilidade relativa de três estruturas cristalinas distintas dos calcogenetos $\mathrm{A}_{2} \mathrm{ZnSn}_{3} \mathrm{Q}_{8}$ contendo diferentes $\mathrm{A}$ e Q. Ele está dividido nas seções: (4.2) Estruturas cristalinas; (4.3) Parâmetros de rede de equilíbrio; (4.4) Estabilidade relativa das estruturas.

\subsection{Estruturas cristalinas}

As estruturas cristalinas cúbica e triclínica, observadas em $\mathrm{K}_{2} \mathrm{ZnSn}_{3} \mathrm{~S}_{8}$, estão representadas na figura 16, junto com a estrutura ortorrômbica dos compostos $\mathrm{Cs}_{2} \mathrm{M}^{\mathrm{II}} \mathrm{M}_{3}^{\mathrm{IV}} \mathrm{Q}_{8}$. Para diferenciar os tipos de estruturas, 2D e 3D são usados para denotar estruturas com e sem a formação de camadas, respectivamente. Portanto, nesse capítulo as estruturas são denominadas 2D-ortorrômbica, 2D-triclínica e 3D-cúbica. A estrutura 2D-triclínica, que tem grupo espacial $P \overline{1}$ e 2 f.u. por célula unitária, é muito similar à estrutura 2Dortorrômbica dos compostos $\mathrm{Cs}_{2} \mathrm{M}^{\mathrm{II}} \mathrm{M}_{3}^{\mathrm{IV}} \mathrm{Q}_{8}$ e possui empilhamento de camadas na direção [011], em que cada camada tem configuração geométrica praticamente idêntica às camadas

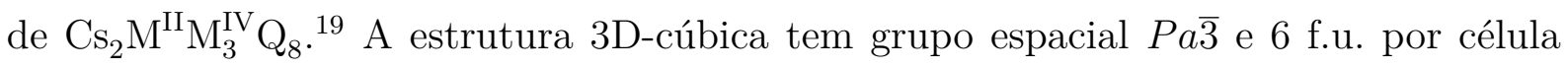
unitária. Ela é constituída por uma rede tridimensional aniônica formada por tetraedros $\mathrm{MQ}_{4}(\mathrm{M}=\mathrm{Zn}$, Sn, com ocupação de $25 \%$ e $75 \%$, respectivamente) interconectados pelos vértices, enquanto os cátions $\mathrm{K}^{+}$ocupam vazios entre os tetraedros. ${ }^{19}$

Devido à ocupação desordenada dos átomos de Zn e Sn nos mesmos sítios dos tetraedros $\mathrm{MQ}_{4}$ na estrutura 3D-cúbica, para modelar essa estrutura é necessário determinar como esses sítios são ocupados. Para as configurações nas quais os átomos de Zn estão próximos, espera-se uma menor estabilidade comparada com a de configurações em que esses átomos estão mais distantes entre si na célula unitária, devido à diferença de tamanho entre os átomos de Zn e Sn. Isso é confirmado pelos testes realizados, que mostraram que uma estrutura $3 \mathrm{D}$-cúbica de $\mathrm{K}_{2} \mathrm{ZnSn}_{3} \mathrm{~S}_{8}$ com átomos de $\mathrm{Zn}$ próximos tem energia 


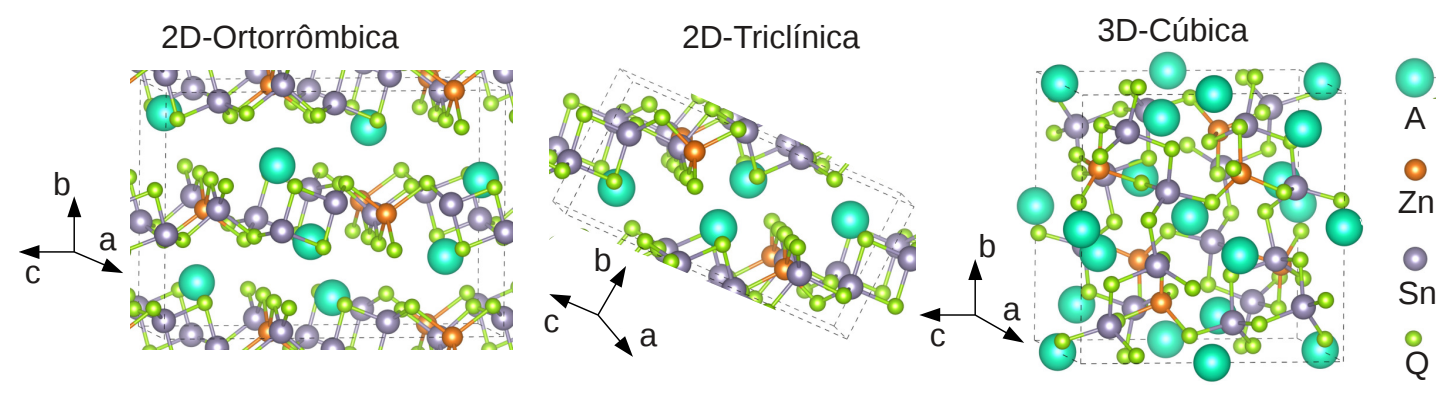

Figura 16 - Representação das estruturas cristalinas 2D-ortorrômbica, 2D-triclínica e 3D-cúbica dos compostos $\mathrm{A}_{2} \mathrm{M}^{\mathrm{II}} \mathrm{M}_{3}^{\mathrm{IV}} \mathrm{Q}_{8}$

Fonte: Elaborada pelo autor.

0,864 eV/f.u. maior que uma estrutura na qual tetraedros $\mathrm{ZnS}_{4}$ não compartilham vértices. Portanto, essa segunda configuração foi adotada para os cálculos da estrutura 3D-cúbica. Além disso, para a minimização da energia, essa estrutura foi otimizada sem os vínculos de uma rede cúbica, o que levou a pequenos desvios de ângulos em relação a $90^{\circ}$ e pequenas diferenças entre os parâmetros de uma mesma estrutura.

Para estudar o efeito dos raios atômicos na estabilidade relativa, as estruturas 2D-ortorrômbica, 2D-triclínica e 3D-cúbica foram otimizadas nos compostos $\mathrm{A}_{2} \mathrm{ZnSn}_{3} \mathrm{Q}_{8}$, com $\mathrm{A}=\mathrm{Li}, \mathrm{K}, \mathrm{Cs} ; \mathrm{Q}=\mathrm{S}$, Se, Te. Os metais $\mathrm{M}^{\mathrm{II}}$ e $\mathrm{M}^{\mathrm{IV}}$ foram mantidos fixos em $\mathrm{Zn}$ e Sn pois, conforme observado entre os compostos $\mathrm{Cs}_{2} \mathrm{M}^{\mathrm{II}} \mathrm{M}_{3}^{\mathrm{IV}} \mathrm{Q}_{8},{ }^{20}$ não se espera que a mudança desses metais afete o tipo de estrutura cristalina adotada. As estruturas foram otimizadas por minimização do tensor de stress e forças atômicas, utilizando o funcional PBE e o método D3 para correções de van der Waals.

\subsection{Parâmetros de rede de equilíbrio}

Na tabela 5, são mostrados os parâmetros de rede de equilíbrio dos 9 compostos nas 3 estruturas. Os maiores desvios observados das estruturas 3D-cúbicas, em relação à rede cúbica, são diferenças de ângulo de $1,2^{\circ}$ em relação a $90^{\circ}$ nos compostos $\operatorname{Li}_{2} Z_{n S n} \mathrm{Se}_{8}$ e $\mathrm{Li}_{2} \mathrm{ZnSn}_{3} \mathrm{Te}_{8}$, além de uma diferença de 0,54 $\AA$ entre os parâmetros de rede de $\mathrm{Li}_{2} \mathrm{ZnSn}_{3} \mathrm{Te}_{8}$. O volume da célula unitária em todos os casos, conforme esperado, aumenta com o aumento de raios atômicos, como já verificado na estrutura ortorrômbica dos compostos $\mathrm{Cs}_{2} \mathrm{M}^{\mathrm{II}} \mathrm{M}_{3}^{\mathrm{IV}} \mathrm{Q}_{8}$. Em comparação com os resultados experimentais, os resultados de parâmetros de rede apresentam boa concordância, conforme se observa na tabela 6. Para $\mathrm{K}_{2} \mathrm{ZnSn}_{3} \mathrm{~S}_{8}$, as diferenças relativas são menores que $1,0 \%$ e 1,8\% para as estruturas 2Dtriclínica e 3D-cúbica, respectivamente, enquanto que para a estrutura 2D-ortorrômbica de $\mathrm{Cs}_{2} \mathrm{ZnSn}_{3} \mathrm{Se}_{8}$ as diferenças são menores que 1,0\%. Essas diferenças indicam que esses resultados são substancialmente melhores que os obtidos para os compostos $\mathrm{Cs}_{2} \mathrm{M}^{\mathrm{II}} \mathrm{M}_{3}^{\mathrm{IV}} \mathrm{Q}_{8}$ com PBE+TS+SCS, apresentados no capítulo anterior. 
Tabela 5 - Parâmetros de rede de equilíbrio e energias totais relativas dos materiais $\mathrm{A}_{2} \mathrm{ZnSn}_{3} \mathrm{Q}_{8}$ $(\mathrm{A}=\mathrm{Li}, \mathrm{K}, \mathrm{Cs} ; \mathrm{Q}=\mathrm{S}, \mathrm{Se}, \mathrm{Te})$ nas estruturas 2D-triclínica, 3D-cúbica e 2Dortorrômbica

\begin{tabular}{|c|c|c|c|c|c|c|c|c|}
\hline Cor & Estrutura & $\begin{array}{c}a_{0} \\
(\AA)\end{array}$ & $\begin{array}{c}b_{0} \\
(\AA)\end{array}$ & $\begin{array}{c}c_{0} \\
(\AA)\end{array}$ & $\begin{array}{c}\alpha \\
\left(^{\circ}\right)\end{array}$ & $\begin{array}{l}\beta \\
\left({ }^{\circ}\right)\end{array}$ & $\begin{array}{c}\gamma \\
\left(^{\circ}\right)\end{array}$ & $\begin{array}{c}\Delta E_{t o t} \\
\text { (meV/f.u.) }\end{array}$ \\
\hline \multirow{3}{*}{$\mathrm{Li}_{2} \mathrm{ZnSn}_{3} \mathrm{~S}_{8}$} & 2D-tric. & 7,38 & 7,04 & 15,76 & 96,1 & 84,6 & 120,3 & 0 \\
\hline & 3D-cub. & 12,67 & 12,33 & 12,73 & 89,1 & 89,8 & 91,1 & 598 \\
\hline & 2D-ort. & 7,17 & 11,04 & 17,21 & 90,0 & 90,0 & 90,0 & 328 \\
\hline \multirow[t]{3}{*}{$\mathrm{Li}_{2} \mathrm{ZnSn}_{3} \mathrm{Se}_{8}$} & 2D-tric. & 7,63 & 7,39 & 16,25 & 96,0 & 84,9 & 118,8 & 0 \\
\hline & 3D-cub. & 13,12 & 12,81 & 13,28 & 89,7 & 90,1 & 91,2 & 397 \\
\hline & 2D-ortho. & 7,44 & 11,51 & 17,93 & 90,0 & 90,0 & 90,0 & 182 \\
\hline \multirow[t]{3}{*}{$\mathrm{Li}_{2} \mathrm{ZnSn}_{3} \mathrm{Te}_{8}$} & 2D-tric. & 7,98 & 7,90 & 17,09 & 99,1 & 81,9 & 115,2 & 0 \\
\hline & 3D-cub. & 13,92 & 13,72 & 14,26 & 89,7 & 90,4 & 91,2 & 383 \\
\hline & 2D-ort. & 7,93 & 12,30 & 19,16 & 90,0 & 90,0 & 90,0 & 185 \\
\hline \multirow[t]{3}{*}{$\mathrm{K}_{2} \mathrm{ZnSn}_{3} \mathrm{~S}_{8}$} & 2D-tric. & 7,33 & 7,93 & 15,35 & 95,2 & 91,3 & 114,6 & 0 \\
\hline & 3D-cub. & 13,36 & 13,19 & 13,22 & 90,1 & 90,1 & 90,0 & -77 \\
\hline & 2D-ort. & 7,35 & 12,18 & 17,73 & 90,0 & 90,0 & 90,0 & -1 \\
\hline \multirow[t]{3}{*}{$\mathrm{K}_{2} \mathrm{ZnSn}_{3} \mathrm{Se}_{8}$} & 2D-tric. & 7,59 & 8,26 & 15,98 & 94,8 & 92,6 & 116,0 & 0 \\
\hline & 3D-cub. & 13,90 & 13,70 & 13,76 & 90,0 & 90,2 & 89,9 & 45 \\
\hline & 2D-ort. & 7,62 & 12,59 & 18,44 & 90,0 & 90,0 & 90,0 & -35 \\
\hline \multirow[t]{3}{*}{$\mathrm{K}_{2} \mathrm{ZnSn}_{3} \mathrm{Te}_{8}$} & 2D-tric. & 8,06 & 8,96 & 16,90 & 92,4 & 94,7 & 118,8 & 0 \\
\hline & 3D-cub. & 14,78 & 14,60 & 14,69 & 89,4 & 90,4 & 89,9 & 165 \\
\hline & 2D-ort. & 8,12 & 13,34 & 19,62 & 90,0 & 90,0 & 90,0 & -57 \\
\hline \multirow[t]{3}{*}{$\mathrm{Cs}_{2} \mathrm{ZnSn}_{3} \mathrm{~S}_{8}$} & 2D-tric. & 7,59 & 7,97 & 15,61 & 93,1 & 97,1 & 110,9 & 0 \\
\hline & 3D-cub. & 13,77 & 13,65 & 13,65 & 90,1 & 90,0 & 90,2 & -26 \\
\hline & 2D-ort. & 7,61 & 12,54 & 17,94 & 90,0 & 90,0 & 90,0 & -34 \\
\hline \multirow{3}{*}{$\mathrm{Cs}_{2} \mathrm{ZnSn}_{3} \mathrm{Se}_{8}$} & 2D-tric. & 7,89 & 8,20 & 16,45 & 91,7 & 100,8 & 114,0 & 0 \\
\hline & 3D-cub. & 14,33 & 14,15 & 14,23 & 89,9 & 90,2 & 90,1 & 129 \\
\hline & 2D-ort. & 7,88 & 12,79 & 18,62 & 90,0 & 90,0 & 90,0 & -69 \\
\hline \multirow[t]{3}{*}{$\mathrm{Cs}_{2} \mathrm{ZnSn}_{3} \mathrm{Te}_{8}$} & 2D-triC. & 8,36 & 8,99 & 17,45 & 90,2 & 100,3 & 118,6 & 0 \\
\hline & 3D-cub. & 15,25 & 15,02 & 15,15 & 89,5 & 90,2 & 90,0 & 294 \\
\hline & 2D-ort. & 8,39 & 13,56 & 19,62 & 90,0 & 90,0 & 90,0 & -104 \\
\hline
\end{tabular}

Fonte: BESSE; DA SILVA. ${ }^{65}$

\subsection{Estabilidade relativa das estruturas}

Para avaliar a estabilidade relativa das estruturas, o critério utilizado foi a comparação das energias totais por fórmula unitária das 3 estruturas de um mesmo composto, sendo que a estabilidade é favorecida por menores energias. Na tabela 5 são apresentados os resultados de energias totais relativas $\left(\Delta E_{t o t}\right)$ para todos os sistemas, com a estrutura 2D-triclínica de cada compsoto adotada como referência. Os resultados indicam que para $\mathrm{K}_{2} \mathrm{ZnSn}_{3} \mathrm{~S}_{8}$ a estrutura de menor energia é a 3D-cúbica, enquanto a estrutura 2D-ortorrômbica é a de menor energia para $\mathrm{Cs}_{2} \mathrm{ZnSn}_{3} \mathrm{Se}_{8}$. Portanto, os cálculos fornecem as estruturas mais estáveis em acordo com as observações experimentais. ${ }^{19-20}$ 
Tabela 6 - Comparação entre os resultados dos parâmetros de rede obtidos nesse trabalho e os dados experimentais de $\mathrm{K}_{2} \mathrm{ZnSn}_{3} \mathrm{~S}_{8}$ nas estruturas 2D-triclínica e 3D-cúbica, e estrutura 2D-ortorrômbica de $\mathrm{Cs}_{2} \mathrm{ZnSn}_{3} \mathrm{~S}_{8}$.

\begin{tabular}{|c|c|c|c|c|c|c|}
\hline \multirow[b]{2}{*}{ Parâmetro } & \multicolumn{2}{|c|}{$\begin{array}{l}\text { 2D-triclínica } \\
\mathrm{K}_{2} \mathrm{ZnSn}_{3} \mathrm{~S}_{8}\end{array}$} & \multicolumn{2}{|c|}{$\begin{array}{l}\text { 3D-cúbica } \\
\mathrm{K}_{2} \mathrm{ZnSn}_{3} \mathrm{~S}_{8}\end{array}$} & \multicolumn{2}{|c|}{$\begin{array}{l}\text { 2D-ortorrômbica } \\
\mathrm{Cs}_{2} \mathrm{ZnSn}_{3} \mathrm{Se}_{8}\end{array}$} \\
\hline & Calculado & Exp. $^{19}$ & Calculado & Exp. $^{19}$ & Calculado & Exp. $^{20}$ \\
\hline$a_{0}(\AA)$ & 7,33 & 7,37 & 13,36 & 13,13 & 7,88 & 7,81 \\
\hline$b_{0}(\AA)$ & 7,93 & 8,00 & 13,19 & 13,13 & 12,79 & 12,65 \\
\hline$c_{0}(\AA)$ & 15,35 & 15,32 & 13,22 & 13,13 & 18,62 & 18,37 \\
\hline$\alpha\left(^{\circ}\right)$ & 95,2 & 95,0 & 90,1 & 90,0 & 90,0 & 90,0 \\
\hline$\beta\left(^{\circ}\right)$ & 91,3 & 91,6 & 90,1 & 90,0 & 90,0 & 90,0 \\
\hline$\gamma\left(^{\circ}\right)$ & 114,6 & 115,8 & 90,0 & 90,0 & 90,0 & 90,0 \\
\hline
\end{tabular}

Fonte: FARD; KANATZIDIS; ${ }^{19}$ BESSE; DA SILVA. ${ }^{65}$

A estabilidade relativa das estruturas é influenciada pelas mudanças na composição, nas quais o raio atômico das espécies A e Q tem um importante papel. Esse comportamento é demonstrado na figura 17, que mostra os valores de energia relativa das estruturas em função da média dos raios atômicos das espécies A e Q em cada composto. Os raios atômicos foram atribuídos de forma distinta em cada um dos gráficos: em (a), foram usados valores de referência para raios iônicos, ${ }^{40}$ e em (b) os raios atômicos foram obtidos a partir dos valores de distâncias médias de ligação, calculadas seguindo o método descrito no Capítulo 3, e usando o modelo de esferas rígidas, $R=d_{m e d} / 2$. A similaridade entre os dois gráficos evidencia o papel dos raios atômicos. Neles, é possível observar a diferença entre os comportamentos da estrutura 2D-ortorrômbica em relação à estrutura 3D-cúbica. A estrutura ortorrômbica exibe um decaimento aproximadamente linear da energia com o aumento da média dos raios atômicos de $\mathrm{A}$ e $\mathrm{Q}$, enquanto os valores correspondentes à estrutura 3D-cúbica são maiores na região de raios atômicos pequenos $(R<1,5 \AA$ ), que corresponde aos compostos com $\mathrm{A}=\mathrm{Li}$, e também na região de raios atômicos grandes ( $R>1,8 \AA$ ), deixando valores mínimos para os compostos com $\mathrm{A}=\mathrm{K}$. Como consequência desses comportamentos, obtêm-se que a estrutura 2D-triclínica é mais estável para raios pequenos, enquanto a 2D-ortorrômbica é a mais estável para raios grandes, e entre essas regiões situa-se o composto $\mathrm{K}_{2} \mathrm{ZnSn}_{3} \mathrm{~S}_{8}$ para o qual a estrutura 3D-cúbica tem menor energia.

As tendências observadas podem ser melhor entendidas ao analisar as características de cada composto e estrutura. Nos compostos com A $=\mathrm{K}$ ou Cs, as estruturas 2D são cada vez mais favorecidas em relação à estrutura $3 \mathrm{D}$ à medida que os raios atômicos de A e Q aumentam, isto é, as diferenças de energia entre a estrutura 3D-cúbica e as estruturas 2D-ortorrômbica e 2D-triclínica ficam cada vez maiores. Por exemplo, enquanto a estrutura $3 \mathrm{D}$-cúbica de $\mathrm{K}_{2} \mathrm{ZnSn}_{3} \mathrm{Se}_{8}$ tem energia $45 \mathrm{meV} /$ f.u. maior que a 2D-triclínica, 

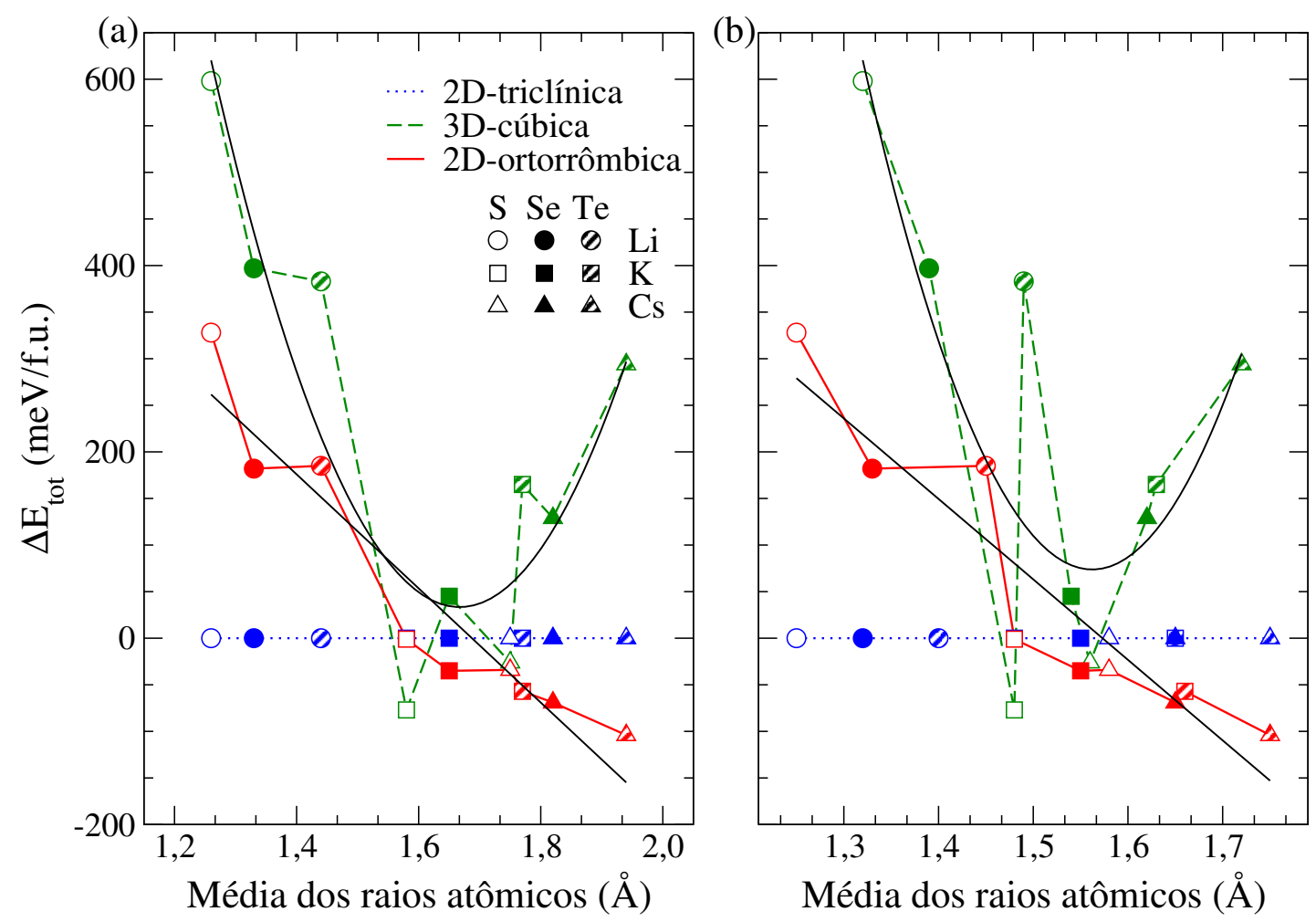

Figura 17 - Energia total relativa das estruturas 2D-triclínica, 3D-cúbica e 2D-ortorrômbica dos compostos $\mathrm{A}_{2} \mathrm{ZnSn}_{3} \mathrm{Q}_{8}(\mathrm{~A}=\mathrm{Li}, \mathrm{K}, \mathrm{Cs} ; \mathrm{Q}=\mathrm{S}$, Se, Te), em função da média dos raios atômicos de A e Q. Em (a), os raios atômicos usados são valores de raios iônicos de referência, ${ }^{40}$ e em (b) os raios atômicos foram obtidos a partir dos valores de distâncias médias de ligação, usando o modelo de esferas rígidas, $R=d_{\text {med }} / 2$.

Fonte: Adaptada de BESSE; DA SILVA. ${ }^{65}$

essa diferença é de $165 \mathrm{meV} /$ f.u. em $\mathrm{K}_{2} \mathrm{ZnSn}_{3} \mathrm{Te}_{8}$ e de $129 \mathrm{meV} /$ f.u. em $\mathrm{Cs}_{2} \mathrm{ZnSn}_{3} \mathrm{Se}_{8}$. Para compreender esse comportamento, é útil retomar as diferenças entre as características das estruturas cristalinas. Nas estruturas 2D, os átomos de A se localizam entre as camadas, enquanto a estrutura 3D é fechada, na qual os átomos de A ocupam os vazios formados pela rede $3 \mathrm{D}$ dos tetraedros $\mathrm{MQ}_{4}$. Portanto, o aumento dos raios atômicos é mais facilmente incorporado, isto é, com menores deslocamentos, nas estruturas 2D em comparação com a $3 \mathrm{D}$, o que leva ao maior favorecimento das estruturas $2 \mathrm{D}$ com o aumento dos raios atômicos.

O favorecimento da estrutura 2D-triclínica em relação à 2D-ortorrômbica, que é mais simétrica, com a diminuição dos raios atômicos repete a tendência já reportada na literatura de estruturas menos simétricas serem favorecidas para átomos menores. Por exemplo, na família de calcogenetos quaternários $\mathrm{AGaSnSe}_{4}, \mathrm{KGaSnSe}_{4}$ e $\mathrm{RbGaSnSe}_{4}$ adotam uma estrutura cristalina com rede monoclínica, enquanto $\mathrm{CsGaSnSe}_{4}$ adota uma estrutura com rede ortorrômbica, ${ }^{66}$ e também nos compostos $\mathrm{Cs}_{2} \mathrm{M}^{\mathrm{II}} \mathrm{M}_{3}^{\mathrm{IV}} \mathrm{Q}_{8}$, em que o composto sintetizado que tem os menores átomos cristaliza em uma estrutura monoclínica. ${ }^{20}$ 
Nos compostos com $\mathrm{A}=\mathrm{Li}$, o comportamento é diferente do observado para $\mathrm{A}$ $=\mathrm{K}$, Cs, porque o raio iônico de Li é muito menor em comparação a K e Cs (valores de 0,68 $\AA$ para Li, 1,33 $\AA$ para K e 1,67 $\AA$ para Cs). ${ }^{40}$ Nesses compostos, a estrutura cúbica tem energias altas e portanto estabilidade reduzida, e as energias diminuem com o aumento do raio atômico dos Q. Devido ao menor tamanho dos átomos de Li, a blindagem que eles geram para as repulsões coulombianas da rede aniônica formada pelos tetraedros $\mathrm{MQ}_{4}$ é insuficiente, o que desfavorece a estabilidade da estrutura, já que a rede aniônica não tem a flexibilidade para ser ajustada com as mudanças necessárias, ou seja, as posições dos átomos não podem ser muito modificadas mantendo $\mathrm{M}$ e $\mathrm{Q}$ fixos. ${ }^{67} \mathrm{Com}$ o aumento do raio iônico de $\mathrm{Q}$ esse efeito é diminuído, em acordo com a diminuição observada das energias.

\subsection{Conclusão}

Foram estudadas as estruturas cristalinas 2D-ortorrômbica, 2D-triclínica e 3Dcúbica dos compostos $\mathrm{A}_{2} \mathrm{ZnSn}_{3} \mathrm{Q}_{8}(\mathrm{~A}=\mathrm{Li}, \mathrm{K}, \mathrm{Cs} ; \mathrm{Q}=\mathrm{S}$, Se, Te). As otimizações das estruturas forneceram resultados de parâmetros de rede em boa concordância com os dados experimentais disponíveis. Através da análise da energia das estruturas foi possível observar que os raios iônicos dos cátions de metais alcalinos e ânions calcogênios exercem uma importante influência sobre a estabilidade relativa de estruturas 2D e 3D nos calcogenetos quaternários $\mathrm{A}_{2} \mathrm{ZnSn}_{3} \mathrm{Q}_{8}$. A estrutura 3D-cúbica tem energia mais baixa apenas na região de raio atômico intermediário, correspondente ao composto $\mathrm{K}_{2} \mathrm{ZnSn}_{3} \mathrm{~S}_{8}$, e o favorecimento das estruturas em camadas nos demais casos foi discutido com base na tendência de diminuição de tensões e repulsões coulombianas nas estruturas. 


\section{CONCLUSÃO}

Nesse trabalho, foram realizados cálculos de DFT com o funcional semi-local GGA-PBE e o funcional híbrido HSE06, incluindo as correções de vdW TS+SCS e D3, para estudar a família de calcogenetos quaternários $\mathrm{A}_{2} \mathrm{M}^{\mathrm{II}} \mathrm{M}_{3}^{\mathrm{IV}} \mathrm{Q}_{8}\left(\mathrm{~A}=\mathrm{Li}, \mathrm{K}, \mathrm{Cs} ; \mathrm{M}^{\mathrm{II}}\right.$ $=\mathrm{Mg}, \mathrm{Zn}, \mathrm{Cd}, \mathrm{Hg} ; \mathrm{M}^{\mathrm{IV}}=\mathrm{Ge} \mathrm{Sn} ; \mathrm{Q}=\mathrm{S}$, Se, Te). Foram determinadas propriedades estruturais, energéticas, eletrônicas e ópticas dos 24 compostos $\mathrm{Cs}_{2} \mathrm{M}^{\mathrm{II}} \mathrm{M}_{3}^{\mathrm{IV}} \mathrm{Q}_{8}$ na estrutura 2D-ortorrômbica, e a estabilidade relativa das estruturas 2D-ortorrômbica, 2D-triclínica e 3D-cúbica foi analisada em 9 compostos $\mathrm{A}_{2} \mathrm{ZnSn}_{3} \mathrm{Q}_{8}$.

Entre os compostos $\mathrm{Cs}_{2} \mathrm{M}^{\mathrm{II}} \mathrm{M}_{3}^{\mathrm{IV}} \mathrm{Q}_{8}$ observou-se que os parâmetros de rede da célula unitária têm a correlação esperada com os raios atômicos, isto é, o volume da célula aumenta conforme os raios atômicos aumentam. Os valores calculados para os parâmetros de rede $a_{0}$ e $c_{0}$ estão em bom acordo com os dados experimentais, porém, para o parâmetro $b_{0}$, referente à direção de empilhamento das camadas, cálculos PBE superestimaram os valores entre $6,0 \%$ e 8,0\%, enquanto PBE+TS+SCS subestimou os parâmetros entre $4,0 \%$ e 8,0\%. A variação observada da energia de coesão desses materiais com o número atômico de uma das espécies químicas, junto com a análise das cargas efetivas de Bader, permitiu concluir o importante papel das interações de Coulomb na estabilidade desses compostos. A correção de vdW tem uma importante contribuição na energia de ligação entre as camadas da estrutura cristalina e os valores obtidos de energia de ligação entre camadas por unidade de área estão próximos aos valores reportados na literatura para uma grande quantidade de materiais. Conforme esperado, os band gaps calculados com PBE são consideravelmente menores que os obtidos experimentalmente disponíveis na literatura, enquanto os valores obtidos com o funcional híbrido HSE06 estão em boa concordância com os dados experimentais. Além disso, os resultados permitiram observar, entre os band gaps dos 24 compostos, uma grande variedade de valores, e uma correlação aproximadamente linear entre band gap e volume da célula unitária. O calcogênio Q tem o maior efeito em variar o band gap, em que o aumento do número atômico de $\mathrm{Q}$ leva à maiores energias dos estados $p$ de Q, que compõem o topo da banda de valência e, portanto, o band gap é diminuído. Também foram calculados os coeficientes de absorção óptica para os materiais e, através de análise dos elementos de matriz de transições ópticas, concluiu-se que não existe diferença significativa entre os band gaps óptico e eletrônico nesses compostos.

Para os 9 compostos $\mathrm{A}_{2} \mathrm{ZnSn}_{3} \mathrm{Q}_{8}$ nas estruturas 2D-ortorrômbica, 2D-triclínica e 3D-cúbica, as diferenças relativas entre os parâmetros de rede calculados e os dados experimentais da literatura são todas menores que 1,8\%. Isso mostra que para esses materiais a correção de vdW através do método D3 fornece uma melhor descrição comparada a TS+SCS. As estruturas 3D-cúbica e 2D-ortorrômbica têm as menores energias para 
$\mathrm{K}_{2} \mathrm{ZnSn}_{3} \mathrm{~S}_{8}$ e $\mathrm{Cs}_{2} \mathrm{ZnSn}_{3} \mathrm{Se}_{8}$, respectivamente, em acordo com as observações experimentais de estruturas mais estáveis disponíveis na literatura. As energias totais relativas calculadas mostraram que a estabilidade relativa das estruturas é afetada pela composição química, em particular, pelos raios atômicos dos metais alcalinos e calcogênios. Dentre as estruturas estudadas, as estruturas em camadas são favorecidas nas regiões de raios atômicos grandes ( $>1,8 \AA)$ e pequenos $(<1,5 \AA)$, enquanto a estrutura 3D-cúbica é favorecida na região intermediária. Esse comportamento foi explicado com base nas menores tensões nas estruturas em camadas para átomos grandes em comparação com a estrutura 3D-cúbica, que é mais fechada. Já nos compostos com $\mathrm{A}=\mathrm{Li}$ há aumento da repulsão entre os átomos da rede aniônica na estrutura 3D-cúbica devido à blindagem de carga insuficiente pelos íons de $\mathrm{Li}$, que têm raio muito menor que $\mathrm{K}$ e $\mathrm{Cs}$, desfavorecendo a estabilidade dessa estrutura.

Portanto, esse trabalho contribuiu para melhorar o entendimento atomístico do papel dos elementos químicos nas propriedades dos calcogenetos quaternários $\mathrm{A}_{2} \mathrm{M}^{\mathrm{II}} \mathrm{M}_{3}^{\mathrm{IV}} \mathrm{Q}_{8}$, o que pode auxiliar na obtenção de novos materiais com propriedades específicas de interesse. Durante o desenvolvimento do mestrado, dois artigos foram publicados com os resultados desse projeto:

- BESSE, R.; SABINO, F. P.; DA SILVA, J. L. F. Electronic structure of layered quaternary chalcogenide materials for band-gap engineering: the example of $\mathrm{Cs}_{2} \mathrm{M}^{\mathrm{II}} \mathrm{M}_{3}^{\mathrm{IV}} \mathrm{Q}_{8}$. Physical Review B, v. 93, n; 16, p. 165205, 2016.

- BESSE, R.; DA SILVA, J. L. F. The role of the alkali and chalcogen atoms on the stability of the layered chalcogenide $\mathrm{A}_{2} \mathrm{M}^{\mathrm{II}} \mathrm{M}_{3}^{\mathrm{IV}} \mathrm{Q}_{8}(\mathrm{~A}=$ alkali-metal; $\mathrm{M}=$ metalcations; $\mathrm{Q}=$ chalcogen) compounds: a density functional theory investigation within van der Waals corrections. Journal of Physics: condensed matter, v. 29, n. 3, p. $035402,2017$. 


\section{REFERÊNCIAS}

1 MORALES-ACEVEDO, A. Thin film CdS/CdTe solar cells: research perspectives. Solar Energy, v. 80, n. 6, p. 675-681, 2006.

2 REPINS, I. et al. 19.9\%-efficient $\mathrm{ZnO} / \mathrm{CdS} / \mathrm{CuInGaSe}{ }_{2}$ solar cell with $81.2 \%$ fill factor. Progress in Photovoltaics: research and applications, v. 16, n. 3, p. 235-239, 2008.

3 TODOROV, T. K.; REUTER, K. B.; MITZI, D. B. High-efficiency solar cell with earth-abundant liquid-processed absorber. Advanced Materials, v. 22, n. 20, p. E156-E159, 2010.

4 WUTTIG, M.; YAMADA, N. Phase-change materials for rewriteable data storage. Nature Materials, v. 6, n. 11, p. 824-832, 2007.

5 PARK, J.-W. et al. Optical properties of pseudobinary GeTe, $\mathrm{Ge}_{2} \mathrm{Sb}_{2} \mathrm{Te}_{5}, \mathrm{GeSb}_{2} \mathrm{Te}_{4}$, $\mathrm{GeSb}_{4} \mathrm{Te}_{7}$, and $\mathrm{Sb}_{2} \mathrm{Te}_{3}$ from ellipsometry and density functional theory. Physical Review B, v. 80, n. 11, p. 115209, 2009.

6 MANOS, M. J.; DING, N.; KANATZIDIS, M. G. Layered metal sulfides: exceptionally selective agents for radioactive strontium removal. Proceedings of the National Academy of Science of the United States of America, v. 105, n. 10, p. 3696-3699, 2008.

7 BRANT, J. A. et al. $\mathrm{Li}_{2} \mathrm{CdGeS}_{4}$, a diamond-like semiconductor with strong second-order optical nonlinearity in the infrared and exceptional laser damage threshold. Chemistry of Materials, v. 26, n. 10, p. 3045-3048, 2014.

8 WU, K.; YANG, Z.; PAN, S. $\mathrm{Na}_{2} \mathrm{Hg}_{3} \mathrm{M}_{2} \mathrm{~S}_{8}(\mathrm{M}=\mathrm{Si}$, Ge, and $\mathrm{Sn})$ : new infrared nonlinear optical materials with strong second harmonic generation effects and high laser-damage thresholds. Chemistry of Materials, v. 28, n. 8, p. 2795-2801, 2016.

9 JOHNSEN, S. et al. $\mathrm{Tl}_{2} \mathrm{Hg}_{3} \mathrm{Q}_{4}(\mathrm{Q}=\mathrm{S}$, Se, and Te): high-density, wide-band-gap semiconductors. Chemistry of Materials, v. 23, n. 19, p. 4375-4383, 2011.

10 LI, X. et al. Syntheses, crystal structures and physical properties of three new chalcogenides: $\mathrm{NaGaGe}_{3} \mathrm{Se}_{8}, \mathrm{~K}_{3} \mathrm{Ga}_{3} \mathrm{Ge}_{7} \mathrm{~S}_{2} \mathrm{O}$, and $\mathrm{K}_{3} \mathrm{Ga}_{3} \mathrm{Ge}_{7} \mathrm{Se}_{2} 0$. Dalton Transactions, v. 45, n. 2 , p. 532-538, 2016.

11 FELDMAN, S. D. et al. Effects of $\mathrm{Cu}$ in CdS/CdTe solar cells studied with patterned doping and spatially resolved luminescence. Applied Physics Letters, v. 85, n. 9, p. 1529-1531, 2004.

12 CHHOWALLA, M. et al. The chemistry of two-dimensional layered transition metal dichalcogenide nanosheets. Nature Chemistry, v. 5, n. 4, p. 263-275, 2013.

13 BHIMANAPATI, G. R. et al. Recent advances in two-dimensional materials beyond graphene. ACS Nano, v. 9, n. 12, p. 11509-11539, 2015.

14 NOVOSELOV, K. S. et al. 2D materials and van der Waals heterostructures. Science, v. 353, n. 6298, p. $461,2016$. 
15 GEIM, A. K.; GRIGORIEVA, I. V. Van der Waals heterostructures. Nature, v. 499, n. 7459, p. 419-425, 2013.

$16 \mathrm{LI}, \mathrm{H}$. et al. $\mathrm{CsCdInQ}_{3}(\mathrm{Q}=\mathrm{Se}, \mathrm{Te})$ : new photoconductive compounds as potential materials for hard radiation detection. Chemistry of Materials, v. 25, n. 10, p. 2089-2099, 2013.

17 ANDROULAKIS, J. et al. Dimensional reduction: a design tool for new radiation detection materials. Advanced Materials, v. 23, n. 36, p. 4163-4167, 2011.

18 MEI, D. et al. $\mathrm{LiGaGe}_{2} \mathrm{Se}_{6}$ : a new IR nonlinear optical material with low melting point. Inorganic Chemistry, v. 51, n. 2, p. 1035-1040, 2012.

19 FARD, Z. H.; KANATZIDIS, M. G. Phase-change materials exhibiting tristability: interconverting forms of crystalline $\alpha^{-}, \beta-$, and glassy $\mathrm{K}_{2} \mathrm{ZnSnS}_{8}$. Inorganic Chemistry, v. 51, n. 15, p. 7963-7965, 2012.

20 MORRIS, C. D. et al. $\mathrm{Cs}_{2} \mathrm{M}^{\mathrm{II}} \mathrm{M}_{3}^{\mathrm{IV}} \mathrm{Q}_{8}(\mathrm{Q}=\mathrm{S}$, Se, Te): an extensive family of layered semiconductors with diverse band gaps. Chemistry of Materials, v. 25, n. 16, p. 3344-3356, 2013.

21 BORN, M.; OPPENHEIMER, J. R. Zur quantentheorie der molekeln. Annalen der Physik, v. 389, p. 457-484, 1927.

22 THOMAS, L. H. The calculation of atomic fields. Mathematical Proceedings of the Cambridge Philosophical Society, v. 23, n. 05, p. 542-548, 1927.

23 PARR, R. G.; YANG, W. Density-functional theory of atoms and molecules. New York: Oxford University Press, 1989.

24 HOHENBERG, P.; KOHN, W. Inhomogeneous electron gas. Physical Review, v. 136, n. 3B, p. B864-B871, 1964.

25 KOHN, W.; SHAM, L. J. Self-consistent equations including exchange and correlation effects. Physical Review, v. 140, n. 4A, p. A1133-A1138, 1965.

26 KRESSE, G.; HAFNER, J. Ab Initio molecular dynamics for open-shell transition metals. Physical Review B, v. 48, n. 17, p. 13115-13118, 1993.

27 KRESSE, G.; FURTHMÜLLER, J. Efficient iterative schemes for Ab Initio total-energy calculations using a plane-wave basis set. Physical Review B, v. 54, n. 16, p. 11169-11186, 1996.

28 PERDEW, J. P.; WANG, Y. Accurate and simple analytic representation of the electron-gas correlation energy. Physical Review B, v. 45, n. 23, p. 13244-13249, 1992.

29 PERDEW, J. P.; BURKE, K.; ERNZERHOF, M. Generalized gradient approximation made simple. Physical Review Letters, v. 77, n. 18, p. 3865-3868, 1996.

30 PERDEW, J. P. et al. Atoms, molecules, solids, and surfaces: applications of the generalized gradient approximation for exchange and correlation. Physical Review B, v. 46, n. 11, p. 6671-6687, 1992. 
31 PERDEW, J. P.; LEVY, M. Physical content of the exact Kohn-Sham orbital energies: band gaps and derivative discontinuities. Physical Review Letters, v. 51, n. 20 , p. $1884-1887,1983$.

32 HEYD, J.; SCUSERIA, G. E.; ERNZERHOF, M. Hybrid functionals based on a screened coulomb potential. Journal of Chemical Physics, v. 118, n. 18, p. 8207-8215, 2003.

33 PERDEW, J. P.; ERNZERHOF, M.; BURKE, K. Rationale for mixing exact exchange with density functional approximations. Journal of Chemical Physics, v. 105, n. 22, p. 9982-9985, 1996.

34 KRUKAU, A. V. et al. Influence of the exchange screening parameter on the performance of screened hybrid functionals. Journal of Chemical Physics, v. 125, n. 22, p. 224106, 2006.

35 BUC̆KO, T. et al. Improved description of the structure of molecular and layered crystals: Ab Initio DFT calculations with van der Waals corrections. Journal of Physical Chemistry A, v. 114, n. 43, p. 11814-11824, 2010.

36 TKATCHENKO, A.; SCHEFFLER, M. Accurate molecular van der Waals interactions from ground-state electron density and free-atom reference data. Physical Review Letters, v. 102, n. 7, p. 073005, 2009.

37 TKATCHENKO, A. et al. Accurate and efficient method for many-body van der Waals interactions. Physical Review Letters, v. 108, n. 23, p. 236402, 2012.

38 GRIMME, S. et al. A consistent and accurate Ab Initio parametrization of density functional dispersion correction (DFT-D) for the 94 elements H-Pu. Journal of Chemical Physics, v. 132, n. 15, p. 154104, 2010.

39 RECKIEN, W. et al. Implementation of empirical dispersion corrections to density functional theory for periodic systems. Journal of Computational Chemistry, v. 33, n. 25, p. 2023-2031, 2012.

40 KITTEL, C. Introduction to solid state physics. 8th ed. New York: John Wiley \& Sons, 2004.

41 BLÖCHL, P. E. Projector augmented-wave method. Physical Review B, v. 50, n. 24, p. 17953-17979, 1994.

42 FEYNMAN, R. P. Forces in molecules. Physical Review, v. 56, n. 4, p. 340-343, 1939 .

43 KRESSE, G.; JOUBERT, D. From ultrasoft pseudopotentials to the projector augmented-wave method. Physical Review B, v. 59, n. 3, p. 1758-1775, 1999.

44 NIELSEN, O. H.; MARTIN, R. M. First-principles calculation of stress. Physical Review Letters, v. 50, n. 9, p. 697-700, 1983.

45 BESSE, R.; SABINO, F. P.; DA SILVA, J. L. F. Electronic structure of layered quaternary chalcogenide materials for band-gap engineering: the example of $\mathrm{Cs}_{2} \mathrm{M}^{\mathrm{II}} \mathrm{M}_{3}^{\mathrm{IV}} \mathrm{Q}_{8}$. Physical Review B, v. 93, n. 16, p. 165205, 2016. 
46 BUC̆KO, T. et al. Tkatchenko-Scheffler van der Waals correction method with and without self-consistent screening applied to solids. Physical Review B, v. 87, n. 6, p. 064110, 2013.

47 HOPPE, R. Effective coordination numbers (ECoN) and mean active fictive ionic radii (MEFIR). Zeitschrift für Kristallographie, v. 150, n. 1-4, p. 23-52, 1979.

48 DA SILVA, J. L. F. Effective coordination concept applied for phase change $(\mathrm{GeTe})_{m}\left(\mathrm{Sb}_{2} \mathrm{Te}_{3}\right)_{n}$ compounds. Journal of Applied Physics, v. 109, n. 2, p. 023502 , 2011.

49 BADER, R. F. W. Molecular fragments or chemical bonds. Accounts of Chemical Research, v. 8, n. 1, p. 34-40, 1975.

50 BADER, R. F. W. Atoms in molecules: a quantum theory. Oxford: Clarendom Press, 1994.

51 PAULING, L. The nature of the chemical bond. Ithaca: Cornell University Press, 1960.

52 BJÖRKMAN, T. et al. van der Waals bonding in layered compounds from advanced density-functional first-principles calculations. Physical Review Letters, v. 108, n. 23, p. $235502,2012$.

53 ZACHARIA, R.; ULBRICHT, H.; HERTEL, T. Interlayer cohesive energy of graphite from thermal desorption of polyaromatic hydrocarbons. Physical Review B, v. 69, n. 15 , p. 155406, 2004.

54 LIU, Z. et al. Interlayer binding energy of graphite: a mesoscopic determination from deformation. Physical Review B, v. 85, n. 20, p. 205418, 2012.

55 ASHCROFT, N. W.; MERMIM, N. M. Solid state physics. New York: Holt-Saunders International Editions, 1976.

56 LIU, H.-R. et al. First-principles study on the effective masses of zinc-blend-derived $\mathrm{Cu}_{2} \mathrm{Zn}-\mathrm{IV}-\mathrm{VI}_{4}(\mathrm{IV}=\mathrm{Sn}, \mathrm{Ge}, \mathrm{Si}$ and $\mathrm{VI}=\mathrm{S}, \mathrm{Se})$. Journal of Applied Physics, v. 112, n. 9, p. 093717, 2012.

57 SUN, Y.-Y. et al. Chalcogenide perovskites for photovoltaics. Nano Letters, v. 15, n. 1, p. 581-585, 2015.

58 LI, Y.-H.; GONG, X. G.; WEI, S.-H. Ab Initio all-electron calculation of absolute volume deformation potentials of IV-IV, III-V, and II-VI semiconductors: the chemical trends. Physical Review B, v. 73, n. 24, p. 245206, 2006.

59 SEGEV, D.; WEI, S.-H. Structure-derived electronic and optical properties of transparent conducting oxides. Physical Review B, v. 71, n. 12, p. 125129, 2005.

60 YU, P. Y.; CARDONA, M. Fundamentals of semiconductors: physics and materials properties. 4th ed. Berlin: Springer-Verlag, 2010.

61 LEWIS, B. G.; PAINE, D. C. Applications and processing of transparent conducting oxides. MRS Bulletin, v. 25, n. 08, p. 22-27, 2000. 
62 SABINO, F. P. et al. Origin of and tuning the optical and fundamental band gaps in transparent conducting oxides: the case of $\mathrm{M}_{2} \mathrm{O}_{3}(\mathrm{M}=\mathrm{Al}, \mathrm{Ga}, \mathrm{In})$. Physical Review B, v. 92, n. 20, p. 205308, 2015.

63 WALSH, A. et al. Nature of the band gap of $\operatorname{In}_{2} \mathrm{O}_{3}$ revealed by first-principles calculations and X-ray spectroscopy. Physical Review Letters, v. 100, n. 16, p. 167402, 2008 .

64 GAJDOŠ, M. et al. Linear optical properties in the projector-augmented wave methodology. Physical Review B, v. 73, n. 4, p. 045112, 2006.

65 BESSE, R.; DA SILVA, J. L. F. The role of the alkali and chalcogen atoms on the stability of the layered chalcogenide $\mathrm{A}_{2} \mathrm{M}^{\mathrm{II}} \mathrm{M}_{3}^{\mathrm{IV}} \mathrm{Q}_{8}$ (A=alkali-metal; $\mathrm{M}=$ metal-cations; $\mathrm{Q}=$ chalcogen) compounds: a density functional theory investigation within van der Waals corrections. Journal of Physics: condensed matter, v. 29, n. 3, p. 035402, 2017.

66 HWANG, S.-J.; IYER, R. G.; KANATZIDIS, M. G. Quaternary selenostannates $\mathrm{Na}_{2 x} \mathrm{Ga}_{2 x} \mathrm{Sn}_{1+x} \mathrm{Se}_{6}$ and $\mathrm{AGaSnSe}_{4}(\mathrm{~A}=\mathrm{K}, \mathrm{Rb}$, and $\mathrm{Cs}$ ) through rapid cooling of melts. kinetics versus thermodynamics in the polymorphism of $\mathrm{AGaSnSe}_{4}$. Journal of Solid State Chemistry, v. 177, n. 10, p. 3640-3649, 2004.

67 KANATZIDIS, M. G. Counterion size versus structure in metal-chalcogenide salts. Phosphorus, Sulfur, and Silicon and the Related Elements, v. 93, n. 1-4, p. 159-172, 1994. 



\section{Apêndices}





\section{APÊNDICE A - RESULTADOS SUPLEMENTARES DAS PROPRIEDADES ES- TRUTURAIS, ELETRÔNICAS E ÓPTICAS DOS CALCOGENE- TOS QUATERNÁRIOS $\mathrm{Cs}_{2} \mathbf{M}^{\mathrm{II}} \mathrm{M}_{3}^{\mathrm{IV}} \mathbf{Q}_{8}$}

Esse apêndice contém gráficos e tabelas que suplementam as discussões do Capítulo 3. Estão incluídos gráficos de estrutura de bandas e densidade de estados dos materiais não mostrados no Capítulo 3, além de duas tabelas, uma de PBE e outra de PBE+TS+SCS, com os resultados da análise do número de coordenação efetiva e distâncias médias de ligação de todos os compostos.

Tabela 7 - Resultados de números de coordenação efetiva, NCE, e distâncias médias de ligação, $\mathrm{d}_{\text {med }}$, dos 24 compostos $\mathrm{Cs}_{2} \mathrm{M}^{\mathrm{II}} \mathrm{M}_{3}^{\mathrm{IV}} \mathrm{Q}_{8}\left(\mathrm{M}^{\mathrm{II}}=\mathrm{Mg}, \mathrm{Zn}, \mathrm{Cd}, \mathrm{Hg} ; \mathrm{M}^{\mathrm{IV}}=\mathrm{Ge}, \mathrm{Sn} ; \mathrm{Q}=\right.$ $\mathrm{S}, \mathrm{Se}, \mathrm{Te})$ na estrutura ortorrômbica otimizada com PBE.

\begin{tabular}{|c|c|c|c|c|c|c|c|c|}
\hline \multirow[b]{2}{*}{ Composto } & \multicolumn{4}{|c|}{ NCE } & \multicolumn{4}{|c|}{$\mathrm{d}_{\text {med }}(\AA)$} \\
\hline & $\mathrm{Cs}$ & $\mathrm{M}^{\mathrm{II}}$ & $\mathrm{M}^{\mathrm{IV}}$ & Q & Cs & $\mathrm{M}^{\mathrm{II}}$ & $\mathrm{M}^{\mathrm{IV}}$ & $\mathrm{Q}$ \\
\hline $\mathrm{Cs}_{2} \mathrm{MgGe}_{3} \mathrm{~S}_{8}$ & 12,43 & 4,00 & 3,94 & 1,97 & 3,94 & 2,47 & 2,25 & 2,28 \\
\hline $\mathrm{Cs}_{2} \mathrm{MgGe}_{3} \mathrm{Se}_{8}$ & 12,71 & 4,00 & 3,97 & 1,90 & 4,06 & 2,61 & 2,40 & 2,43 \\
\hline $\mathrm{Cs}_{2} \mathrm{MgGe}_{3} \mathrm{Te}_{8}$ & 12,93 & 4,00 & 4,01 & 1,93 & 4,28 & 2,84 & 2,63 & 2,67 \\
\hline $\mathrm{Cs}_{2} \mathrm{MgSn}_{3} \mathrm{~S}_{8}{ }^{\circ}$ & 11,69 & 4,00 & 3,96 & 1,98 & 4,01 & 2,47 & 2,43 & 2,44 \\
\hline $\mathrm{Cs}_{2} \mathrm{MgSn}_{3} \mathrm{Se}_{8}$ & 12,46 & 4,00 & 3,97 & 1,99 & 4,15 & 2,61 & 2,57 & 2,58 \\
\hline $\mathrm{Cs}_{2} \mathrm{MgSn}_{3} \mathrm{Te}_{8}$ & 12,53 & 4,00 & 4,02 & 2,00 & 4,36 & 2,85 & 2,80 & 2,81 \\
\hline $\mathrm{Cs}_{2} \mathrm{ZnGe}_{3} \mathrm{~S}_{8}{ }^{\circ}$ & 12,72 & 4,00 & 3,95 & 1,94 & 3,93 & 2,37 & 2,25 & 2,27 \\
\hline $\mathrm{Cs}_{2} \mathrm{ZnGe}_{3} \mathrm{Se}_{8}$ & 13,09 & 4,00 & 3,97 & 1,96 & 4,05 & 2,51 & 2,40 & 2,42 \\
\hline $\mathrm{Cs}_{2} \mathrm{ZnGe}_{3} \mathrm{Te}_{8}$ & 13,44 & 3,99 & 4,02 & 1,99 & 4,25 & 2,71 & 2,63 & 2,65 \\
\hline $\mathrm{Cs}_{2} \mathrm{ZnSn}_{3} \mathrm{~S}_{8}$ & 12,26 & 4,00 & 3,96 & 2,00 & 4,00 & 2,38 & 2,43 & 2,42 \\
\hline $\mathrm{Cs}_{2} \mathrm{ZnSn}_{3} \mathrm{Se}_{8}$ & 12,85 & 4,00 & 3,97 & 2,00 & 4,13 & 2,50 & 2,57 & 2,56 \\
\hline $\mathrm{Cs}_{2} \mathrm{ZnSn}_{3} \mathrm{Te}_{8}$ & 12,13 & 3,99 & 4,01 & 2,00 & 4,28 & 2,70 & 2,78 & 2,78 \\
\hline $\mathrm{Cs}_{2} \mathrm{CdGe}_{3} \mathrm{~S}_{8}$ & 12,05 & 4,00 & 3,95 & 1,78 & 3,93 & 2,56 & 2,25 & 2,28 \\
\hline $\mathrm{Cs}_{2} \mathrm{CdGe}_{3} \mathrm{Se}_{8}$ & 12,51 & 4,01 & 3,97 & 1,84 & 4,06 & 2,69 & 2,40 & 2,43 \\
\hline $\mathrm{Cs}_{2} \mathrm{CdGe}_{3} \mathrm{Te}_{8}$ & 13,06 & 4,00 & 4,02 & 1,90 & 4,28 & 2,88 & 2,63 & 2,67 \\
\hline $\mathrm{Cs}_{2} \mathrm{CdSn}_{3} \mathrm{~S}_{8}$ & 11,73 & 4,00 & 3,96 & 1,94 & 4,01 & 2,57 & 2,43 & 2,45 \\
\hline $\mathrm{Cs}_{2} \mathrm{CdSn}_{3} \mathrm{Se}_{8}$ & 12,20 & 4,00 & 3,98 & 1,96 & 4,15 & 2,70 & 2,57 & 2,59 \\
\hline $\mathrm{Cs}_{2} \mathrm{CdSn}_{3} \mathrm{Te}_{8}$ & 12,64 & 4,00 & 4,02 & 1,99 & 4,36 & 2,89 & 2,80 & 2,82 \\
\hline $\mathrm{Cs}_{2} \mathrm{HgGe}_{3} \mathrm{~S}_{8}{ }^{\circ}$ & 12,07 & 4,00 & 3,95 & 1,75 & 3,93 & 2,59 & 2,25 & 2,28 \\
\hline $\mathrm{Cs}_{2} \mathrm{HgGe}_{3} \mathrm{Se}_{8}$ & 12,53 & 4,01 & 3,97 & 1,81 & 4,05 & 2,71 & 2,40 & 2,43 \\
\hline $\mathrm{Cs}_{2} \mathrm{HgGe}_{3} \mathrm{Te}_{8}$ & 12,88 & 4,00 & 4,02 & 1,90 & 4,26 & 2,90 & 2,64 & 2,67 \\
\hline $\mathrm{Cs}_{2} \mathrm{HgSn}_{3} \mathrm{~S}_{8}$ & 11,44 & 3,99 & 3,96 & 1,93 & 4,00 & 2,60 & 2,43 & 2,46 \\
\hline $\mathrm{Cs}_{2} \mathrm{HgSn}_{3} \mathrm{Se}_{8}$ & 12,18 & 4,00 & 3,98 & 1,95 & 4,14 & 2,73 & 2,57 & 2,57 \\
\hline $\mathrm{Cs}_{2} \mathrm{HgSn}_{3} \mathrm{Te}_{8}$ & 12,51 & 4,00 & 4,02 & 1,99 & 4,34 & 2,90 & 2,80 & 2,83 \\
\hline
\end{tabular}

Fonte: BESSE; SABINO; DA SILVA. ${ }^{45}$ 
Tabela 8 - Resultados de números de coordenação efetiva, NCE, e distâncias médias de ligação, $\mathrm{d}_{\text {med }}$, dos 24 compostos $\mathrm{Cs}_{2} \mathrm{M}^{\mathrm{II}} \mathrm{M}_{3}^{\mathrm{IV}} \mathrm{Q}_{8}\left(\mathrm{M}^{\mathrm{II}}=\mathrm{Mg}\right.$, Zn, Cd, Hg; $\mathrm{M}^{\mathrm{IV}}=\mathrm{Ge}, \mathrm{Sn} ; \mathrm{Q}=$ $\mathrm{S}, \mathrm{Se}, \mathrm{Te}$ ) na estrutura ortorrômbica otimizada com PBE+TS+SCS.

\begin{tabular}{|c|c|c|c|c|c|c|c|c|}
\hline \multirow[b]{2}{*}{ Composto } & \multicolumn{4}{|c|}{$\mathrm{NCE}(\mathrm{NV})$} & \multicolumn{4}{|c|}{$\mathrm{d}_{\text {med }}(\AA)$} \\
\hline & Cs & $\mathrm{M}^{\mathrm{II}}$ & $\mathrm{M}^{\mathrm{IV}}$ & Q & Cs & $\mathrm{M}^{\mathrm{II}}$ & $\mathrm{M}^{\mathrm{IV}}$ & Q \\
\hline $\mathrm{Cs}_{2} \mathrm{MgGe}_{3} \mathrm{~S}_{8}$ & 14,46 & 4,00 & 3,94 & 1,89 & 3,72 & 2,44 & 2,24 & 2,27 \\
\hline $\mathrm{Cs}_{2} \mathrm{MgGe}_{3} \mathrm{Se}_{8}$ & 14,19 & 4,00 & 3,96 & 1,93 & 3,82 & 2,58 & 2,40 & 2,43 \\
\hline $\mathrm{Cs}_{2} \mathrm{MgGe}_{3} \mathrm{Te}_{8}$ & 13,46 & 4,01 & 4,04 & 2,02 & 4,03 & 2,80 & 2,63 & 2,67 \\
\hline $\mathrm{Cs}_{2} \mathrm{MgSn}_{3} \mathrm{~S}_{8}$ & 14,56 & 4,00 & 3,95 & 2,00 & 3,83 & 2,44 & 2,43 & 2,43 \\
\hline $\mathrm{Cs}_{2} \mathrm{MgSn}_{3} \mathrm{Se}_{8}$ & 14,37 & 4,00 & 3,97 & 2,05 & 3,94 & 2,58 & 2,57 & 2,58 \\
\hline $\mathrm{Cs}_{2} \mathrm{MgSn}_{3} \mathrm{Te}_{8}$ & 12,25 & 4,00 & 4,05 & 2,22 & 4,04 & 2,79 & 2,82 & 2,84 \\
\hline $\mathrm{Cs}_{2} \mathrm{ZnGe}_{3} \mathrm{~S}_{8}$ & 14,66 & 4,00 & 3,94 & 1,95 & 3,69 & 2,35 & 2,24 & 2,27 \\
\hline $\mathrm{Cs}_{2} \mathrm{ZnGe}_{3} \mathrm{Se}_{8}$ & 14,33 & 4,00 & 3,97 & 1,98 & 3,79 & 2,48 & 2,40 & 2,41 \\
\hline $\mathrm{Cs}_{2} \mathrm{ZnGe}_{3} \mathrm{Te}_{8}$ & 13,21 & 3,99 & 4,03 & 2,04 & 3,97 & 2,66 & 2,64 & 2,65 \\
\hline $\mathrm{Cs}_{2} \mathrm{ZnSn}_{3} \mathrm{~S}_{8}$ & 14,56 & 4,00 & 3,96 & 2,02 & 3,79 & 2,35 & 2,43 & 2,41 \\
\hline $\mathrm{Cs}_{2} \mathrm{ZnSn}_{3} \mathrm{Se}_{8}$ & 14,07 & 4,00 & 3,97 & 2,05 & 3,89 & 2,48 & 2,57 & 2,56 \\
\hline $\mathrm{Cs}_{2} \mathrm{ZnSn}_{3} \mathrm{Te}_{8}$ & 12,35 & 3,99 & 4,05 & 2,17 & 4,02 & 2,67 & 2,81 & 2,80 \\
\hline $\mathrm{Cs}_{2} \mathrm{CdGe}_{3} \mathrm{~S}_{8}$ & 14,07 & 4,01 & 3,94 & 1,78 & 3,70 & 2,55 & 2,24 & 2,27 \\
\hline $\mathrm{Cs}_{2} \mathrm{CdGe}_{3} \mathrm{Se}_{8}$ & 14,03 & 4,02 & 3,97 & 1,87 & 3,81 & 2,67 & 2,40 & 2,43 \\
\hline $\mathrm{Cs}_{2} \mathrm{CdGe}_{3} \mathrm{Te}_{8}$ & 13,38 & 4,02 & 4,04 & 2,01 & 4,02 & 2,85 & 2,63 & 2,68 \\
\hline $\mathrm{Cs}_{2} \mathrm{CdSn}_{3} \mathrm{~S}_{8}$ & 14,44 & 4,00 & 3,95 & 1,98 & 3,82 & 2,55 & 2,42 & 2,45 \\
\hline $\mathrm{Cs}_{2} \mathrm{CdSn}_{3} \mathrm{Se}_{8}$ & 14,26 & 4,00 & 3,97 & 2,07 & 3,94 & 2,67 & 2,57 & 2,60 \\
\hline $\mathrm{Cs}_{2} \mathrm{CdSn}_{3} \mathrm{Te}_{8}$ & 12,20 & 4,01 & 4,06 & 2,27 & 4,05 & 2,85 & 2,81 & 2,86 \\
\hline $\mathrm{Cs}_{2} \mathrm{HgGe}_{3} \mathrm{~S}_{8}$ & 14,00 & 4,00 & 3,94 & 1,75 & 3,69 & 2,58 & 2,24 & 2,27 \\
\hline $\mathrm{Cs}_{2} \mathrm{HgGe}_{3} \mathrm{Se}_{8}$ & 14,00 & 4,03 & 3,97 & 1,85 & 3,81 & 2,70 & 2,40 & 2,43 \\
\hline $\mathrm{Cs}_{2} \mathrm{HgGe}_{3} \mathrm{Te}_{8}$ & 13,64 & 4,01 & 4,05 & 2,05 & 4,03 & 2,87 & 2,63 & 2,69 \\
\hline $\mathrm{Cs}_{2} \mathrm{HgSn}_{3} \mathrm{~S}_{8}$ & 14,33 & 3,99 & 3,95 & 1,98 & 3,81 & 2,59 & 2,43 & 2,46 \\
\hline $\mathrm{Cs}_{2} \mathrm{HgSn}_{3} \mathrm{Se}_{8}$ & 14,25 & 4,01 & 3,98 & 2,09 & 3,94 & 2,70 & 2,57 & 2,60 \\
\hline $\mathrm{Cs}_{2} \mathrm{HgSn}_{3} \mathrm{Te}_{8}$ & 12,33 & 3,99 & 4,07 & 2,34 & 4,05 & 2,86 & 2,82 & 2,87 \\
\hline
\end{tabular}

Fonte: BESSE; SABINO; DA SILVA. ${ }^{45}$ 

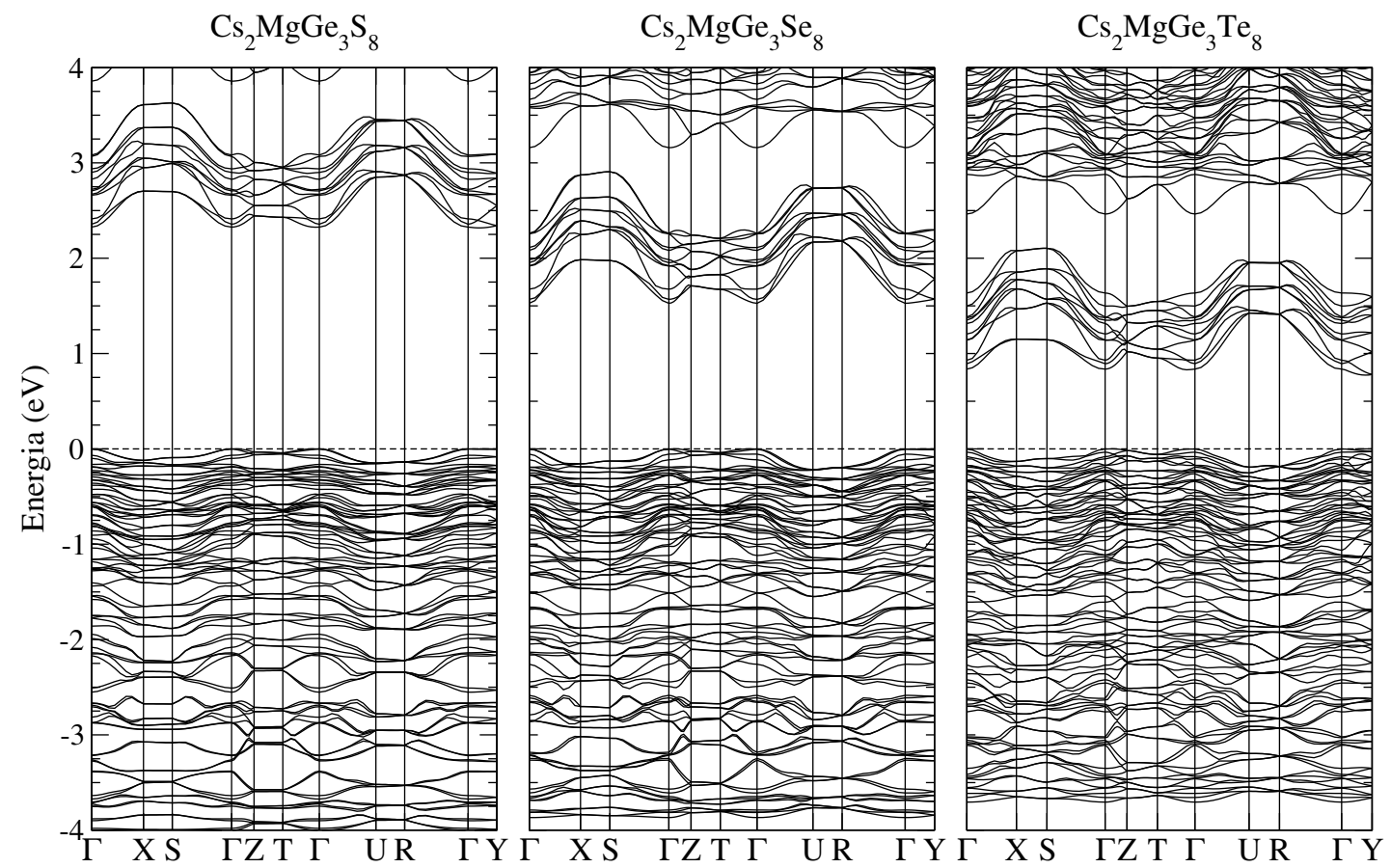

Figura 18 - Estruturas de bandas dos compostos $\mathrm{Cs}_{2} \mathrm{MgGe}_{3} \mathrm{Q}_{8}(\mathrm{Q}=\mathrm{S}$, Se, Te), calculadas com PBE. O topo da banda de valência está deslocado para energia zero.

Fonte: Adaptada de BESSE; SABINO; DA SILVA. ${ }^{45}$
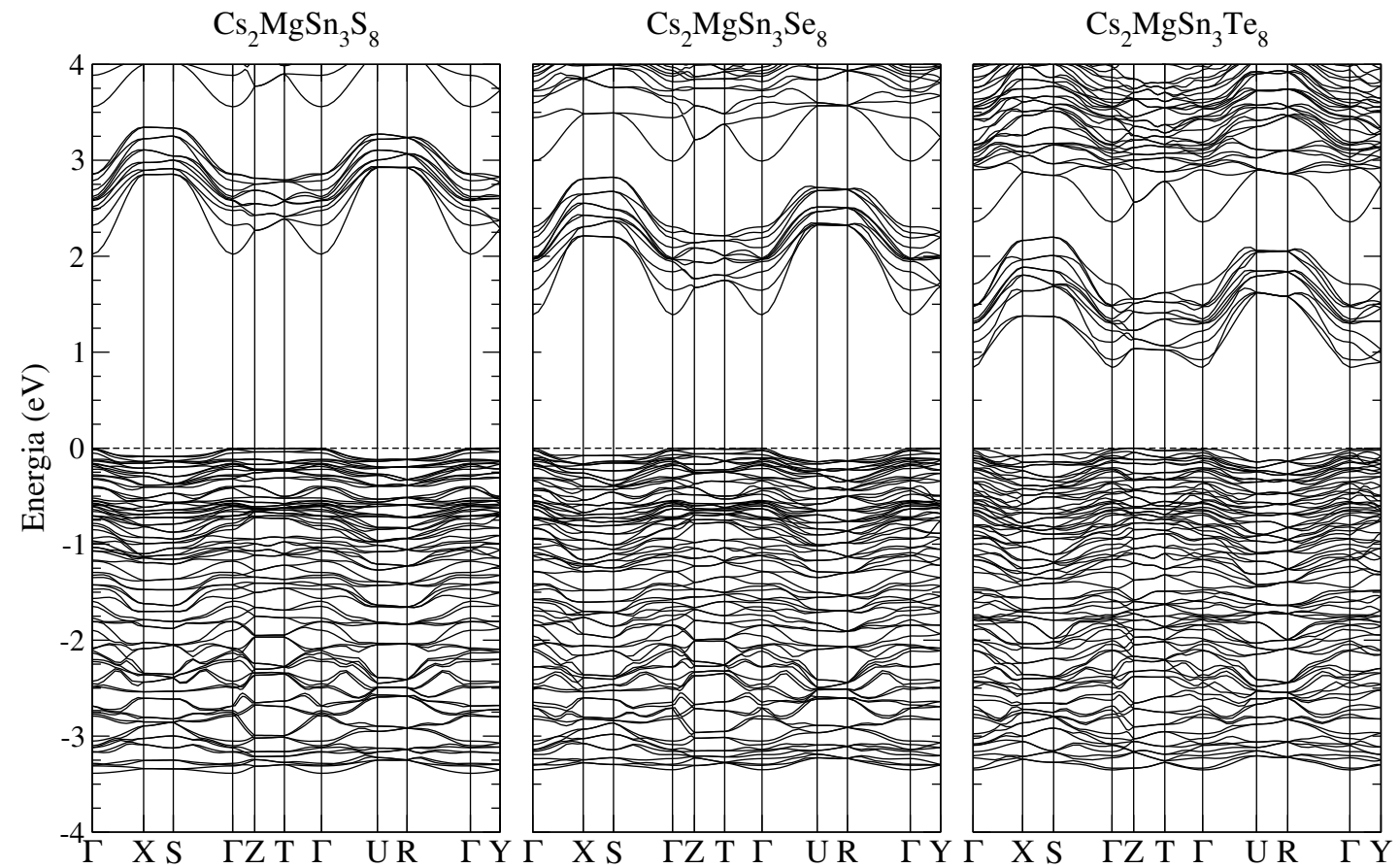

Figura 19 - Estruturas de bandas dos compostos $\mathrm{Cs}_{2} \mathrm{MgSn}_{3} \mathrm{Q}_{8}(\mathrm{Q}=\mathrm{S}, \mathrm{Se}, \mathrm{Te})$, calculadas com PBE. O topo da banda de valência está deslocado para energia zero.

Fonte: Adaptada de BESSE; SABINO; DA SILVA. ${ }^{45}$ 


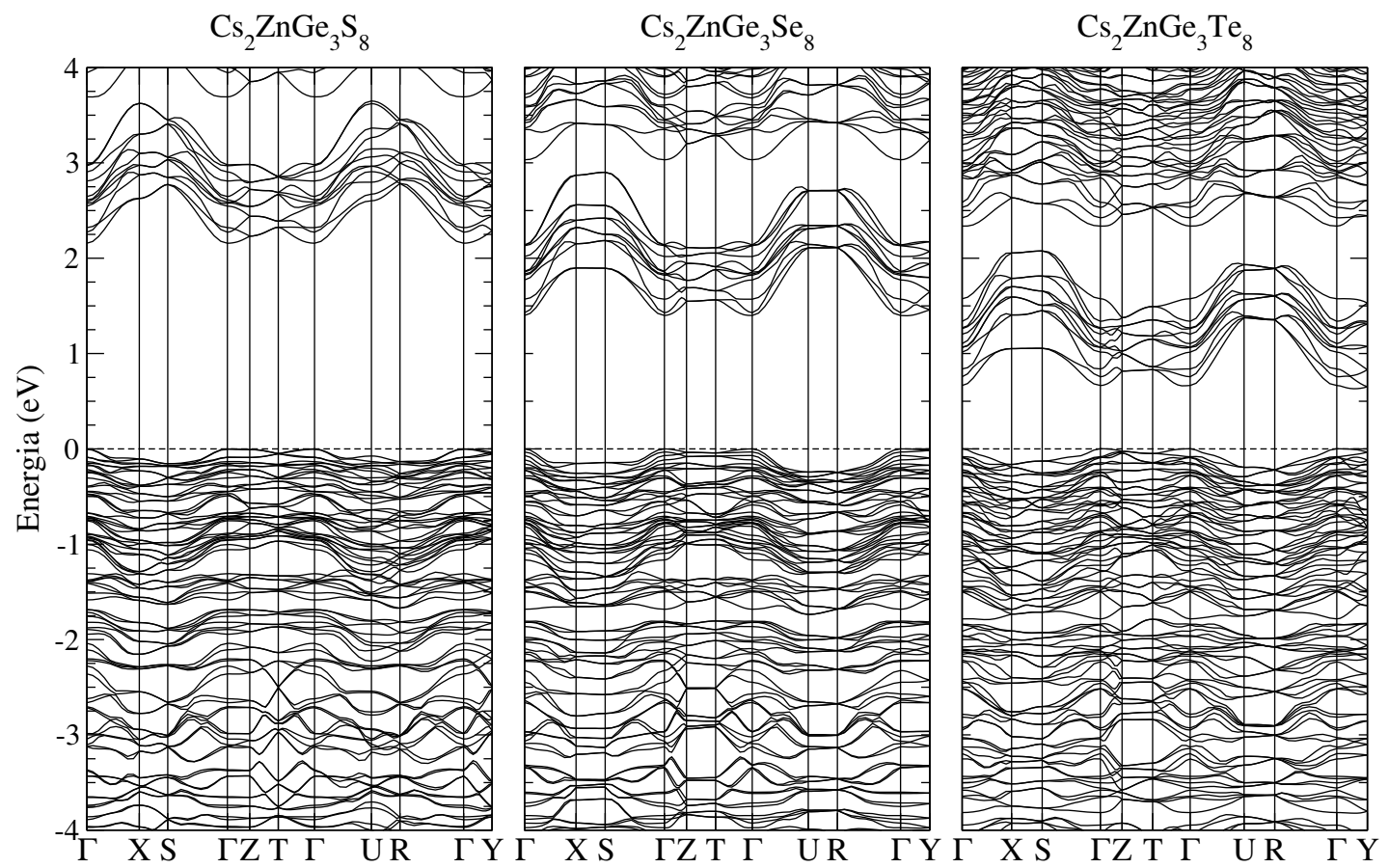

Figura 20 - Estruturas de bandas dos compostos $\mathrm{Cs}_{2} \mathrm{ZnGe}_{3} \mathrm{Q}_{8}(\mathrm{Q}=\mathrm{S}, \mathrm{Se}, \mathrm{Te})$, calculadas com PBE. O topo da banda de valência está deslocado para energia zero.

Fonte: Adaptada de BESSE; SABINO; DA SILVA. ${ }^{45}$
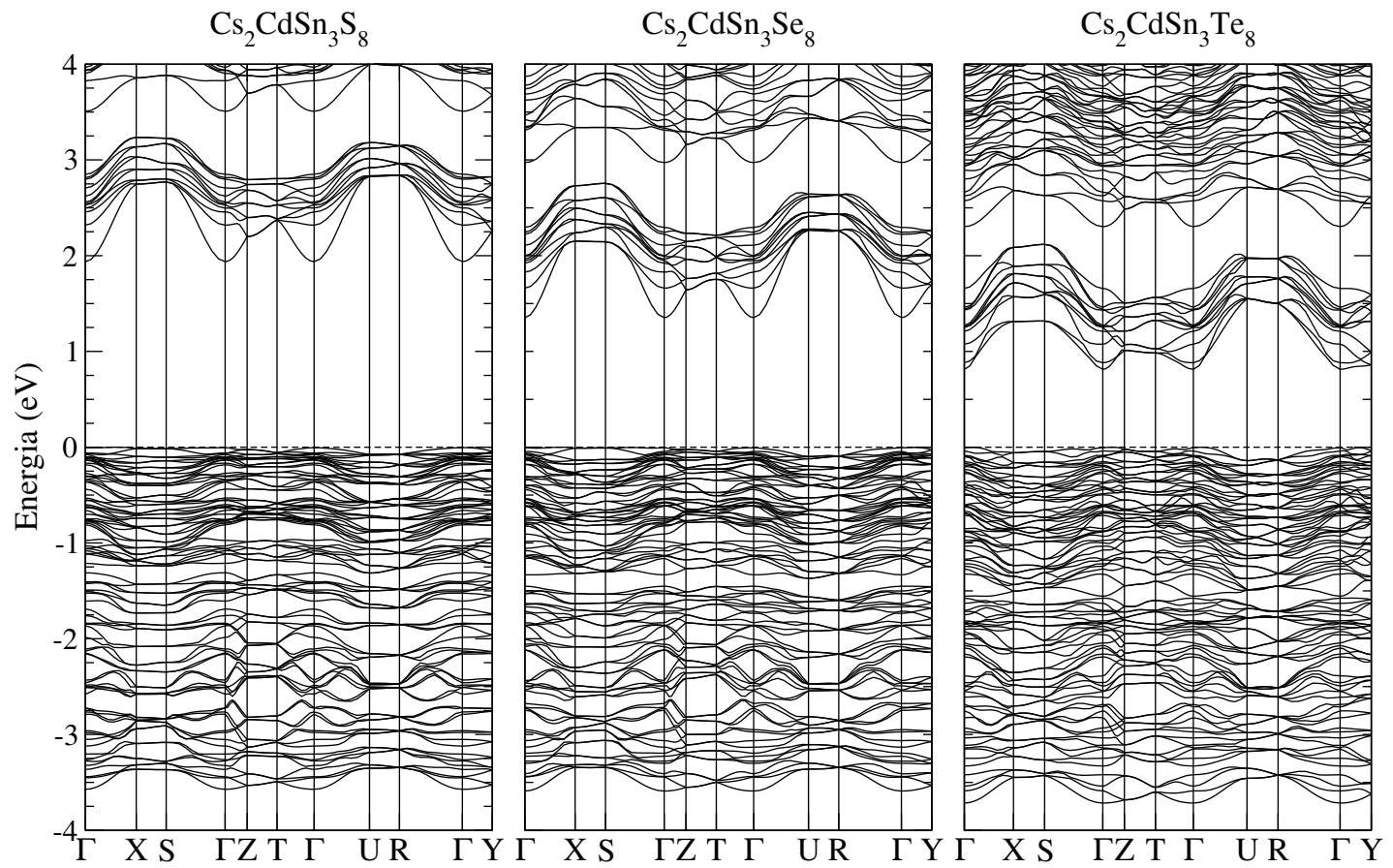

Figura 21 - Estruturas de bandas dos compostos $\mathrm{Cs}_{2} \mathrm{CdSn}_{3} \mathrm{Q}_{8}(\mathrm{Q}=\mathrm{S}, \mathrm{Se}, \mathrm{Te})$, calculadas com PBE. O topo da banda de valência está deslocado para energia zero.

Fonte: Adaptada de BESSE; SABINO; DA SILVA. ${ }^{45}$ 

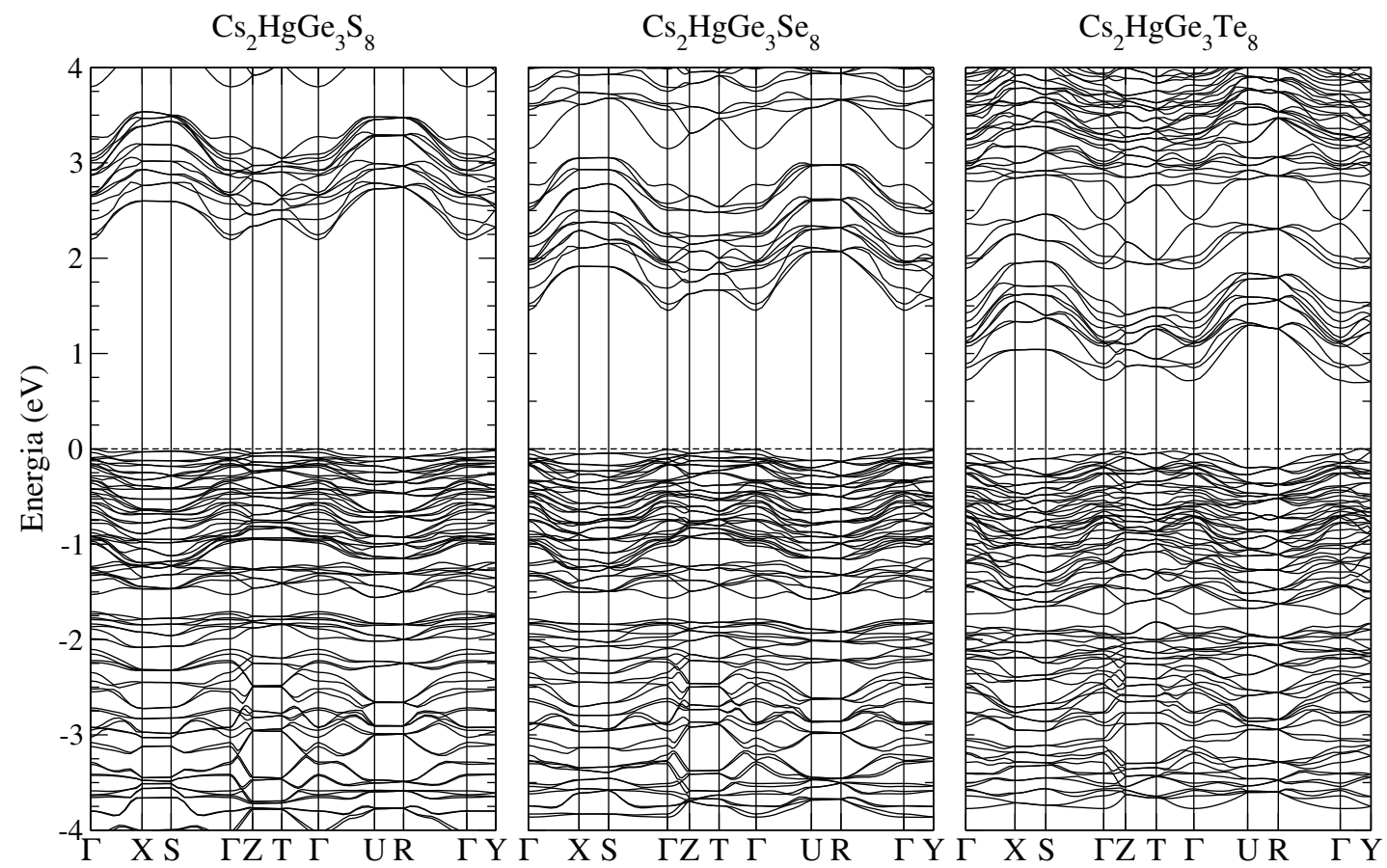

Figura 22 - Estruturas de bandas dos compostos $\mathrm{Cs}_{2} \mathrm{HgGe}_{3} \mathrm{Q}_{8}(\mathrm{Q}=\mathrm{S}, \mathrm{Se}, \mathrm{Te})$, calculadas com PBE. O topo da banda de valência está deslocado para energia zero.

Fonte: Adaptada de BESSE; SABINO; DA SILVA. ${ }^{45}$
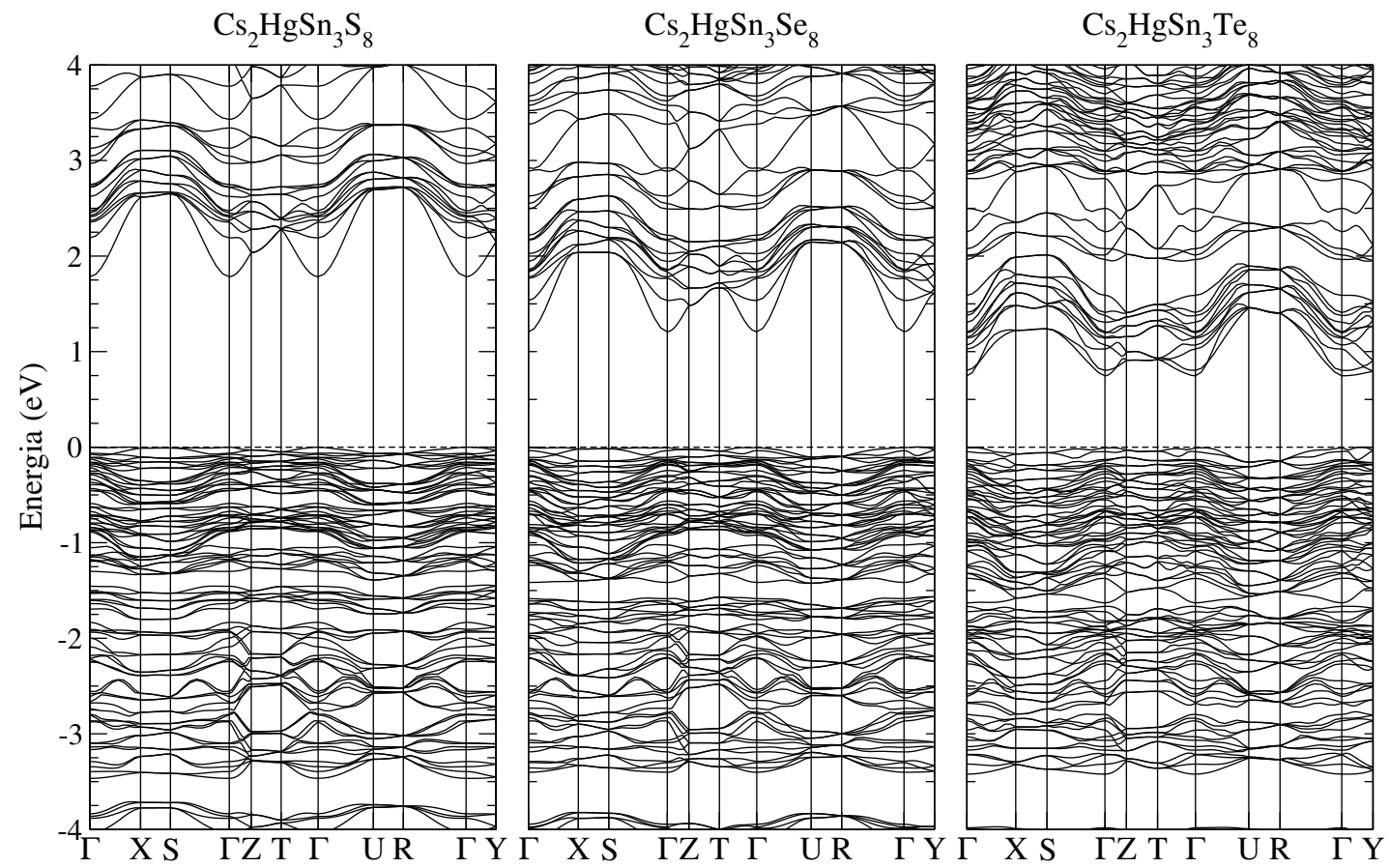

Figura 23 - Estruturas de bandas dos compostos $\mathrm{Cs}_{2} \mathrm{HgSn}_{3} \mathrm{Q}_{8}(\mathrm{Q}=\mathrm{S}, \mathrm{Se}, \mathrm{Te})$, calculadas com PBE. O topo da banda de valência está deslocado para energia zero.

Fonte: Adaptada de BESSE; SABINO; DA SILVA. ${ }^{45}$ 


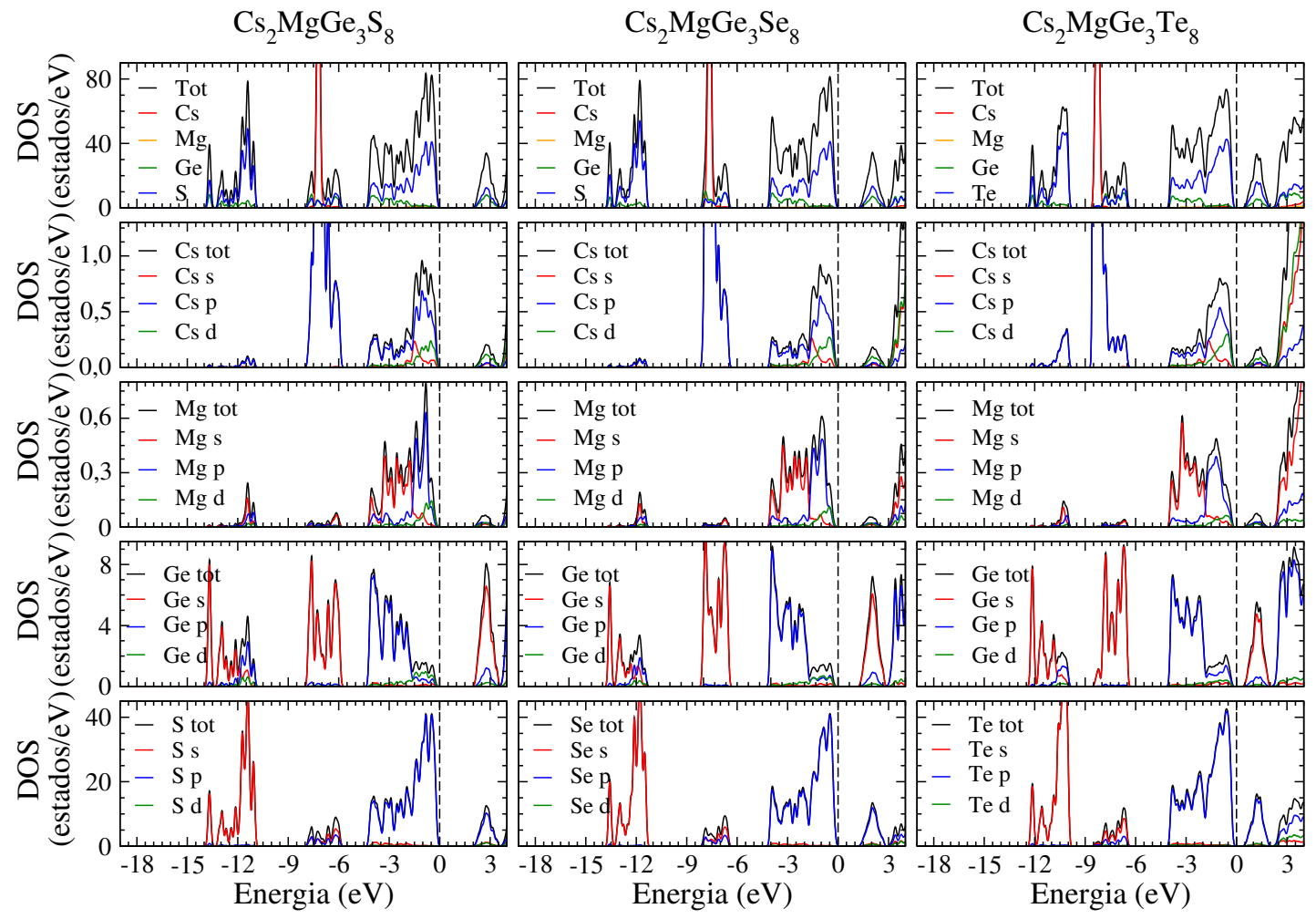

Figura 24 - Densidade de estados por célula cristalina dos compostos $\mathrm{Cs}_{2} \mathrm{MgGe}_{3} \mathrm{Q}_{8}(\mathrm{Q}=\mathrm{S}, \mathrm{Se}$, Te), calculadas com PBE. O topo da banda de valência está deslocado para energia zero.

Fonte: Adaptada de BESSE; SABINO; DA SILVA. ${ }^{45}$ 


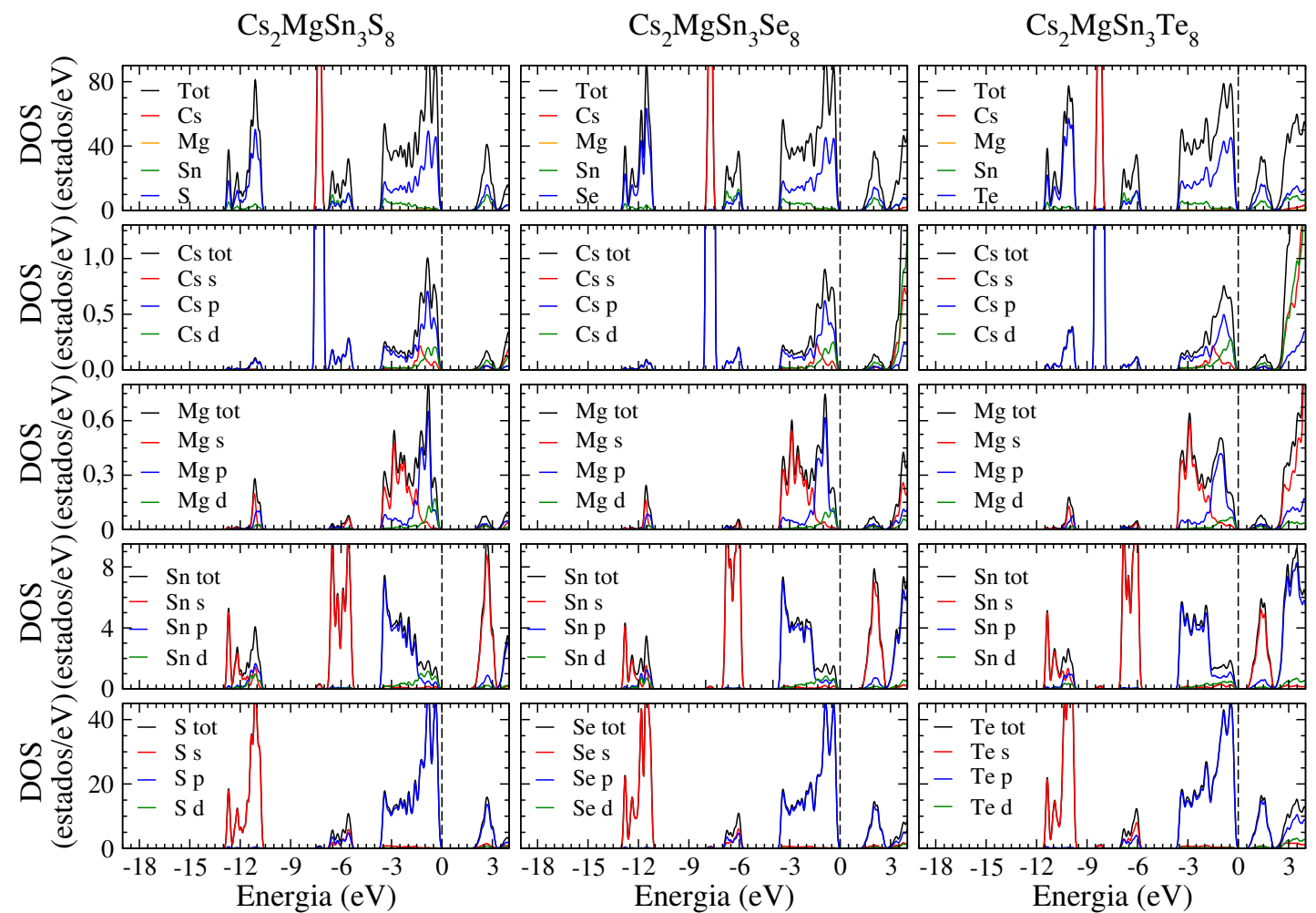

Figura 25 - Densidade de estados por célula cristalina dos compostos $\mathrm{Cs}_{2} \mathrm{MgSn}_{3} \mathrm{Q}_{8}(\mathrm{Q}=\mathrm{S}, \mathrm{Se}$, Te), calculadas com PBE. O topo da banda de valência está deslocado para energia zero.

Fonte: Adaptada de BESSE; SABINO; DA SILVA. ${ }^{45}$ 


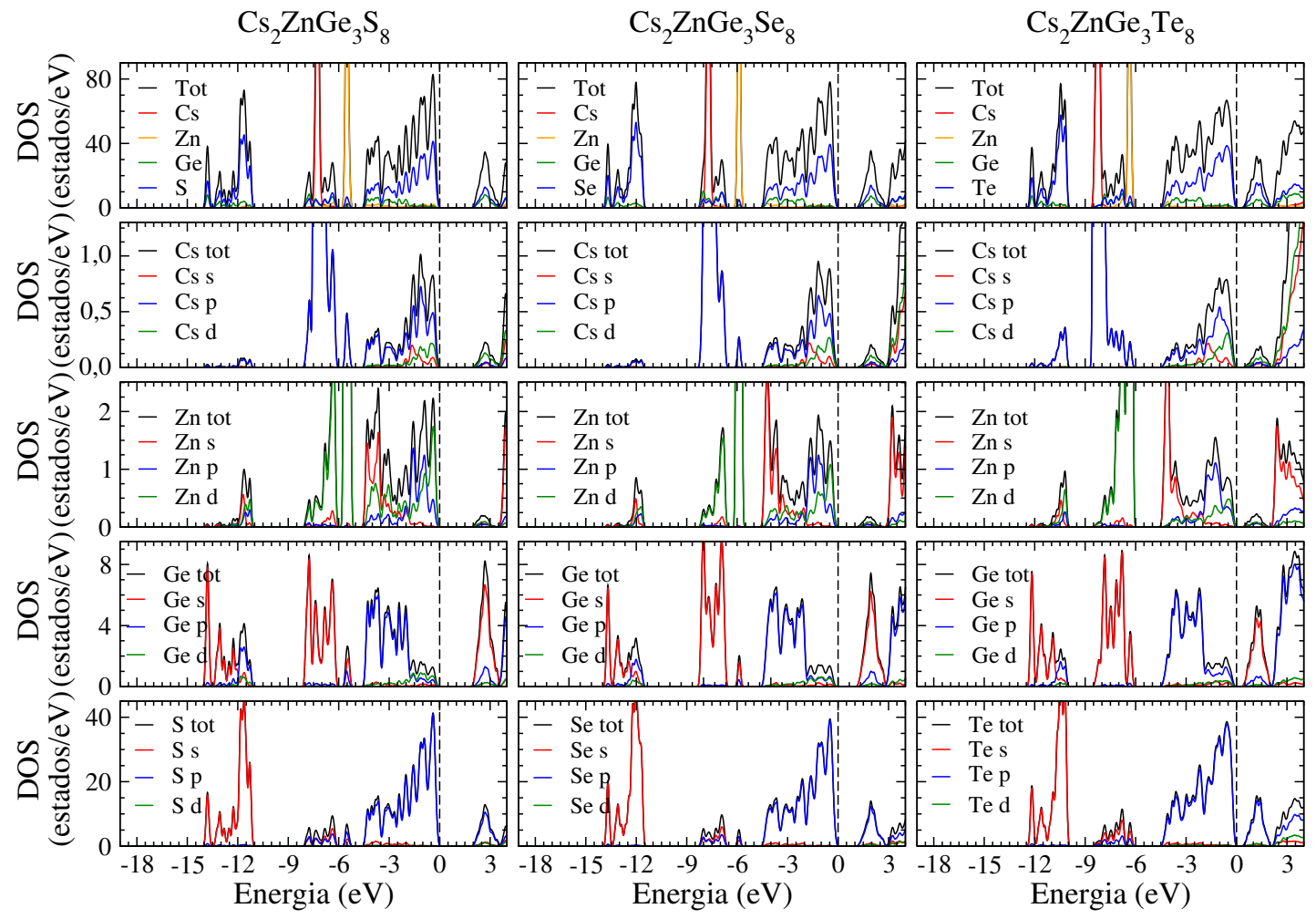

Figura 26 - Densidade de estados por célula cristalina dos compostos $\mathrm{Cs}_{2} \mathrm{ZnGe}_{3} \mathrm{Q}_{8}(\mathrm{Q}=\mathrm{S}, \mathrm{Se}$, Te), calculadas com PBE. O topo da banda de valência está deslocado para energia zero.

Fonte: Adaptada de BESSE; SABINO; DA SILVA. ${ }^{45}$ 


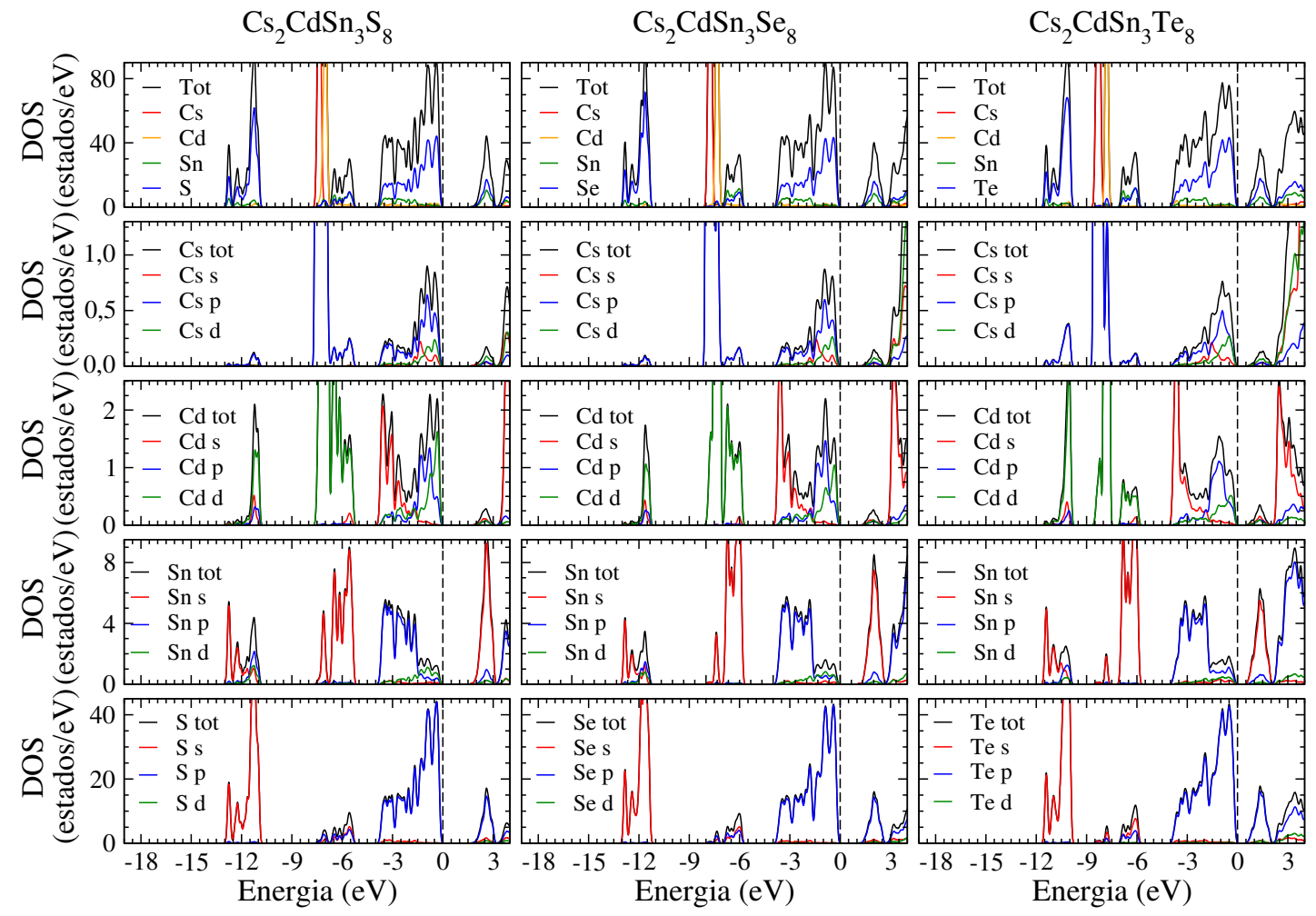

Figura 27 - Densidade de estados por célula cristalina dos compostos $\mathrm{Cs}_{2} \mathrm{CdSn}_{3} \mathrm{Q}_{8}(\mathrm{Q}=\mathrm{S}$, Se, $\mathrm{Te})$, calculadas com PBE. O topo da banda de valência está deslocado para energia zero.

Fonte: Adaptada de BESSE; SABINO; DA SILVA. ${ }^{45}$ 


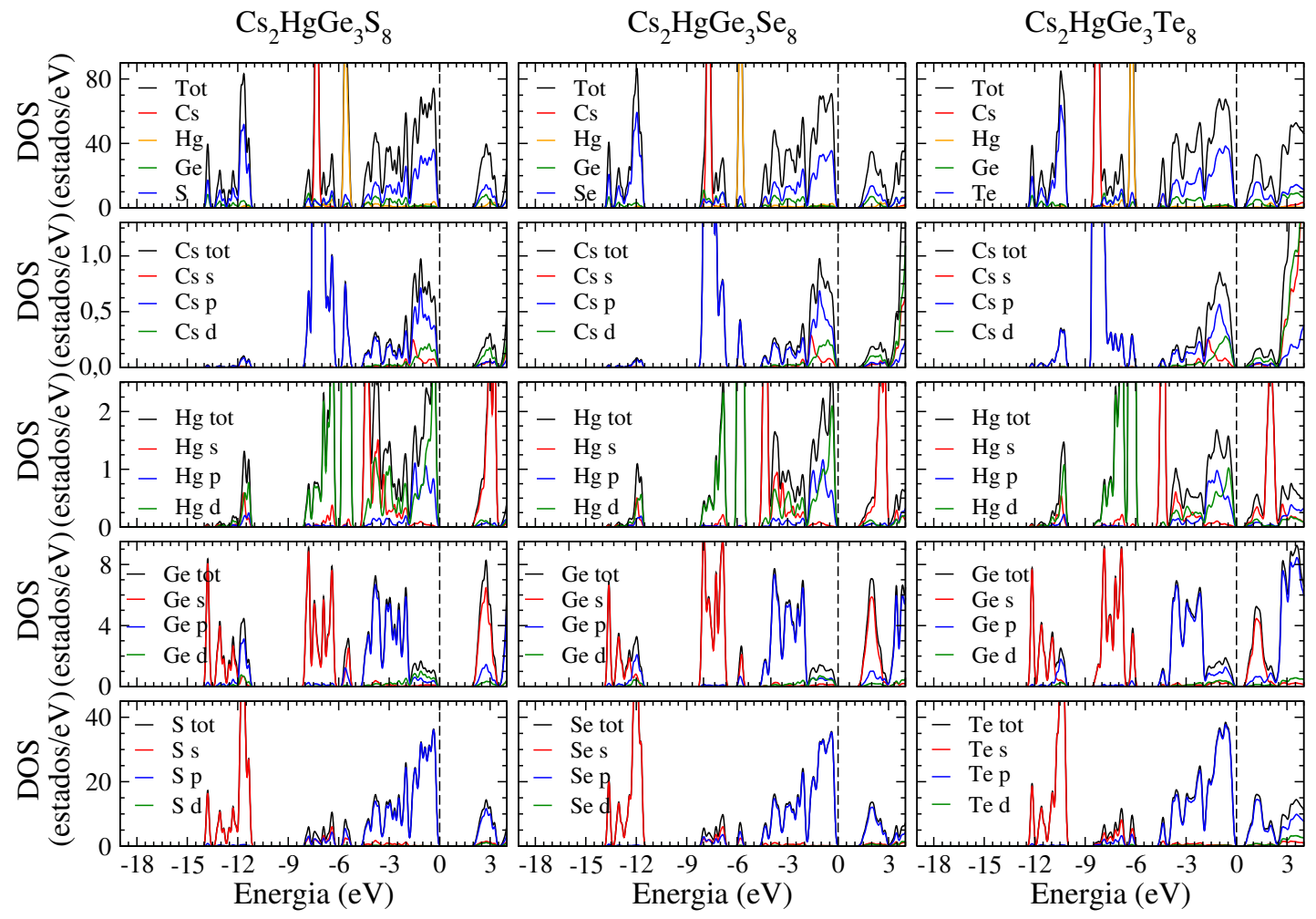

Figura 28 - Densidade de estados por célula cristalina dos compostos $\mathrm{Cs}_{2} \mathrm{HgGe}_{3} \mathrm{Q}_{8}(\mathrm{Q}=\mathrm{S}, \mathrm{Se}$, $\mathrm{Te}$ ), calculadas com PBE. O topo da banda de valência está deslocado para energia zero.

Fonte: Adaptada de BESSE; SABINO; DA SILVA. ${ }^{45}$ 


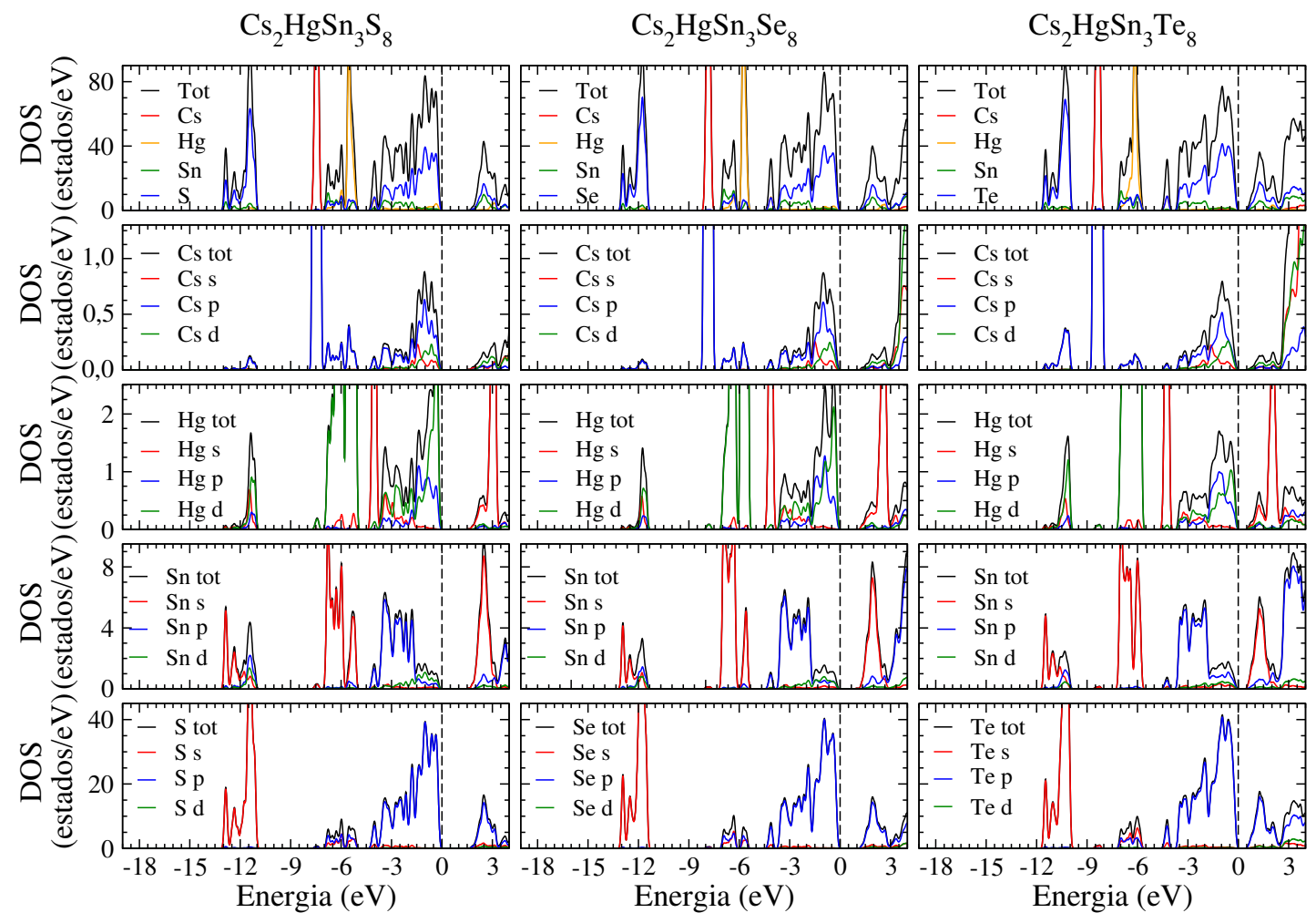

Figura 29 - Densidade de estados por célula cristalina dos compostos $\mathrm{Cs}_{2} \mathrm{HgSn}_{3} \mathrm{Q}_{8}(\mathrm{Q}=\mathrm{S}$, Se, Te), calculadas com PBE. O topo da banda de valência está deslocado para energia zero.

Fonte: Adaptada de BESSE; SABINO; DA SILVA. ${ }^{45}$ 